\title{
NONARCHIMEDEAN GEOMETRY OF WITT VECTORS
}

\author{
KIRAN S. KEDLAYA
}

\begin{abstract}
Let $R$ be a perfect $\mathbb{F}_{p}$-algebra equipped with the trivial norm. Let $W(R)$ be the ring of $p$-typical Witt vectors over $R$ equipped with the $p$-adic norm. At the level of nonarchimedean analytic spaces (in the sense of Berkovich), we demonstrate a close analogy between $W(R)$ and the polynomial ring $R[T]$ equipped with the Gauss norm, in which the role of the structure morphism from $R$ to $R[T]$ is played by the Teichmüller map. For instance, we show that the analytic space associated to $R$ is a strong deformation retract of the space associated to $W(R)$. We also show that each fiber forms a tree under the relation of pointwise comparison, and we classify the points of fibers in the manner of Berkovich's classification of points of a nonarchimedean disk. Some results pertain to the study of $p$-adic representations of étale fundamental groups of nonarchimedean analytic spaces (i.e., relative $p$-adic Hodge theory).
\end{abstract}

\section{Contents}

0. Introduction ... . . . . . . . . . . . . . . . . . 111

1. Nonarchimedean analytic spaces . . . . . . . . . . . . . . . . . . . . 113

2. Nonarchimedean geometry of polynomial rings . . . . . . . . . . . . . . . . 119

3. Witt vectors . . . . . . . . . . . . . . . . . . . . . 130

4. Raising and lowering for Witt vectors . . . . . . . . . . . . . . . . . . . 134

5. Gauss norms . . . . . . . . . . . . . . . . . . . . . . 139

6. Newton polygons and factorizations . . . . . . . . . . . . . . . . . . . . 144

7. Restriction of Gauss norms . . . . . . . . . . . . . . . . . . . . . . . . 148

8. Structure of fibers . . . . . . . . . . . . . . . . . . . . . . . . 154

References ............................ 163

\section{$\S 0$. Introduction}

There now exist several approaches to nonarchimedean analytic geometry, including rigid analytic geometry (Tate), formal geometry (Raynaud), and

Received July 29, 2010. Revised February 9, 2012. Accepted February 15, 2012.

2010 Mathematics Subject Classification. Primary 14G22; Secondary 13 F35.

The author was partially supported by National Science Foundation grants DMS0545904 and DMS-0635607, by DARPA grant HR0011-09-1-0048, by the NEC fund and the Cecil and Ida Green professorship at the Massachusetts Institute of Technology, and by the James D. Wolfensohn Fund of the Institute for Advanced Study.

(C) 2013 by The Editorial Board of the Nagoya Mathematical Journal 
adic geometry (Huber, Fujiwara-Kato). However, the approach exhibiting the closest links with classical topology is that of Berkovich [2]. Berkovich overcomes the lack of connectivity of nonarchimedean topologies by considering spaces of multiplicative seminorms, using an analogue of the usual Gel'fand transform relating commutative Banach algebras to spaces of continuous functions on compact topological spaces.

Much is known about the topology of Berkovich analytic spaces. For instance, Berkovich showed that smooth analytic spaces over a field are locally contractible (see [4], [5]). More recently, Payne [24] showed that the analytification of an algebraic variety over a field can be viewed as an inverse limit of finite polyhedral complexes; separately, Hrushovski and Loeser [15] have used model-theoretic techniques to show that such analytifications are locally contractible and retract onto finite $\mathrm{CW}$-complexes. One can also relate homotopy types of analytic spaces to degenerations; for instance, the analytification of a semistable curve over a complete discretely valued field has the same homotopy type as the graph of the special fiber of a minimal proper regular model over the valuation subring. This relationship, and its link to mixed Hodge structures, has been further pursued by Berkovich [6] and Nicaise [23].

In this paper, we consider degenerations in mixed characteristic from the point of view of Witt vectors. Recall that for $p$ a fixed prime number, the $p$-typical Witt vector functor converts perfect $\mathbb{F}_{p}$-algebras into $p$-adically complete algebras. Let $R$ be a perfect $\mathbb{F}_{p}$-algebra equipped with the trivial norm, and equip the associated Witt vector ring $W(R)$ with the $p$-adic norm. Let $\mathcal{M}(R)$ and $\mathcal{M}(W(R))$ denote the resulting Berkovich spaces. There is a natural multiplicative map $R \rightarrow W(R)$ given by Teichmüller lifting; this map is not a ring homomorphism, but it nonetheless induces a restriction map $\mu: \mathcal{M}(W(R)) \rightarrow \mathcal{M}(R)$ as if it were a homomorphism.

We establish several results that liken the relationship between $\mathcal{M}(R)$ and $\mathcal{M}(W(R))$ to the relationship between $\mathcal{M}(R)$ and $\mathcal{M}(R[T])$ when $R[T]$ carries the Gauss norm (i.e., between a base space and a disk bundle over the base). We first construct a continuous section $\lambda: \mathcal{M}(R) \rightarrow \mathcal{M}(W(R))$ giving a maximal lifting of a seminorm on $R$ to $W(R)$; this identifies $\mathcal{M}(R)$ as a retract of $\mathcal{M}(W(R))$. We then refine this calculation to show (Theorem 7.8) that $\mathcal{M}(R)$ is a strong deformation retract of $\mathcal{M}(W(R))$ and that any subset of $\mathcal{M}(R)$ has the same homotopy type as its inverse image under the projection $\mu$. We finally describe the geometry of the fibers of the projection map $\mu$ : each fiber may be naturally viewed as a tree in both a topological 
fashion (as an inverse limit of finite contractible 1-dimensional simplicial complexes) and a combinatorial fashion (as a partially ordered set in which any two elements dominating a common third element are comparable).

The motivation for this work is to describe $p$-adic Hodge theory (the study of continuous representations of Galois groups of finite extensions of the $p$-adic field $\mathbb{Q}_{p}$ ) in a fashion that permits consideration also of étale fundamental groups of analytic spaces. A preview of such a description is given in [20], together with an application to the construction of local systems on Rapoport-Zink period spaces; that preview already includes a few of the results given here, including the definition of the maps $\lambda$ and $\mu$. A related development is a reformulation of $p$-adic Hodge theory by Fargues and Fontaine [11], in which one works with coherent sheaves on an object constructed from a ring of Witt vectors, which behaves formally like an analytic curve.

\section{$\S 1$. Nonarchimedean analytic spaces}

We begin by setting notation and terminology concerning nonarchimedean analytic spaces, as in [2].

Definition 1.1. Consider the following conditions on an abelian group $G$ and a function $\alpha: G \rightarrow[0,+\infty)$.

(a) For all $g, h \in G$, we have $\alpha(g-h) \leq \max \{\alpha(g), \alpha(h)\}$.

(b) We have $\alpha(0)=0$.

$\left(\mathrm{b}^{\prime}\right)$ For all $g \in G$, we have $\alpha(g)=0$ if and only if $g=0$.

We say that $\alpha$ is a seminorm if it satisfies (a) and (b) and a norm if it satisfies (a) and $\left(\mathrm{b}^{\prime}\right)$. These would typically be called nonarchimedean seminorms and norms, but we will use no other kind in this paper.

If $\alpha, \alpha^{\prime}$ are two seminorms on the same abelian group $G$, we say that $\alpha$ dominates $\alpha^{\prime}$, and we write $\alpha \geq \alpha^{\prime}$ or $\alpha^{\prime} \leq \alpha$, if there exists $c \in(0,+\infty)$ for which $\alpha^{\prime}(g) \leq c \alpha(g)$ for all $g \in G$. If $\alpha$ and $\alpha^{\prime}$ dominate each other, we say that they are equivalent; in this case, $\alpha$ is a norm if and only if $\alpha^{\prime}$ is.

Let $G, H$ be two abelian groups equipped with seminorms $\alpha, \beta$. We say that a homomorphism $\phi: G \rightarrow H$ is bounded if $\alpha$ dominates $\beta \circ \phi$. We say that $\phi$ is isometric if $\alpha=\beta \circ \phi$.

Definition 1.2. Let $\alpha$ be a seminorm on an abelian group $G$. For any subgroup $H$ of $G, \alpha$ induces a quotient seminorm on $G / H$ defined by

$$
g+H \mapsto \inf \{\alpha(g+h): h \in H\}
$$


This defines a norm if $H$ is closed; for instance, the quotient seminorm on $G / \operatorname{ker}(\alpha)$ is a norm.

The group of Cauchy sequences in $G$ carries a seminorm defined by

$$
\left(x_{0}, x_{1}, \ldots\right) \mapsto \lim _{i \rightarrow \infty} \alpha\left(x_{i}\right) .
$$

Passing to the quotient by the kernel of this seminorm gives the separated completion $\widehat{G}$ of $G$. The map $G \rightarrow \widehat{G}$ given by $x \mapsto x, x, \ldots$ is an isometry and hence injective if $\alpha$ itself is a norm; in that case, we call $\widehat{G}$ simply the completion of $G$.

Definition 1.3. Let $A$ be a ring. Consider the following conditions on a multiplicative seminorm $\alpha$ on the additive group of $A$.

(c) We have $\alpha(1) \leq 1$, and for all $g, h \in A$, we have $\alpha(g h) \leq \alpha(g) \alpha(h)$.

(c') We have (c), and for all $g \in A$ we have $\alpha\left(g^{2}\right)=\alpha(g)^{2}$.

$\left(\mathrm{c}^{\prime \prime}\right)$ We have (c), and for all $g, h \in A$, we have $\alpha(g h)=\alpha(g) \alpha(h)$.

We say that $\alpha$ is submultiplicative if it satisfies (c), power-multiplicative if it satisfies $\left(\mathrm{c}^{\prime}\right)$, and multiplicative if it satisfies $\left(\mathrm{c}^{\prime \prime}\right)$. We make some quick observations about these definitions.

(i) For $\alpha$ a submultiplicative seminorm, $\alpha(1)=1$ unless $\alpha$ is identically zero.

(ii) Any power-multiplicative seminorm $\alpha$ satisfies $\alpha\left(g^{n}\right)=\alpha(g)^{n}$ for all $g \in A$ and all nonnegative integers $n$.

(iii) Any multiplicative seminorm is power-multiplicative.

(iv) If $\alpha$ is a submultiplicative seminorm and $\alpha^{\prime}$ is a power-multiplicative seminorm, then $\alpha \geq \alpha^{\prime}$ if and only if $\alpha(a) \geq \alpha^{\prime}(a)$ for all $a \in A$.

ExAmPLE 1.4. For any abelian group $G$, the trivial norm on $G$ sends 0 to 0 and any nonzero $g \in G$ to 1 . For any nonzero ring $A$, the trivial norm on $A$ is submultiplicative in all cases, power-multiplicative if and only if $A$ is reduced, and multiplicative if and only if $A$ is an integral domain. (The trivial norm on the zero ring is multiplicative by virtue of the fact that we do not force $\alpha(1)=1$.)

Definition 1.5. For $A$ a ring equipped with a submultiplicative seminorm $|\cdot|$, we write

$$
\begin{aligned}
\mathfrak{o}_{A} & =\{x \in A:|x| \leq 1\}, \\
\mathfrak{m}_{A} & =\{x \in A:|x|<1\}, \\
\kappa_{A} & =\mathfrak{o}_{A} / \mathfrak{m}_{A} .
\end{aligned}
$$


If $A$ is a field and $|\cdot|$ is a multiplicative norm, then $\kappa_{A}$ is also a field. (The field $\kappa_{A}$ is normally called the residue field of $A$, but we will use this term mostly for something else; see Definition 1.9.)

Definition 1.6. Let $A$ be a ring equipped with a submultiplicative (semi)norm $|\cdot|$. For $r \geq 0$, the $r$-Gauss (semi)norm $|\cdot|_{r}$ on $A[T]$ (for the generator $T$, when this needs to be specified) is the submultiplicative (semi)norm defined by the formula

$$
\left|\sum_{i} x_{i} T^{i}\right|_{r}=\max _{i}\left\{\left|x_{i}\right| r^{i}\right\}
$$

this is multiplicative if $|\cdot|$ is multiplicative (see Lemma 1.7). We refer to the 1-Gauss (semi)norm also simply as the Gauss (semi)norm (or Gauss extension).

Lemma 1.7. Let $A$ be a ring equipped with a multiplicative seminorm $|\cdot|$. Then for any $r \geq 0$, the $r$-Gauss seminorm on $A[T]$ is multiplicative.

Proof. This is evident in case $r=0$, so assume that $r>0$. Let $a=$ $\sum_{j} a_{j} T^{j}, b=\sum_{k} b_{k} T^{k}$ be any two elements of $A[T]$. Choose the smallest indices $j, k$ for which $\left|a_{j}\right| r^{j},\left|b_{k}\right| r^{k}$ are maximized, and put $i=j+k$. The coefficient of $T^{i}$ in $a b$ is then equal to $a_{j} b_{k}$ plus the sum of $a_{j^{\prime}} b_{k^{\prime}}$ over all pairs $\left(j^{\prime}, k^{\prime}\right) \neq(j, k)$ for which $j^{\prime}+k^{\prime}=j+k$. For each such pair, either $j^{\prime}<j$, in which case

$$
\left|a_{j^{\prime}}\right| r^{j^{\prime}}<\left|a_{j}\right| r^{j}, \quad\left|b_{k^{\prime}}\right| r^{k^{\prime}} \leq\left|b_{k}\right| r^{k}
$$

or $k^{\prime}<k$, in which case

$$
\left|a_{j^{\prime}}\right| r^{j^{\prime}} \leq\left|a_{j}\right| r^{j}, \quad\left|b_{k^{\prime}}\right| r^{k^{\prime}}<\left|b_{k}\right| r^{k}
$$

In both cases, we conclude that $\left|a_{j^{\prime}} b_{k^{\prime}}\right|<\left|a_{j} b_{k}\right|$, so the coefficient of $T^{i}$ in $a b$ has norm $\left|a_{j} b_{k}\right|$. This forces $|a b|_{r}=|a|_{r}|b|_{r}$, as desired.

REMARK 1.8. For each $z \in A$, one has a $r$-Gauss seminorm on $A[T]$ for the generator $T-z$. This seminorm can also be constructed by equipping $A[T]$ with the $s$-Gauss norm for some $s \geq \max \{r,|z|\}$, forming the $r$-Gauss extension to $A[T][U]$, and then passing to the quotient norm on $A[T][U] /(U-T+z) \cong A[T]$. 
Definition 1.9. Let $A$ be a ring equipped with a submultiplicative norm $|\cdot|$. The Gel'fand spectrum $\mathcal{M}(A)$ of $A$ is the set of multiplicative seminorms $\alpha$ on $A$ dominated by $|\cdot|$, topologized as a closed subspace of the product $\prod_{a \in A}[0,|a|]$ (see Definition 1.3(iv)). In particular, $\mathcal{M}(A)$ is compact by Tikhonov's theorem. A subbasis of the topology on $\mathcal{M}(A)$ is given by the sets $\{\alpha \in \mathcal{M}(A): \alpha(f) \in I\}$ for each $f \in A$ and each open interval $I \subseteq \mathbb{R}$. For any bounded homomorphism $\phi: A \rightarrow B$ between rings equipped with submultiplicative norms, restriction along $\phi$ defines a continuous map $\phi^{*}: \mathcal{M}(B) \rightarrow \mathcal{M}(A)$; this map is a homeomorphism when $B=\widehat{A}$.

For $\alpha \in \mathcal{M}(A)$, the seminorm $\alpha$ induces a multiplicative norm on the integral domain $A / \alpha^{-1}(0)$ and hence also on $\operatorname{Frac}\left(A / \alpha^{-1}(0)\right)$. The completion of this latter field is the residue field of $\alpha$, denoted $\mathcal{H}(\alpha)$. (Note that $\mathcal{H}(\alpha)$ itself has a "residue field" $\kappa_{\mathcal{H}(\alpha)}$ in the sense of Definition 1.5.)

THEOREM 1.10. Let $A$ be a nonzero ring equipped with a submultiplicative norm $|\cdot|$. Then $\mathcal{M}(A) \neq \emptyset$.

Proof. Replace $A$ by its completion, and then apply [2, Theorem 1.2.1].

Definition 1.11. Let $A$ be a ring equipped with a submultiplicative norm $|\cdot|$. Let $|\cdot|_{\text {sup }}: \prod_{\alpha \in \mathcal{M}(A)} \mathcal{H}(\alpha) \rightarrow[0,+\infty]$ denote the supremum of the norms on the $\mathcal{H}(\alpha)$. Let $P$ be the inverse image of $[0,+\infty)$ under $|\cdot|_{\text {sup }}$; then $|\cdot|_{\text {sup }}$ defines a power-multiplicative norm on $P$. The diagonal map $A \rightarrow \prod_{\alpha \in \mathcal{M}(A)} \mathcal{H}(\alpha)$ then factors through a bounded homomorphism $A \rightarrow$ $P$, called the Gel'fand transform of $A$.

Lemma 1.12. Let $A$ be a ring equipped with a submultiplicative norm $|\cdot|$. Then the restriction of $|\cdot|_{\text {sup }}$ to $A$ along the Gel'fand transform computes the spectral seminorm $|a|_{\mathrm{sp}}=\lim _{s \rightarrow \infty}\left|a^{s}\right|^{1 / s}$ on $A$.

Proof. See [2, Theorem 1.3.1].

REMARK 1.13. Let $A$ be a ring equipped with a submultiplicative norm $|\cdot|$. Let $I \subset A$ be the kernel of the spectral seminorm. Choose a closed subset $X$ of $\mathcal{M}(A)$. Let $S$ be the multiplicative subset of $a \in A / I$ for which $\inf \{\alpha(a): \alpha \in X\}>0$. Put $B=S^{-1}(A / I)$, equipped with the supremum norm over $X$; then the map $A \rightarrow B$ is a bounded homomorphism inducing a homeomorphism of $\mathcal{M}(B)$ with a closed subset of $\mathcal{M}(A)$ containing $X$. In many (but not all) cases, this closed subset equals $X$; for instance, this occurs for the sets described in Definition 1.14 below. This is related to the 
nonarchimedean analogues of the notions of holomorphically convex and meromorphically convex sets (see [2, Section 2.6]).

The following examples of the previous construction occur when comparing nonarchimedean analytic geometry with formal geometry or rigid analytic geometry (as explained in [3, Section 1.6]).

Definition 1.14. Let $A$ be a ring equipped with a submultiplicative norm $|\cdot|$. A Weierstrass subspace of $\mathcal{M}(A)$ is a closed subspace of the form

$$
U=\left\{\alpha \in \mathcal{M}(A): \alpha\left(f_{i}\right) \leq p_{i}(i=1, \ldots, n)\right\}
$$

for some $f_{1}, \ldots, f_{n} \in A$ and some $p_{1}, \ldots, p_{n}>0$. A Laurent subspace of $\mathcal{M}(A)$ is a closed subspace of the form

$$
U=\left\{\alpha \in \mathcal{M}(A): \alpha\left(f_{i}\right) \leq p_{i}, \alpha\left(g_{j}\right) \geq q_{j}(i=1, \ldots, m ; j=1, \ldots, n)\right\}
$$

for some $f_{1}, \ldots, f_{m}, g_{1}, \ldots, g_{n} \in A$ and some $p_{1}, \ldots, p_{m}, q_{1}, \ldots, q_{n}>0$; the Laurent subspaces form a basis of closed neighborhoods for the topology of $\mathcal{M}(A)$. A rational subspace of $\mathcal{M}(A)$ is a closed subspace of the form

$$
U=\left\{\alpha \in \mathcal{M}(A): \alpha\left(f_{i}\right) \leq p_{i} \alpha(g)(i=1, \ldots, n)\right\}
$$

for some $f_{1}, \ldots, f_{n}, g \in A$ which generate the unit ideal in $\widehat{A}$ and some $p_{1}, \ldots, p_{n}>0$; we may assume without loss of generality that $f_{n}=g, p_{n}=1$. The intersection of rational subspaces is rational [7, Proposition 7.2.3/7]; consequently, any Laurent subspace is rational.

We will say that a continuous map between Gel'fand spectra is strongly continuous if the inverse image of any Weierstrass (resp., Laurent, rational) subspace is a finite union of Weierstrass (resp., Laurent, rational) subspaces. For instance, the restriction map along a bounded homomorphism is strongly continuous.

REMARK 1.15. It is easy to see that a Weierstrass or Laurent subspace of $\mathcal{M}(\widehat{A})$ remains Weierstrass or Laurent when viewed as a subset of $\mathcal{M}(A)$. This is also true for rational subspaces, but the argument is a bit less immediate. Let

$$
U=\left\{\alpha \in \mathcal{M}(\widehat{A}): \alpha\left(f_{i}\right) \leq p_{i} \alpha(g)(i=1, \ldots, n)\right\}
$$


be a rational subspace of $\mathcal{M}(\widehat{A})$ for some $f_{1}, \ldots, f_{n}, g \in \widehat{A}$ which generate the unit ideal and some $p_{1}, \ldots, p_{n}>0$. Choose $u_{1}, \ldots, u_{n}, v \in \widehat{A}$ for which $u_{1} f_{1}+\cdots+u_{n} f_{n}+v g=1$. For $\alpha \in U$, we then have

$$
1 \leq \max \left\{\alpha\left(u_{1} f_{1}\right), \ldots, \alpha\left(u_{n} f_{n}\right), \alpha(v g)\right\} \leq \max \left\{\left|u_{1}\right| p_{1}, \ldots,\left|u_{n}\right| p_{n},|v|\right\} \alpha(g) .
$$

Choose $\epsilon \in(0,1)$ so that $\epsilon \max \left\{\left|u_{1}\right| p_{1}, \ldots,\left|u_{n}\right| p_{n},|v|\right\}<1$; then $\alpha(g) \geq \epsilon$ for all $\alpha \in U$. Choose $f_{1}^{\prime}, \ldots, f_{n}^{\prime}, g^{\prime} \in A$ with

$$
\left|f_{1}-f_{1}^{\prime}\right|<p_{1} \epsilon, \ldots,\left|f_{n}-f_{n}^{\prime}\right|<p_{n} \epsilon,\left|g-g^{\prime}\right|<\epsilon .
$$

On one hand,

$$
\left|u_{1} f_{1}^{\prime}+\cdots+u_{n} f_{n}^{\prime}+v g^{\prime}-1\right| \leq \epsilon \max \left\{\left|u_{1}\right| p_{1}, \ldots,\left|u_{n}\right| p_{n},|v|\right\}<1,
$$

so $f_{1}^{\prime}, \ldots, f_{n}^{\prime}, g^{\prime}$ still generate the unit ideal in $\widehat{A}$. On the other hand,

$$
U=\left\{\alpha \in \mathcal{M}(\widehat{A}): \alpha\left(f_{i}^{\prime}\right) \leq p_{i} \alpha\left(g^{\prime}\right)(i=1, \ldots, n)\right\},
$$

so $U$ is a rational subspace of $\mathcal{M}(A)$. (Note that we cannot hope to ensure that $f_{1}^{\prime}, \ldots, f_{n}^{\prime}, g^{\prime}$ generate the unit ideal in $A$ itself.)

Definition 1.16. Let $A, B, C$ be rings equipped with submultiplicative norms $|\cdot|_{A},|\cdot|_{B},|\cdot|_{C}$. Let $A \rightarrow B$ and $A \rightarrow C$ be bounded homomorphisms. Define the product seminorm $|\cdot|_{B \otimes C}$ on $B \otimes_{A} C$ by taking $|f|_{B \otimes C}$ to be the infimum of $\max _{i}\left\{\left|b_{i}\right|_{B}\left|c_{i}\right|_{C}\right\}$ over all presentations $\sum_{i} b_{i} \otimes c_{i}$ of $f$. Let $B \widehat{\otimes}_{A} C$ be the separated completion of $B \bigotimes_{A} C$ for the product seminorm.

It is sometimes difficult to tell whether $B \widehat{\otimes}_{A} C$ is nonzero; we get around this using the following definition. By a splitting of $\iota: A \rightarrow B$, we will mean a bounded homomorphism $\pi: B \rightarrow A$ of $A$-modules with $\pi \circ \iota=\mathrm{id}_{A}$. We say that $\iota$ is split if it admits a splitting; this implies that $|\cdot|_{A}$ is equivalent to the restriction of $|\cdot|_{B}$.

LEMMA 1.17. Let $A, B, C$ be rings equipped with submultiplicative norms $|\cdot|_{A},|\cdot|_{B},|\cdot|_{C}$. Let $A \rightarrow B$ and $A \rightarrow C$ be bounded homomorphisms. Then $|\cdot|_{B \otimes C}$ induces a submultiplicative norm on $B \widehat{\bigotimes}_{A} C$. In addition, if $\iota: A \rightarrow B$ is split, then so is $C \rightarrow B \widehat{\bigotimes}_{A} C$.

Proof. From the presentation $1=1 \otimes 1$, we read off that $|1|_{B \otimes C} \leq 1$. For $f=\sum_{i} b_{i} \otimes c_{i}, f^{\prime}=\sum_{j} b_{j}^{\prime} \otimes c_{j}^{\prime} \in B \otimes_{A} C$, we may write $f f^{\prime}=\sum_{i, j}\left(b_{i} b_{j}^{\prime}\right) \otimes$ $\left(c_{i} c_{j}^{\prime}\right)$ and deduce that

$$
\left|f f^{\prime}\right|_{B \otimes C} \leq \max _{i, j}\left\{\left|b_{i} b_{j}^{\prime}\right|_{B}\left|c_{i} c_{j}^{\prime}\right|_{C}\right\} \leq \max _{i}\left\{\left|b_{i}\right|_{B}\left|c_{i}\right|_{C}\right\} \max _{j}\left\{\left|b_{j}^{\prime}\right|_{B}\left|c_{j}^{\prime}\right|_{C}\right\} .
$$


Taking the infimum over all presentations of $f$ and $f^{\prime}$ yields $\left|f f^{\prime}\right|_{B \otimes C} \leq$ $|f|_{B \otimes C}\left|f^{\prime}\right|_{B \otimes C}$, so $|\cdot|_{B \otimes C}$ is a submultiplicative norm on $B \widehat{\otimes}_{A} C$.

Suppose further that $\pi: B \rightarrow A$ is a splitting of $\iota$. By tensoring $\pi$ over $A$ with $C$, we obtain a bounded projection $B \otimes_{A} C \rightarrow C$ of $C$-modules with $C \rightarrow B \otimes_{A} C \rightarrow C$ being the identity. By continuity, we obtain a projection $B \widehat{\bigotimes}_{A} C \rightarrow C$ with the same effect, so $C \rightarrow B \widehat{\bigotimes}_{A} C$ is split.

REMARK 1.18. Keep in mind that $|\cdot|_{B \otimes C}$ need not be multiplicative even if $|\cdot|_{A},|\cdot|_{B},|\cdot|_{C}$ are multiplicative. For example, if $K$ is a quadratic extension of the $p$-adic field $\mathbb{Q}_{p}$, then $K \bigotimes_{\mathbb{Q}_{p}} K \cong K \widehat{\bigotimes}_{\mathbb{Q}_{p}} K$ splits as a direct sum of two copies of $K$.

Lemma 1.19. Let $A, B$ be rings equipped with submultiplicative norms $|\cdot|_{A},|\cdot|_{B}$. Let $\phi: A \rightarrow B$ be a split homomorphism. Then the map $\phi^{*}$ is surjective.

Proof. For any $\alpha \in \mathcal{M}(A)$, the homomorphism $\mathcal{H}(\alpha) \rightarrow B \widehat{\bigotimes}_{A} \mathcal{H}(\alpha)$ is split by Lemma 1.17; in particular, the target is nonzero and carries a submultiplicative norm. By Theorem 1.10, there exists some $\beta \in$ $\mathcal{M}\left(B \widehat{\bigotimes}_{A} \mathcal{H}(\alpha)\right)$. The seminorms $\alpha$ and $\beta \circ \phi$ on $A$ then coincide.

Lemma 1.20. Let $A, B, C$ be rings equipped with submultiplicative norms. Then the map $\mathcal{M}\left(B \widehat{\bigotimes}_{A} C\right) \rightarrow \mathcal{M}(B) \times_{\mathcal{M}(A)} \mathcal{M}(C)$ is surjective.

Proof. Choose $\beta \in \mathcal{M}(B), \gamma \in \mathcal{M}(C)$ having the same image $\alpha$ in $\mathcal{M}(A)$. Using the existence of Schauder bases for Banach modules over nonarchimedean fields, it can be shown that the completed tensor product of any two nonzero Banach modules over $\mathcal{H}(\alpha)$ is nonzero (see [19, Lemma 1.3.11]). In particular, $D=\mathcal{H}(\beta) \widehat{\bigotimes}_{\mathcal{H}(\alpha)} \mathcal{H}(\gamma)$ is nonzero, so by Theorem 1.10, there exists some $\delta \in \mathcal{M}(D)$. The restrictions of $\delta$ to $\mathcal{H}(\beta), \mathcal{H}(\gamma)$ give back $\beta, \gamma$, so the same is true of the restrictions to $B, C$.

\section{$\S 2$. Nonarchimedean geometry of polynomial rings}

To illustrate the results we have concerning the nonarchimedean geometry of Witt vectors, we first describe the analogous statements relating the nonarchimedean analytic spaces associated to a ring $R$ and the polynomial ring $R[T]$.

Hypothesis 2.1. Throughout Section 2, let $R$ be a ring equipped with a submultiplicative norm $|\cdot|$, and equip $R[T]$ with the Gauss norm. 
TheOREM 2.2. For $\alpha \in \mathcal{M}(R)$, let $\lambda(\alpha) \in \mathcal{M}(R[T])$ be the Gauss extension of $\alpha$. For $\beta \in \mathcal{M}(R[T])$, let $\mu(\beta) \in \mathcal{M}(R)$ be the restriction of $\beta$ along $R \rightarrow R[T]$.

(a) The maps $\lambda$ and $\mu$ are strongly continuous and monotonic.

(b) For all $\alpha \in \mathcal{M}(R),(\mu \circ \lambda)(\alpha)=\alpha$.

(c) For all $\beta \in \mathcal{M}(R[T]),(\lambda \circ \mu)(\beta) \geq \beta$.

Proof. The map $\mu$ is defined as a restriction and hence is strongly continuous. For $f=\sum_{i=0}^{m} f_{i} T^{i} \in R[T]$ and $\epsilon>0$, we have

$$
\begin{aligned}
& \{\alpha \in \mathcal{M}(R): \lambda(\alpha)(f)>\epsilon\}=\bigcup_{i=0}^{m-1}\left\{\alpha \in \mathcal{M}(R): \alpha\left(f_{i}\right)>\epsilon\right\}, \\
& \{\alpha \in \mathcal{M}(R): \lambda(\alpha)(f)<\epsilon\}=\bigcap_{i=0}^{m-1}\left\{\alpha \in \mathcal{M}(R): \alpha\left(f_{i}\right)<\epsilon\right\},
\end{aligned}
$$

so $\lambda$ is continuous. Similarly, the inverse image of a Weierstrass (resp., Laurent) subspace of $\mathcal{M}(R[T])$ is a finite union of Weierstrass (resp., Laurent) subspaces of $\mathcal{M}(R)$. Now let

$$
U=\left\{\beta \in \mathcal{M}(R[T]): \beta\left(f_{i}\right) \leq p_{i} \beta(g)(i=1, \ldots, n)\right\}
$$

be a rational subspace of $\mathcal{M}(R[T])$ for some $f_{1}, \ldots, f_{n}, g \in R[T]$ generating the unit ideal in the completion of $R[T]$ and some $p_{1}, \ldots, p_{n}>0$. Write $f_{i}=$ $\sum_{j=0}^{m} f_{i j} T^{j}$ and $g=\sum_{j=0}^{m} g_{j} T^{j}$; then the $f_{i j}$ and $g_{j}$ together must generate the unit ideal (in fact, only the $f_{i 0}$ and $g_{0}$ are needed). We may write

$$
\begin{aligned}
\lambda^{-1}(U)= & \left\{\alpha \in \mathcal{M}(R): \max _{i, j}\left\{\alpha\left(f_{i j}\right) / p_{i}\right\} \leq \max _{j}\left\{\alpha\left(g_{j}\right)\right\}\right\} \\
= & \bigcup_{l=0}^{m}\left\{\alpha \in \mathcal{M}(R): \alpha\left(f_{i j}\right) \leq p_{i} \alpha\left(g_{l}\right), \alpha\left(g_{j}\right) \leq \alpha\left(g_{l}\right)\right. \\
& (i=1, \ldots, n ; j=0, \ldots, m)\},
\end{aligned}
$$

which is a finite union of rational subspaces of $\mathcal{M}(R)$. Since monotonicity is evident, this yields (a).

Of the remaining assertions, (b) is trivial, while (c) holds because $(\lambda \circ$ $\mu)(\beta)\left(f_{i} T^{i}\right) \geq \beta\left(f_{i} T^{i}\right)$ for any $f_{i} \in R$ and any nonnegative integer $i$.

The following construction is described by Berkovich [2, Remark 6.1.3(ii)]. 
Lemma 2.3. For any $\beta \in \mathcal{M}(R[T])$ and any $t \in[0,1]$, the function $H(\beta, t)$ : $R[T] \rightarrow[0,+\infty)$ defined by

$$
H(\beta, t)(f)=\max _{i}\left\{t^{i} \beta\left(\frac{1}{i !} \frac{d^{i}}{d T^{i}}(f)\right)\right\}
$$

is a multiplicative seminorm on $R[T]$ dominated by the Gauss norm.

Proof. It is evident that (2.3.1) defines a seminorm dominated by the Gauss norm. Submultiplicativity follows from the Leibniz rule in the form

$$
\frac{1}{i !} \frac{d^{i}}{d T^{i}}(g h)=\sum_{j+k=i} \frac{1}{j !} \frac{d^{j}}{d T^{j}}(g) \frac{1}{k !} \frac{d^{k}}{d T^{k}}(h) .
$$

To check multiplicativity, we must check that for $g, h \in R[T]$, we have $H(\beta, t)(g h) \geq H(\beta, t)(g) H(\beta, t)(h)$. Choose the minimal indices $j, k$ achieving the maxima in (2.3.1) for $f=g, h$. Then in (2.3.2) for $i=j+k$, the maximum $\beta$-norm among the summands on the right-hand side of (2.3.2) is achieved only by the pair $(j, k)$ (as in the proof of Lemma 1.7). Since $\beta$ is multiplicative, we obtain

$$
t^{i} \beta\left(\frac{1}{i !} \frac{d^{i}}{d T^{i}}(g h)\right)=t^{j} \beta\left(\frac{1}{j !} \frac{d^{j}}{d T^{j}}(g)\right) t^{k} \beta\left(\frac{1}{k !} \frac{d^{k}}{d T^{k}}(h)\right),
$$

proving the desired result.

When $\beta$ is a Gauss seminorm, we can describe $H(\beta, t)$ explicitly.

LEMma 2.4. Let $\beta \in \mathcal{M}(R[T])$ be the $r$-Gauss seminorm for the generator $T-x$ for some $x \in R$ and some $r \in[0,1]$. Then for $t \in[0,1], H(\beta, t)$ is the $\max \{t, r\}$-Gauss seminorm for the generator $T-x$. In particular, $H(\beta, 1)$ is the Gauss norm.

Proof. We first check the claim for $t \geq r$. Let $\gamma$ be the $t$-Gauss seminorm for the generator $T-x$. Write $f \in R[T]$ as $\sum_{j} f_{j}(T-x)^{j}$ with $f_{j} \in R$, so that $\gamma(f)=\max _{j}\left\{t^{j}\left|f_{j}\right|\right\}$. Since $t \geq r$, we have

$$
\begin{aligned}
t^{i} \beta\left(\frac{1}{i !} \frac{d^{i}}{d T^{i}}(f)\right) & =t^{i} \max _{j \geq i}\left\{\beta\left(\left(\begin{array}{l}
j \\
i
\end{array}\right) f_{j}(T-x)^{j-i}\right)\right\} \\
& \leq \max _{j \geq i}\left\{t^{i} r^{j-i}\left|f_{j}\right|\right\} \leq \gamma(f) .
\end{aligned}
$$


It follows that $\gamma(f) \geq H(\beta, t)(f)$. In contrast, for each nonnegative integer $i$,

$$
t^{i} \beta\left(\frac{1}{i !} \frac{d^{i}}{d T^{i}}(f)\right) \geq t^{i}\left|f_{i}\right|
$$

because the constant term of $d^{i} f / d T^{i}$ is $i ! f_{i}$. It follows that $H(\beta, t)(f)=$ $\gamma(f)$.

In case $t<r$, on one hand we have $H(\beta, t) \geq \beta$ by taking $i=0$ on the right-hand side of (2.3.1), and on the other hand we have $H(\beta, t) \leq$ $H(\beta, r)=\beta$ because the right-hand side of (2.3.1) is monotone in $t$. Hence, $H(\beta, t)=\beta$.

Theorem 2.5. The map $H: \mathcal{M}(R[T]) \times[0,1] \rightarrow \mathcal{M}(R[T])$ is continuous and has the following additional properties.

(a) For $\beta \in \mathcal{M}(R[T]), H(\beta, 0)=\beta$.

(b) For $\beta \in \mathcal{M}(R[T]), H(\beta, 1)=(\lambda \circ \mu)(\beta)$.

(c) For $\beta \in \mathcal{M}(R[T])$ and $t \in[0,1], \mu(H(\beta, t))=\mu(\beta)$.

(d) For $\beta \in \mathcal{M}(R[T])$ and $s, t \in[0,1], H(H(\beta, s), t)=H(\beta, \max \{s, t\})$.

Proof. The continuity of $H$ is evident from (2.3.1), since the maximum on the right-hand side runs over only finitely many terms. Of the other properties, (a) and (c) are evident from (2.3.1). To check (b), let $\gamma \in \mathcal{M}(R[T])$ be the Gauss norm. For $\beta \in \mathcal{M}(R[T]), \beta \leq \gamma$ and so $H(\beta, 1) \leq H(\gamma, 1)=\gamma$ by Lemma 2.4; on the other hand, taking $t=1$ in (2.4.1) yields $H(\beta, 1) \geq \gamma$. (We can also deduce (b) from Lemma 2.4 using Remark 2.8 below.)

To check $(\mathrm{d})$, observe that

$$
\begin{aligned}
H(H(\beta, s), t) & =\max _{j}\left\{t^{j} \max _{k}\left\{s^{k} \beta\left(\frac{1}{k !} \frac{d^{k}}{d T^{k}}\left(\frac{1}{j !} \frac{d^{j}}{d T^{j}}(f)\right)\right)\right\}\right\} \\
& =\max _{j, k}\left\{t^{j} s^{k} \beta\left(\left(\begin{array}{c}
j+k \\
j
\end{array}\right) \frac{1}{(j+k) !} \frac{d^{j+k}}{d T^{j+k}}(f)\right)\right\} \\
& =\max _{i}\left\{\beta\left(\frac{1}{i !} \frac{d^{i}}{d T^{i}}(f)\right) \max _{j+k=i}\left\{t^{j} s^{k} \beta\left(\left(\begin{array}{c}
j+k \\
j
\end{array}\right)\right)\right\}\right\} .
\end{aligned}
$$

Since $\beta$ is a norm, $t^{j} s^{k} \beta\left(\left(\begin{array}{c}j+k \\ j\end{array}\right)\right) \leq \max \{s, t\}^{i}$, with equality if $s \geq t$ and $(j, k)=(0, i)$, or if $s \leq t$ and $(j, k)=(i, 0)$. This proves $(\mathrm{d})$.

Corollary 2.6. Each subset of $\mathcal{M}(R)$ has the same homotopy type as its inverse image under $\mu$. 
REMARK 2.7. From Theorem 2.5, (b) and (d), it follows that for $\alpha \in$ $\mathcal{M}(R)$ and $t \in[0,1], H(\lambda(\alpha), t)=\lambda(\alpha)$. This can also be seen more directly: note that $H(\lambda(\alpha), t) \geq \lambda(\alpha)$ from (2.3.1), while the reverse inequality follows from Theorem 2.5(c) plus Theorem 2.2(c).

REMARK 2.8. One can give an alternate proof of Lemma 2.3 using Lemma 2.4, as follows. Let $\tilde{\beta} \in \mathcal{M}(R[U][T])$ be the restriction of $\beta$ along

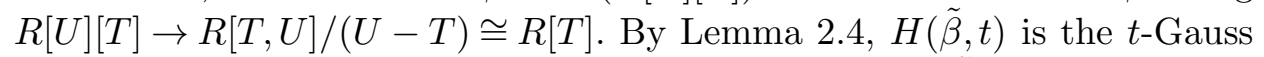
seminorm for the generator $T-U$. The restriction of $H(\tilde{\beta}, t)$ along $R[T] \rightarrow$ $R[U][T]$ is $H(\beta, t)$, so the latter is a multiplicative seminorm.

One can go further and take this construction as the definition of $H(\beta, t)$, modifying the proof of Theorem 2.5 accordingly. We will not write out the details explicitly, but they will be shadowed in the context of Witt vectors where no good analogue of (2.3.1) is available (see, e.g., the proof of Theorem 7.8).

REMARK 2.9. One may view $\mathcal{M}(R[T])$ as a closed cylinder of radius 1 over $\mathcal{M}(R)$ and view $\lambda$ as the section taking each point of $\mathcal{M}(R)$ to the generic point of its fiber. In this language, Theorem 2.5 states that $\mathcal{M}(R[T])$ can be uniformly contracted onto the image of $\lambda$; in particular, each fiber of $\mu$ is contractible. We further elucidate the structure of the fibers of $\mu$ by studying the domination relation (see Theorem 2.11 and Remark 2.29).

Definition 2.10. For $\beta \in \mathcal{M}(R[T])$, the set of $s \in[0,1]$ for which $H(\beta, s)=\beta$ is nonempty (because it contains 0 ) and closed (by continuity), so it has a greatest element. This element is called the radius of $\beta$ and is denoted $r(\beta)$; this terminology is justified by the fact that the $r$-Gauss norm has radius $r$ (see also Remark 2.16).

Theorem 2.11. For $\beta, \gamma \in \mathcal{M}(R[T])$ satisfying $\mu(\beta)=\mu(\gamma)$ and $\beta \geq \gamma$, $\beta=H(\gamma, r(\beta))$.

Proof. Put $\alpha=\mu(\beta)=\mu(\gamma)$ and put $K=\mathcal{H}(\alpha)$; then identify $\beta$, $\gamma$ with the corresponding points in $\mathcal{M}(K[T])$. These identifications are compatible with the formation of $H(\cdot, t)$; in particular, they do not change the radius of $\beta$. It thus suffices to check the case $R=K$, for which we rely on some analysis of $\mathcal{M}(K[T])$ (see Lemma 2.22 below).

Corollary 2.12. For $\beta, \gamma \in \mathcal{M}(R[T])$ satisfying $\mu(\beta)=\mu(\gamma)$ and $\beta \geq \gamma$, we have $r(\beta) \geq r(\gamma)$, with equality if and only if $\beta=\gamma$. 
Proof. For $t \in[0, r(\gamma)]$, by Theorem 2.11 and Theorem 2.5(d), we have

$$
H(\beta, t)=H(H(\gamma, r(\beta)), t)=H(H(\gamma, t), r(\beta))=H(\gamma, r(\beta))=\beta,
$$

so $r(\beta) \geq r(\gamma)$. If equality holds, then $\gamma=H(\gamma, r(\gamma))=H(\gamma, r(\beta))=\beta$.

In order to complete the proof of Theorem 2.11, we must study $\mathcal{M}(K[T])$ when $K$ is a complete nonarchimedean field. In case $K$ is algebraically closed, this was done by Berkovich [2, Section 1.4] (see also [1, Proposition 1.1]). The general case can be found in [22, Section 2.2], where it is treated by reduction to the algebraically closed case. We give here some direct arguments in terms of the map $H$.

Hypothesis 2.13. For the remainder of Section 2, let $K$ be a field complete for a multiplicative norm $\alpha$, let $\mathfrak{o}$ be the valuation subring of a completed algebraic closure $\mathbb{C}$ of $K$, and equip both $K[T]$ and $\mathbb{C}[T]$ with the Gauss norms.

REMARK 2.14. It is not hard to check that $\mathcal{M}(K[T])$ is the quotient of $\mathcal{M}(\mathbb{C}[T])$ by the action of the group $\operatorname{Aut}(\mathbb{C} / K)$ of continuous automorphisms of $\mathbb{C}$ over $K$ (see [2, Proposition 1.3.5]). We will not use this fact explicitly, but it is useful to keep in mind.

Definition 2.15. For $z \in \mathfrak{o}$ and $r \in[0,1]$, let $\tilde{\beta}_{z, r}$ be the $r$-Gauss norm on $\mathbb{C}[T]$ for the generator $T-z$, and let $\beta_{z, r}$ denote the restriction of $\tilde{\beta}_{z, r}$ to $K[T]$. If $z^{\prime} \in \mathfrak{o}$ satisfies $\alpha\left(z^{\prime}-z\right) \leq r$, then $\tilde{\beta}_{z^{\prime}, r}=\tilde{\beta}_{z, r}$; consequently, if $r>0$, we always have $\beta_{z, r}=\beta_{z^{\prime}, r}$ for some $z^{\prime} \in \mathfrak{o}$ which is integral over $K$ (since such $z^{\prime}$ are dense in $\mathfrak{o}$ ).

REMARK 2.16. If the norm on $K$ is nontrivial, then the seminorm $\tilde{\beta}_{z, r}$ can be identified with the supremum norm over the closed disk in $\mathbb{C}$ of center $z$ and radius $r$. Although this fact can be proved directly, it will be convenient for us not to deduce it until after making our principal arguments (see Corollary 2.25).

Lemma 2.17. For $z \in \mathfrak{o}$ and $r, s \in[0,1], \beta_{z, r} \geq \beta_{z, s}$ if and only if $r \geq s$.

Proof. If $r \geq s$, then evidently $\beta_{z, r} \geq \beta_{z, s}$. It remains to show that if $r>s$, then $\beta_{z, r} \neq \beta_{z, s}$. It suffices to do this when $s>0$, as when $s=0$ we can argue that $\beta_{z, r}>\beta_{z, r^{\prime}} \geq \beta_{z, 0}$ for any $r^{\prime} \in(0, r)$.

Suppose, then, that $s>0$. Choose $z^{\prime} \in \mathfrak{o}$ integral over $K$ with $\alpha(z-$ $\left.z^{\prime}\right) \leq s$, so that $\beta_{z, r}=\beta_{z^{\prime}, r}, \beta_{z, s}=\beta_{z^{\prime}, s}$. Let $P(T)=\prod_{i=1}^{m}\left(T-z_{i}\right)$ be the 
minimal polynomial of $z^{\prime}$ over $K$; then $\tilde{\beta}_{z^{\prime}, r}\left(T-z_{i}\right) \geq \tilde{\beta}_{z^{\prime}, s}\left(T-z_{i}\right)$ for each $i$, with strict inequality when $z_{i}=z^{\prime}$. Hence, $\beta_{z, r}(P)=\tilde{\beta}_{z^{\prime}, r}(P)>\tilde{\beta}_{z^{\prime}, s}(P)=$ $\beta_{z, s}(P)$, so $\beta_{z, r} \neq \beta_{z, s}$, as desired.

Corollary 2.18. For $z \in \mathfrak{o}$ and $r \in[0,1], r\left(\beta_{z, r}\right)=r$.

Proof. By Lemma 2.4, we have $H\left(\beta_{z, r}, s\right)=\beta_{z, \max \{r, s\}}$ for $s \in[0,1]$. The claim then follows from Lemma 2.17.

Lemma 2.19. For $z, z^{\prime} \in \mathfrak{o}$ and $r \in[0,1]$, the following are equivalent.

(a) We have $\beta_{z, r}=\beta_{z^{\prime}, r}$.

(b) We have $\beta_{z, r} \geq \beta_{z^{\prime}, r}$.

(c) We have $\beta_{z, r} \geq \beta_{z^{\prime}, 0}$.

(d) There exists $\tau \in \operatorname{Aut}(\mathbb{C} / K)$ for which $\alpha\left(\tau(z)-z^{\prime}\right) \leq r$.

Proof. It is clear that $(\mathrm{d}) \Longrightarrow(\mathrm{a}) \Longrightarrow(\mathrm{b}) \Longrightarrow(\mathrm{c})$, so it suffices to check that $(\mathrm{c}) \Longrightarrow(\mathrm{d})$. For this, we may reduce to the case $r>0$ (using the completeness of $\mathbb{C}$ and the compactness of Aut $(\mathbb{C} / K)$ ). Assume (c); then choose $y \in \mathfrak{o}$ integral over $K$ with $\alpha(y-z) \leq r$, so that $\tilde{\beta}_{y, r}=\tilde{\beta}_{z, r}$. Let $P(T)=\prod_{i=1}^{m}\left(T-y_{i}\right)$ be the minimal polynomial of $y$ over $K$, with the roots ordered so that the sequence $\alpha\left(y_{i}-z^{\prime}\right)$ is nondecreasing.

If (d) fails, then $\alpha\left(y_{i}-z^{\prime}\right)>r$ for $i=1, \ldots, m$. Since $\alpha\left(y_{i}-z^{\prime}\right) \geq \alpha\left(y_{1}-\right.$ $\left.z^{\prime}\right)$, we have $\alpha\left(y_{i}-z^{\prime}\right)=\max \left\{\alpha\left(y_{i}-z^{\prime}\right), \alpha\left(y_{1}-z^{\prime}\right)\right\} \geq \alpha\left(y_{i}-y_{1}\right)$. Hence, $\max \left\{r, \alpha\left(y_{1}-y_{i}\right)\right\} \leq \alpha\left(y_{i}-z^{\prime}\right)$ with strict inequality for $i=1$, so

$$
\begin{aligned}
\beta_{z, r}(P) & =\beta_{y, r}(P)=\tilde{\beta}_{y_{1}, r}(P) \\
& =\prod_{i=1}^{m} \max \left\{r, \alpha\left(y_{1}-y_{i}\right)\right\}<\prod_{i=1}^{m} \alpha\left(z^{\prime}-y_{i}\right)=\beta_{z^{\prime}, 0}(P),
\end{aligned}
$$

a contradiction. Thus, (d) holds, as desired (see also [22, Lemma 2.2.5]).

The key to the proof of Theorem 2.11 is the following calculation in the spirit of Remark 2.8 .

Lemma 2.20. For $\beta \in \mathcal{M}(K[T])$ and $s \in(r(\beta), 1]$, there exists $z \in \mathfrak{o}$ for which $H(\beta, s)=\beta_{z, s}$.

Proof. Let $S$ be the set of $s \in[0,1]$ for which we can find $z \in \mathfrak{o}$ (depending on $s$ ) satisfying $H(\beta, s)=\beta_{z, s}$. The set $S$ is nonempty because $1 \in S$; it is up-closed because $H\left(\beta_{z, r}, s\right)=\beta_{z, \max \{r, s\}}$ by Lemma 2.4 and $H(H(\beta, r), s)=$ $H(\beta, \max \{r, s\})$ by Theorem $2.5(\mathrm{~d})$. Put $r=\inf S$. To prove the lemma, it suffices to check that $r(\beta) \geq r$. 
Let $\mathbb{C}^{\prime}$ be a completed algebraic closure of $\mathcal{H}(\beta)$, fix a continuous embedding of $\mathbb{C}$ into $\mathbb{C}^{\prime}$, and let $x \in \mathbb{C}^{\prime}$ be the image of $T$ under the map $K[T] \rightarrow$ $\mathcal{H}(\beta)$. For $s \in[0,1]$, let $\gamma_{x, s}$ denote the $s$-Gauss norm on $\mathcal{H}(\beta)[T]$ for the generator $T-x$, so that $H(\beta, s)$ is the restriction of $\gamma_{x, s}$ to $K[T]$. By Lemma 2.19 and the stability of $\mathbb{C}$ under $\operatorname{Aut}\left(\mathbb{C}^{\prime} / \mathcal{H}(\beta)\right)$, for $z \in \mathfrak{o}, H(\beta, s)=$ $\beta_{z, s}$ if and only if there exists $\tau \in \operatorname{Aut}(\mathbb{C} / K)$ for which $\alpha(\tau(z)-x) \leq s$. It follows that for $s \in[0, r), \gamma_{x, s}(T-z)=\max \{s, \alpha(z-x)\}$ is independent of $s$. Since every element of $K[T]$ factors in $\mathbb{C}[T]$ as a scalar times a product of linear polynomials, the restriction of $\gamma_{x, s}$ to $K[T]$ is constant over $s \in[0, r)$. Hence, $r(\beta) \geq r$, as desired.

From the proof of Lemma 2.20, we also read off the following observation.

Corollary 2.21. Suppose that $\beta \in \mathcal{M}(K[T])$ is such that $\beta \neq \beta_{z, r}$ for all $z \in \mathfrak{o}$ and all $r \in[0,1]$. Then for each $y \in K[T]$, for any sufficiently small $s \in(r(\beta), 1]$ (depending on $y), H(\beta, s)(y)=\beta(y)$.

With this, we may now complete the proof of Theorem 2.11.

Lemma 2.22. Theorem 2.11 holds for $R=K$.

Proof. If $r(\beta)=1$, then $\beta=H(\beta, 1)=H(\gamma, 1)$ by Theorem 2.5(b). If $r(\gamma)=1$, then by Theorem 2.5 (b) again, $\beta \geq \gamma=H(\gamma, 1)=H(\beta, 1) \geq \beta$, and so $\beta=H(\gamma, 1)$. It is thus safe to assume that $r(\beta), r(\gamma)<1$.

For each $s \in(\max \{r(\beta), r(\gamma)\}, 1]$, by Lemma 2.20 we have $H(\beta, s)=\beta_{z, s}$, $H(\gamma, s)=\beta_{z^{\prime}, s}$ for some $z, z^{\prime} \in \mathfrak{o}$. Since $\beta \geq \gamma$ implies that $H(\beta, s) \geq H(\gamma, s)$, we have $\beta_{z, s} \geq \beta_{z^{\prime}, s}$, but by Lemma 2.19 , this forces $\beta_{z, s}=\beta_{z^{\prime}, s}$. Hence, $H(\beta, s)=H(\gamma, s)$.

If $r(\gamma)>r(\beta)$, by taking the limit as $s \rightarrow r(\gamma)^{+}$, we deduce that $\gamma=$ $H(\beta, r(\gamma))>H(\beta, r(\beta))=\beta$, a contradiction. Hence, $r(\beta) \geq r(\gamma)$, and by taking the limit as $s \rightarrow r(\beta)^{+}$, we deduce that $\beta=H(\gamma, r(\beta))$, as desired. (For an alternate proof, see [22, Lemma 2.2.12].)

Corollary 2.23. For any $\beta, \gamma \in \mathcal{M}(K[T])$ with $\beta \geq \gamma$, there exist $\tilde{\beta}, \tilde{\gamma} \in$ $\mathcal{M}(\mathbb{C}[T])$ restricting to $\beta, \gamma$, respectively, for which $\tilde{\beta} \geq \tilde{\gamma}$.

Proof. For each finite extension $K^{\prime}$ of $K$, the map $K[T] \rightarrow K^{\prime}[T]$ is split, so by Lemma 1.19 , the restriction map $\mathcal{M}\left(K^{\prime}[T]\right) \rightarrow \mathcal{M}(K[T])$ is surjective. It follows that $\mathcal{M}(\mathbb{C}[T]) \rightarrow \mathcal{M}(K[T])$ is also surjective. (See Remark 2.14 for a more precise statement.) 
We may thus choose $\tilde{\gamma} \in \mathcal{M}(\mathbb{C}[T])$ extending $\gamma$ and then put $\tilde{\beta}=$ $H(\tilde{\gamma}, r(\beta))$. This seminorm restricts to $\beta$ by Theorem 2.11. (For an alternate proof, see [22, Lemma 2.2.9].)

Lemma 2.24. Assume that the norm on $K$ is nontrivial. For $z \in \mathfrak{o}$ and $r \in[0,1]$, let $D(z, r)$ be the set of $\beta_{x, 0} \in \mathcal{M}(K[T])$ for which $\beta_{z, r} \geq \beta_{x, 0}$. Then $D(z, r)=D(z, s)$ if and only if $r=s$.

Proof. It suffices to deduce a contradiction under the assumption that $D(z, r)=D(z, s)$ for some $r>s>0$. Pick $z^{\prime} \in \mathfrak{o}$ integral over $K$ with $\alpha(z-$ $\left.z^{\prime}\right)<s$, so that $D(z, r)=D\left(z^{\prime}, r\right)$ and $D(z, s)=D\left(z^{\prime}, s\right)$. Since $D(z, r)=$ $D(z, s)$, for any $\beta_{x, 0} \in D(z, r)$, we have $\beta_{z^{\prime}, s} \geq \beta_{x, 0}$, and hence (by Lemma 2.19) $\alpha\left(\tau\left(z^{\prime}\right)-x\right) \leq s$ for some $\tau \in \operatorname{Aut}(\mathbb{C} / K)$. Consequently, there are only finitely many points in $\mathcal{M}(\mathbb{C}[T])$ of the form $\tilde{\beta}_{x, s}$ which are dominated by $\tilde{\beta}_{z, r}$.

Pick $u \in \mathfrak{o}$ with $\alpha(u) \in(s, r)$. For $x, x^{\prime} \in \mathfrak{o}$ with $\alpha\left(z^{\prime}-x\right), \alpha\left(z^{\prime}-x^{\prime}\right) \leq$ $\alpha(u)$, declare $x, x^{\prime}$ to be equivalent if $\tilde{\beta}_{x, t}=\tilde{\beta}_{x^{\prime}, t}$ for some $t \in[0, \alpha(u))$. The resulting equivalence classes may be put in bijection with $\kappa_{\mathbb{C}}$ by mapping the class of $x$ to the residue class of $\left(z^{\prime}-x\right) / u$. Since $\kappa_{\mathbb{C}}$ is algebraically closed and hence infinite, this yields the desired contradiction.

Corollary 2.25. Assume that the norm on $K$ is nontrivial. For $z \in \mathfrak{o}$ and $r \in[0,1], \beta_{z, r}=\sup D(z, r)$.

Proof. Put $\gamma_{z, r}=\sup D(z, r)$; it is clear that $\beta_{z, r} \geq \gamma_{z, r}$. By Corollary 2.18, $r\left(\beta_{z, r}\right)=r$. By Theorem 2.11, $\beta_{z, r}=H\left(\gamma_{z, r}, r\right)$.

Suppose that $\beta_{z, r} \neq \gamma_{z, r}$; by Corollary 2.12, $s=r\left(\gamma_{z, r}\right)$ must be strictly less than $r$. Pick $s^{\prime} \in(s, r)$. By Lemma 2.20, we can write $H\left(\gamma_{z, r}, s^{\prime}\right)=$ $\beta_{z^{\prime}, s^{\prime}}$ for some $z^{\prime} \in \mathfrak{o}$ which is integral over $K$. Since $\beta_{z^{\prime}, s^{\prime}} \geq \gamma_{z, r} \geq \beta_{z, 0}$, by Lemma 2.17, $\beta_{z^{\prime}, s^{\prime}}=\beta_{z, s^{\prime}}$.

By Lemma 2.24, we can find $\beta_{z^{\prime \prime}, 0} \in D(z, r)$ with $\beta_{z^{\prime \prime}, 0} \notin D\left(z, s^{\prime}\right)=$ $D\left(z^{\prime}, s^{\prime}\right)$. Hence, $H\left(\gamma_{z, r}, s^{\prime}\right)=\beta_{z^{\prime}, s^{\prime}} \nsupseteq \beta_{z^{\prime \prime}, 0}$, contradicting the fact that $\beta_{z^{\prime}, s^{\prime}} \geq \gamma_{z, r}=\sup D(z, r) \geq \beta_{z^{\prime \prime}, 0}$. This contradiction forces $\beta_{z, r}=\gamma_{z, r}$, as desired.

For completeness, we add a classification result formulated in the style of Berkovich (see Remark 2.9).

THEOREM 2.26. Each element of $\mathcal{M}(K[T])$ is of exactly one of the following four types. 
(i) A point of the form $\beta_{z, 0}$ for some $z \in \mathfrak{o}$. Such a point has radius 0 and is minimal.

(ii) A point of the form $\beta_{z, r}$ for some $z \in \mathfrak{o}$ and some $r \in(0,1]$ which is the norm of an element of $\mathfrak{o}$. Such a point has radius $r$ and is not minimal.

(iii) A point of the form $\beta_{z, r}$ for some $z \in \mathfrak{o}$ and some $r \in(0,1]$ which is not the norm of an element of $\mathfrak{o}$. Such a point has radius $r$ and is not minimal.

(iv) The infimum of a decreasing sequence $\beta_{z_{i}, r_{i}}$ for which the sets $D\left(z_{i}, r_{i}\right)$ have empty intersection. Such a point has radius $\inf _{i}\left\{r_{i}\right\}>0$ and is minimal.

Proof. By Corollary 2.18, $r\left(\beta_{z, r}\right)=r$. Consequently, types (i)-(iii) are mutually exclusive. Moreover, $\beta_{z, r}$ cannot be of type (iv), since $\beta_{z_{i}, r_{i}} \geq \beta_{z, r}$ implies that $\beta_{z, 0} \in D\left(z_{i}, r_{i}\right)$. Consequently, no point can be of more than one type.

It remains to check that any point $\beta \in \mathcal{M}(K[T])$ not of the form $\beta_{z, r}$ is of type (iv) and is minimal of the claimed radius. Choose a sequence $1 \geq r_{1}>r_{2}>\cdots$ with infimum $r(\beta)$. By Lemma 2.20, for each $i$, we have $H\left(\beta, r_{i}\right)=\beta_{z_{i}, r_{i}}$ for some $z_{i} \in \mathfrak{o}$. The sequence $\beta_{z_{1}, r_{1}}, \beta_{z_{2}, r_{2}}, \ldots$ is decreasing with infimum $\beta$; the sequence $D\left(z_{i}, r_{i}\right)$ is also decreasing. For each $z \in \mathfrak{o}$, there exists $i$ for which $\beta_{z, r_{i}} \neq \beta_{z_{i}, r_{i}}$; for such $i$ we have $\beta_{z, 0} \notin D\left(z_{i}, r_{i}\right)$ by Lemma 2.19 . Hence, the $D\left(z_{i}, r_{i}\right)$ have empty intersection; this forces $\inf _{i}\left\{r_{i}\right\}>0$ because $\mathfrak{o}$ is complete. Hence, $\beta$ is of type (iv); it is minimal by Theorem 2.11 plus Lemma 2.20. Since $\beta=\inf _{i}\left\{\beta_{z_{i}, r_{i}}\right\}$ and $r\left(\beta_{z_{i}, r_{i}}\right)=r_{i}$ by Corollary 2.18 , we have $r(\beta) \geq \inf _{i}\left\{r_{i}\right\}$; the reverse inequality also holds because $r_{i}=r\left(\beta_{z_{i}, r_{i}}\right) \geq r(\beta)$ by Corollary 2.12 .

This classification can be used to describe the residual extensions and norm groups of points in $\mathcal{M}(K[T])$. (For similar results, see $[22$, Lemma 2.2.18] or [27, Section 3].)

Corollary 2.27. Let $\beta$ be a point of $\mathcal{M}(K[T])$, classified according to Theorem 2.26. Let $\left|\alpha^{\times}\right|,\left|\beta^{\times}\right|$denote the groups of nonzero values assumed by $\alpha, \beta$, respectively.

(i) For $\beta$ of type (i), $\kappa_{\mathcal{H}(\beta)}$ is algebraic over $\kappa_{K}$, and $\left|\beta^{\times}\right| /\left|\alpha^{\times}\right|$is a torsion group.

(ii) For $\beta$ of type (ii), $\kappa_{\mathcal{H}(\beta)}$ is finitely generated over $\kappa_{K}$ of transcendence degree 1 , and $\left|\beta^{\times}\right| /\left|\alpha^{\times}\right|$is a finite group. 
(iii) For $\beta$ of type (iii), $\kappa_{\mathcal{H}(\beta)}$ is a finite extension of $\kappa_{K}$, and $\left|\beta^{\times}\right| /\left|\alpha^{\times}\right|$is a finitely generated abelian group of rank 1 .

(iv) For $\beta$ of type (iv), $\kappa_{\mathcal{H}(\beta)}$ is algebraic over $\kappa_{K}$, and $\left|\beta^{\times}\right| /\left|\alpha^{\times}\right|$is a torsion group.

Proof. Recall that for $L / K$ a finite extension of complete nonarchimedean fields, $\kappa_{L}$ is a finite extension of $\kappa_{K}$ and $\left|L^{\times}\right| /\left|K^{\times}\right|$is a finite group. More precisely, by a theorem of Ostrowski (see [25, Theorem 6.2]),

$$
\frac{[L: K]}{\left[\kappa_{L}: \kappa_{K}\right] \#\left(\left|L^{\times}\right| /\left|K^{\times}\right|\right)} \begin{cases}=1 & \left(\operatorname{char}\left(\kappa_{K}\right)=0\right), \\ \in\left\{1, p, p^{2}, \ldots\right\} & \left(\operatorname{char}\left(\kappa_{K}\right)=p>0\right) .\end{cases}
$$

Consequently, in cases (ii) and (iii), it is enough to check the claims after replacing $K$ by a finite extension; in cases (i) and (iv), we may replace $K$ by $\mathbb{C}$ itself. We make these assumptions hereafter.

In cases (i)-(iii), we may now assume that $\beta=\beta_{z, r}$ with $z \in \mathfrak{o}_{K}$. In case $(\mathrm{i}), \mathcal{H}(\beta)=K$; in case (ii), $\kappa_{\mathcal{H}(\beta)} \cong \kappa_{K}(x)$ for $x$ the class of $(T-z) / u$ for any $u \in K$ of norm $r$, and $\left|\beta^{\times}\right| /\left|\alpha^{\times}\right|$is trivial; in case (iii), $\kappa_{\mathcal{H}(\beta)}=\kappa_{K}$ and $\left|\beta^{\times}\right| /\left|\alpha^{\times}\right|$is free on the generator $r$.

In case (iv), the norm $\alpha$ must be nontrivial. By Corollary 2.21, for each $y \in K[T]$, any sufficiently small $s \in(r(\beta), 1]$ satisfies $H(\beta, s)(y)=\beta(y)$. If we choose $s \in\left|\alpha^{\times}\right|$, we deduce that $\left|\beta^{\times}\right| /\left|\alpha^{\times}\right|$is trivial. If we choose $s \notin\left|\alpha^{\times}\right|$, then for any $z \in K[T]$ with $\beta(z) \leq \beta(y)$, by case (iii), there must exist $\lambda \in K$ for which $H(\beta, s)(z-\lambda y)<H(\beta, s)(y)$. This implies that

$$
\beta(z-\lambda y) \leq H(\beta, s)(z-\lambda y)<H(\beta, s)(y)=\beta(y),
$$

so $z / y$ and $\lambda$ have the same image in $\kappa_{\mathcal{H}(\beta)}$. Hence, $\kappa_{\mathcal{H}(\beta)}=\kappa_{K}$.

REMARK 2.28. In (i) and (iv) of Corollary 2.27, it is not guaranteed that $\kappa_{\mathcal{H}(\beta)}$ is finite over $\kappa_{K}$ or that $\left|\beta^{\times}\right| /\left|\alpha^{\times}\right|$is a finite group. We illustrate this with an example of a point of type (i) for which $\left|\beta^{\times}\right| /\left|\alpha^{\times}\right|$is infinite; the other claims can be seen by similar arguments.

Let $F$ be an algebraically closed field of characteristic 0 , and take $K=$ $F((U))$ equipped with the $U$-adic norm (for any normalization). We may then identify $\mathbb{C}$ with the completion of the field of Puiseux series in $U$ over $F$. Inside $\mathbb{C}$, take $z=\sum_{n=1}^{\infty} U^{n+1 / n !}$, and put $\beta=\beta_{z, 0} \in \mathcal{M}(K[T])$. We may establish by induction that for each positive integer $m,|U|^{1 / m !} \in$ $\left|\beta^{\times}\right|$: this is apparent for $m=1$, and given that this holds for $m-1$, we have $F\left(\left(U^{1 /(m-1) !}\right)\right) \subseteq \mathcal{H}(\beta)$ and $\beta\left(T-\sum_{n=1}^{m-1} U^{n+1 / n !}\right)=|U|^{m+1 / m !}$. Consequently, $\left|\beta^{\times}\right| /\left|\alpha^{\times}\right| \cong \mathbb{Q} / \mathbb{Z}$ is not finite. 
REMARK 2.29. Theorem 2.11 implies that as a partially ordered set under domination, $\mathcal{M}(K[T])$ carries the structure of a tree. One can capture the tree structure in other ways, for instance, by exhibiting $\mathcal{M}(K[T])$ as an inverse limit of finite contractible simplicial complexes (see, e.g., [1, Proposition 1.19]). (This construction is the simplest meaningful case of the main result of [24].)

The geometry of $\mathcal{M}(K[T])$, including the tree interpretation, has been deployed in a number of apparently unrelated fields. Here are some representative (but not exhaustive) examples.

- Favre and Jonsson ([12], [14], [13]) use the tree structure to study plurisubharmonic singularities of functions of two complex variables. Some progress has been made in extending to more variables by Boucksom, Favre, and Jonsson [8].

- Kedlaya ([18], [21]) uses the tree structure to study the local structure of irregular flat meromorphic connections on algebraic and algebraic varieties. A related development in $p$-adic cohomology is [22].

- Temkin [27] uses the tree structure to establish local uniformization in positive characteristic up to an inseparable morphism.

- Numerous applications have been found in the theory of dynamical systems. A good starting point for this line of inquiry is Baker and Rumely [1].

- A development closely related to the previous one is the use of nonarchimedean potential theory in Arakelov theory, for example, in the study of equidistribution questions. This is pursued thoroughly in the work of Chambert-Loir and his collaborators (see, e.g., [10]).

\section{$\S 3$. Witt vectors}

We now introduce the ring of Witt vectors over a perfect ring of characteristic $p$. These behave a bit like power series in the variable $p$ with coefficients in the given ring, with the role of the structure morphism (the injection of the coefficient ring into the series ring) played by the Teichmüller map. The latter map is multiplicative but not additive; nonetheless, we can use it to define raising and lowering operators $\lambda, \mu$ analogous to the ones from Section 2. (We previously considered these operators in [20].)

Hypothesis 3.1. For the remainder of the paper, let $R$ denote an $\mathbb{F}_{p^{-}}$ algebra which is perfect, that is, for which the pth power map is a bijection. Unless otherwise specified, equip $R$ with the trivial norm. 
REMARK 3.2. If $R$ is an $\mathbb{F}_{p}$-algebra which is not necessarily perfect, we can form the perfect closure $R^{\text {perf }}$ as the limit of the direct system $R \rightarrow R \rightarrow \cdots$ in which each arrow is the $p$ th power map. We obtain a natural map $R \rightarrow$ $R^{\text {perf }}$ by mapping to the initial term of the direct system; the corresponding map $\mathcal{M}\left(R^{\text {perf }}\right) \rightarrow \mathcal{M}(R)$ is easily seen to be a homeomorphism.

Definition 3.3. A strict p-ring is a (commutative unital) ring $S$ which is $p$-torsion-free and $p$-adically complete and separated, and for which $S / p S$ is a perfect $\mathbb{F}_{p}$-algebra.

LEMMA 3.4. Let $S$ be a strict $p$-ring with $S / p S \cong R$.

(a) Given $\bar{x} \in R$, let $x_{n} \in S$ be any lift of $\bar{x}^{p^{-n}}$. Then the sequence $x_{n}^{p^{n}}$ converges $p$-adically to a limit $[\bar{x}]$ (the Teichmüller lift of $\bar{x}$ ), which is the unique lift of $\bar{x}$ admitting a $p^{n}$ th root in $S$ for each nonnegative integer $n$.

(b) The resulting Teichmüller map [·] $R \rightarrow S$ is multiplicative.

(c) Each $x \in S$ admits a unique representation $\sum_{i=0}^{\infty} p^{i}\left[\overline{x_{i}}\right]$ with $\overline{x_{i}} \in R$.

Proof. By the binomial theorem, $a \equiv b\left(\bmod p^{m}\right)$ implies that $a^{p} \equiv b^{p}$ $\left(\bmod p^{m+1}\right)$. Consequently,

$$
x_{m+1}^{p^{m+1}} \cong x_{m}^{p^{m}} \quad\left(\bmod p^{m+1}\right)
$$

so the $x_{m}^{p^{m}}$ converge to a limit $[\bar{x}]$. Similarly, for each nonnegative integer $n$, the $x_{m+n}^{p^{m}}$ converge to a $p^{n}$ th root of $[\bar{x}]$. If $x^{\prime}$ is another lift of $\bar{x}$ admitting a $p^{n}$ th root $x_{n}^{\prime}$ for each nonnegative integer $n$, then

$$
x_{m}^{p^{m}} \cong\left(x_{m}^{\prime}\right)^{p^{m}}=x^{\prime} \quad\left(\bmod p^{m+1}\right),
$$

so $[\bar{x}]=x^{\prime}$. This proves $(\mathrm{a})$.

Given (a), the product of two Teichmüller lifts admits a $p^{n}$ th root for each nonnegative integer $n$ and so must also be a Teichmüller lift; this yields (b). Since $[\bar{x}]$ is always a lift of $\bar{x},(c)$ follows (see also [26, Section II.4, Proposition 8]).

THEOREM 3.5. There exists a unique (up to unique isomorphism) strict $p$-ring $W(R)$ for which $W(R) /(p) \cong R$. Moreover, the correspondence $R \rightsquigarrow$ $W(R)$ is covariantly functorial in $R$.

Sketch of proof. For $n=0,1, \ldots$, put

$$
W_{n}\left(X_{0}, \ldots, X_{n}\right)=\sum_{i=0}^{n} p^{i} X_{i}^{p^{n-i}}
$$


Given that $\Phi \in \mathbb{Z}[X, Y]$, there exists a unique sequence $\phi_{0}, \phi_{1}, \ldots$ with $\phi_{n} \in$ $\mathbb{Z}\left[X_{0}, \ldots, X_{n}, Y_{0}, \ldots, Y_{n}\right]$ such that

$$
\begin{aligned}
& W_{n}\left(\phi_{0}, \ldots, \phi_{n}\right)=\Phi\left(W_{n}\left(X_{0}, \ldots, X_{n}\right), W_{n}\left(Y_{0}, \ldots, Y_{n}\right)\right) \\
& \quad(n=0,1, \ldots)
\end{aligned}
$$

using the sequences associated to the polynomials $X-Y, X Y$, we define subtraction and multiplication rules on the set of sequences $\overline{x_{0}}, \overline{x_{1}}, \ldots$ with values in $R$. This yields a strict $p$-ring $W(R)$ with $W(R) /(p) \cong R$; more precisely, the sequence $\overline{x_{0}}, \overline{x_{1}}, \ldots$ corresponds to the element $\sum_{i=0}^{\infty} p^{i}\left[{\overline{x_{i}}}^{p^{-i}}\right]$. (See [26, Section II.6] for further details.)

Definition 3.6. The ring $W(R)$ of Theorem 3.5 is called the ring of p-typical Witt vectors with coefficients in $R$; unless otherwise specified, we equip $W(R)$ with the $p$-adic norm normalized with $|p|=p^{-1}$. Since its construction is functorial in $R, W(R)$ carries an automorphism $\phi$ which corresponds to (and lifts) the $p$-power Frobenius map on $R$, called the Witt vector Frobenius.

REMARK 3.7. The addition and multiplication of general elements of $W(R)$ are somewhat complicated to express explicitly. One important consequence of (3.5.1) is that if we write $x=\sum_{i=0}^{\infty} p^{i}\left[\overline{x_{i}}\right], y=\sum_{i=0}^{\infty} p^{i}\left[\overline{y_{i}}\right], x-y=$

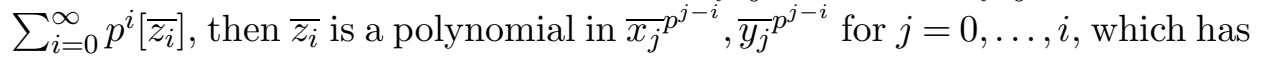
integer coefficients, is homogeneous of degree 1 for the weighting in which $\overline{x_{j}}, \overline{y_{j}}$ have degree 1 , and belongs to the ideal generated by $\overline{x_{j}} p^{j-i}-\bar{y}_{j} p^{j-i}$ for $j=0, \ldots, i$ (because it vanishes whenever $x=y$ ). (See also Lemma 3.8 below.)

Lemma 3.8. For $\bar{x} \in R$, write $[\bar{x}+1]-1=\sum_{i=0}^{\infty} p^{i}\left[P_{i}\left(\bar{x}^{p^{-i}}\right)\right]$ with $P_{i}(T) \in$ $\mathbb{F}_{p}[T]$ as in Remark 3.7. Then $P_{i}(T) \equiv T\left(\bmod T^{2}\right)$.

Proof. Since $[\bar{x}+1]-1$ vanishes when $\bar{x}=0$, the polynomial $P_{i}(T)$ is divisible by $T$. To obtain the congruence modulo $T^{2}$, note that

$$
P_{i}(T) \equiv p^{-i}\left((T+1)^{p^{i}}-1-\sum_{j=0}^{i-1} p^{j} P_{j}(T)^{p^{i-j}}\right) \quad(\bmod p) .
$$

The coefficient of $T$ on the right-hand side equals 1 (from $p^{-i}(T+1)^{p^{i}}$ ) plus a multiple of $p$ (from all other terms). This proves the claim. 
REMARK 3.9. Suppose that $\overline{x_{1}}, \ldots, \overline{x_{n}}$ generate the unit ideal in $R$. Then $\left[\overline{x_{1}}\right], \ldots,\left[\overline{x_{n}}\right]$ generate an ideal in $W(R)$ containing an element congruent to 1 modulo $p$. However, any such element is a unit, so the ideal generated is the unit ideal.

There are two different meaningful types of polynomial extensions of a Witt ring $W(R)$ : the usual polynomial extension of the ring $W(R)$ itself, and the Witt ring of the perfection of the polynomial extension of the base rings. These rings enjoy the following relationship.

LEMma 3.10. Equip $W(R)[T]$ with the Gauss extension of the p-adic norm.

(a) The isometric homomorphism $\psi: W(R)[T] \rightarrow W\left(R[\bar{T}]^{\text {perf }}\right)$ which maps $W(R)$ to $W\left(R[\bar{T}]^{\text {perf }}\right)$ via the functoriality of Witt vectors, and which sends $T$ to $[\bar{T}]$, is split.

(b) The map $\psi^{*}$ is a quotient map of topological spaces.

Proof. Via $\psi$, we may identify $W\left(R[\bar{T}]^{\text {perf }}\right)$ with the $p$-adic completion of $\bigcup_{n=1}^{\infty} W(R)\left[T^{p^{-n}}\right]$. Under this identification, we obtain a splitting by omitting all nonintegral powers of $T$. Hence, $\psi$ is split, yielding (a).

Since $\psi$ is split, $\psi^{*}$ is surjective by Lemma 1.19. Let $U \subseteq \mathcal{M}(W(R)[T])$ be a subset whose inverse image $V$ in $\mathcal{M}\left(W\left(R[\bar{T}]^{\text {perf }}\right)\right)$ is open. Let $U^{\prime}, V^{\prime}$ be the complements of $U, V$, respectively. Then $V^{\prime}$ is closed and hence compact because $\mathcal{M}\left(W\left(R[\bar{T}]^{\text {perf }}\right)\right)$ is compact. Since $U^{\prime}=\psi^{*}\left(V^{\prime}\right), U^{\prime}$ is quasicompact and hence closed because $\mathcal{M}(W(R)[T])$ is Hausdorff. Hence, $U$ is open; this proves that $\psi^{*}$ is a quotient map, yielding (b).

Remark 3.11. Define the map $\delta: W(R) \rightarrow W(R)$ by the formula

$$
\delta(s)=p^{-1}\left(\phi(s)-s^{p}\right) \quad(s \in W(R)) .
$$

The map $\delta$ is an example of a $p$-derivation on $W(R)$, in that it has the following properties.

(a) We have $\delta(1)=0$. (In this example, we also have $\delta([r])=0$ for all $r \in R$.)

(b) For all $s_{1}, s_{2} \in W(R), \delta\left(s_{1}+s_{2}\right)=\delta\left(s_{1}\right)+\delta\left(s_{2}\right)-P\left(s_{1}, s_{2}\right)$, where the polynomial $P(X, Y) \in \mathbb{Z}[X, Y]$ is given by $P(X, Y)=p^{-1}\left((X+Y)^{p}-\right.$ $\left.X^{p}-Y^{p}\right)$.

(c) For all $s_{1}, s_{2} \in W(R), \delta\left(s_{1} s_{2}\right)=s_{1}^{p} \delta\left(s_{2}\right)+s_{2}^{p} \delta\left(s_{1}\right)+p \delta\left(s_{1}\right) \delta\left(s_{2}\right)$.

Such maps were introduced (with a slightly different sign convention) by Joyal [16] and later exploited heavily by Buium [9] to transfer some concepts 
from the theory of differential equations into arithmetic geometry. We had hoped to use the $p$-derivation $\delta$ to construct an analogue of the formula (2.3.1), but so far we have not found any such analogue. We will thus be forced to work more indirectly (see Definition 5.2 and Theorem 5.11).

\section{$\S 4$. Raising and lowering for Witt vectors}

The raising and lowering operators for Witt vectors are defined as follows.

Lemma 4.1. For $\alpha$ a submultiplicative (resp., power-multiplicative, multiplicative) seminorm on $R$ bounded by the trivial norm, the function $\lambda(\alpha)$ : $W(R) \rightarrow[0,1]$ given by

$$
\lambda(\alpha)\left(\sum_{i=0}^{\infty} p^{i}\left[{\overline{x_{i}}}^{p^{-i}}\right]\right)=\max _{i}\left\{p^{-i} \alpha\left(\overline{x_{i}}\right)^{p^{-i}}\right\}
$$

is a submultiplicative (resp., power-multiplicative, multiplicative) seminorm on $W(R)$ bounded by the p-adic norm.

We will mostly apply this result when $\alpha$ is power-multiplicative, in which case we may use the simpler formula

$$
\lambda(\alpha)\left(\sum_{i=0}^{\infty} p^{i}\left[\overline{x_{i}}\right]\right)=\max _{i}\left\{p^{-i} \alpha\left(\overline{x_{i}}\right)\right\} .
$$

Proof. For $\bar{x}, \bar{y} \in R$ and $i$ a nonnegative integer, write $[\bar{x}]-[\bar{y}]=$ $\sum_{j=0}^{\infty} p^{j}\left[\overline{z_{j}} p^{-j}\right]$. By Remark 3.7, $\overline{z_{j}}$ is a polynomial in $\bar{x}, \bar{y}$ which has integer coefficients and is homogeneous of degree $p^{j}$. This remains true after taking $p^{i}$ th powers, so we may deduce that $\alpha\left(\overline{z_{j}} p^{i}\right) \leq \max \left\{\alpha\left(\bar{x}^{p^{i}}\right)^{p^{j}}, \alpha\left(\bar{y}^{p^{i}}\right)^{p^{j}}\right\}$. Consequently,

$$
\begin{aligned}
& \lambda(\alpha)\left(p^{i}([\bar{x}]-[\bar{y}])\right) \\
& \quad=\lambda(\alpha)\left(\sum_{j=0}^{\infty} p^{i+j}\left[\left({\overline{z_{j}}}^{p^{i}}\right) p^{-i-j}\right]\right)=\max _{j}\left\{p^{-i-j} \alpha\left({\overline{z_{i}}}^{p^{i}}\right)^{p^{-i-j}}\right\} \\
& \quad \leq p^{-i} \max \left\{\alpha\left(\bar{x}^{p^{i}}\right)^{p^{-i}}, \alpha\left(\bar{y}^{p^{i}}\right)^{p^{-i}}\right\}=\max \left\{\lambda(\alpha)\left(p^{i}[\bar{x}]\right), \lambda(\alpha)\left(p^{i}[\bar{y}]\right)\right\} .
\end{aligned}
$$

Similarly, $\lambda(\alpha)\left(p^{i}([\bar{x}]+[\bar{y}])\right) \leq \max \left\{\lambda(\alpha)\left(p^{i}[\bar{x}]\right), \lambda(\alpha)\left(p^{i}[\bar{y}]\right)\right\}$.

We next establish that $\lambda(\alpha)$ is a seminorm. Let $x=\sum_{i=0}^{\infty} p^{i}\left[\overline{x_{i}}\right], y=$ $\sum_{i=0}^{\infty} p^{i}\left[\overline{y_{i}}\right]$ be two general elements of $W(R)$, and write $x-y=\sum_{i=0}^{\infty} p^{i}\left[\overline{z_{i}}\right]$. 
For each nonnegative integer $n$, we will exhibit an equality of the form

$$
x-y=\left(\sum_{i=0}^{n-1} p^{i}\left[\bar{z}_{i}\right]\right) \pm p^{n} w_{1} \pm \cdots \pm p^{n} w_{k}
$$

for some nonnegative integer $k=k(n)$ and some $w_{1}, \ldots, w_{k} \in W(R)$ with the property that

$$
\begin{aligned}
& \lambda(\alpha)\left(\sum_{i=0}^{n-1} p^{i}\left[\bar{z}_{i}\right]\right), \lambda(\alpha)\left(p^{n} w_{1}\right), \ldots, \lambda(\alpha)\left(p^{n} w_{k}\right) \\
& \quad \leq \max \{\lambda(\alpha)(x), \lambda(\alpha)(y)\} .
\end{aligned}
$$

From this, it will follow at once that $\lambda(\alpha)(x-y) \leq \max \{\lambda(\alpha)(x), \lambda(\alpha)(y)\}$ and hence that $\lambda(\alpha)$ is a seminorm.

Suppose that for some nonnegative integer $n$, we are given $w_{1}, \ldots, w_{k}$ satisfying (4.1.1) and (4.1.2). Then condition (4.1.2) is preserved by modifying (4.1.1) in the following ways.

(1) Given a term $\pm p^{n} w_{j}$ in (4.1.1), replace it with the sum of two terms, one of which is $\pm p^{n}$ times a Teichmüller element.

(2) Given two terms of the form $\pm p^{n}[\bar{w}]$ and $\pm p^{n}\left[\bar{w}^{\prime}\right]$, replace them with their sum. This maintains (4.1.2) by our earlier argument.

Moreover, the number of summands in (4.1.1) among $\pm p^{n} w_{1}, \ldots, \pm p^{n} w_{k}$ which are not divisible by $p^{n+1}$ never increases and, in fact, always decreases in step (2) unless one of the two terms is itself divisible by $p^{n+1}$. Consequently, using these operations, we can always arrive at the situation where one of the summands in (4.1.1) among $\pm p^{n} w_{1}, \ldots, \pm p^{n} w_{k}$ equals $p^{n}\left[\bar{z}_{n}\right]$ and the others are divisible by $p^{n+1}$. This yields a sum of the desired form with $n$ replaced by $n+1$, completing the proof that $\lambda(\alpha)$ is a seminorm. This in turn implies that

$$
\lambda(\alpha)(x y) \leq \max _{i, j}\left\{\lambda(\alpha)\left(p^{i}\left[\overline{x_{i}}\right] p^{j}\left[\overline{y_{j}}\right]\right)\right\} \leq \lambda(\alpha)(x) \lambda(\alpha)(y),
$$

so $\lambda(\alpha)$ is submultiplicative.

Suppose now that $\alpha$ is multiplicative. To check that $\lambda(\alpha)$ is multiplicative, it is enough to check that $\lambda(\alpha)(x y) \geq \lambda(\alpha)(x) \lambda(\alpha)(y)$ in case $\lambda(\alpha)(x)$, $\lambda(\alpha)(y)>0$. Choose the minimal indices $j, k$ for which $\lambda(\alpha)\left(p^{j}\left[\overline{x_{j}}\right]\right)$, $\lambda(\alpha)\left(p^{k}\left[\overline{y_{k}}\right]\right)$ attain their maximal values. For

$$
x^{\prime}=\sum_{i=j}^{\infty} p^{i}\left[\overline{x_{i}}\right], \quad y^{\prime}=\sum_{i=k}^{\infty} p^{i}\left[\overline{y_{i}}\right],
$$


on one hand, we may write $x^{\prime} y^{\prime}=\sum_{i=j+k}^{\infty} p^{i}\left[\overline{z_{i}}\right]$ with $\overline{z_{j+k}}=\overline{x_{j} y_{k}}$. By submultiplicativity, $\quad \lambda(\alpha)\left(x^{\prime} y^{\prime}\right) \geq \lambda(\alpha)(x) \lambda(\alpha)(y) \geq \lambda(\alpha)\left(x^{\prime}\right) \lambda(\alpha)\left(y^{\prime}\right) \geq$ $\lambda(\alpha)\left(x^{\prime} y^{\prime}\right)$, so $\lambda(\alpha)\left(x^{\prime} y^{\prime}\right)=\lambda(\alpha)(x) \lambda(\alpha)(y)$. On the other hand, we have $\lambda(\alpha)\left(x-x^{\prime}\right)<\lambda(\alpha)(x), \quad \lambda(\alpha)\left(y-y^{\prime}\right)<\lambda(\alpha)(y)$, so $\lambda(\alpha)\left(x y-x^{\prime} y^{\prime}\right)<$ $\lambda(\alpha)(x) \lambda(\alpha)(y)=\lambda(\alpha)\left(x^{\prime} y^{\prime}\right)$. Putting everything together, we deduce that $\lambda(\alpha)$ is multiplicative. Similarly, if $\alpha$ is power-multiplicative, we see that $\lambda(\alpha)$ is power-multiplicative by taking $x=y$ in the preceding argument.

Corollary 4.2. For $\alpha$ a submultiplicative (resp., power-multiplicative, multiplicative) seminorm on $R$ bounded by the trivial norm, the function $\Lambda(\alpha): W(R) \rightarrow[0,1]$ given by

$$
\Lambda(\alpha)\left(\sum_{i=0}^{\infty} p^{i}\left[{\overline{x_{i}}}^{p^{-i}}\right]\right)=\sup _{i}\left\{\alpha\left(\overline{x_{i}}\right)^{p^{-i}}\right\}
$$

is a submultiplicative (resp., power-multiplicative, multiplicative) seminorm on $W(R)$ bounded by the trivial norm.

Proof. For $x \in W(R)$, we have

$$
\Lambda(\alpha)(x)=\lim _{c \rightarrow+\infty} \lambda\left(\alpha^{c}\right)(x)^{1 / c} .
$$

The claims then follow from Lemma 4.1.

REMARK 4.3. Although $\Lambda(\alpha)$ may seem like a more natural analogue of the Gauss extension than $\lambda(\alpha)$, the proof of the continuity of $\lambda$ (Theorem 4.5) does not apply to $\Lambda$ (see Remark 4.6). We thus work primarily with $\lambda$ hereafter.

LEMMA 4.4. For $\beta$ a power-multiplicative (resp., multiplicative) seminorm on $W(R)$ bounded by the $p$-adic norm, the function $\mu(\beta): R \rightarrow[0,1]$ given by

$$
\mu(\beta)(\bar{x})=\beta([\bar{x}])
$$

is a power-multiplicative (resp., multiplicative) seminorm bounded by the trivial norm.

Proof. Given $\bar{x}, \bar{y} \in R$, choose any $x, y \in W(R)$ lifting them. For $(\bar{z}, z)=$ $(\bar{x}, x),(\bar{y}, y),(\bar{x}+\bar{y}, x+y)$, for any $\epsilon>0$, for $n$ sufficiently large, we have

$$
\max \{\epsilon, \mu(\beta)(\bar{z})\}=\max \left\{\epsilon, \beta\left(\phi^{-n}(z)^{p^{n}}\right)\right\}
$$


because $\phi^{-n}\left(z^{p^{n}}\right)$ converges $p$-adically to $[\bar{z}]$ by Lemma 3.4(a). Since $\beta$ is a power-multiplicative (resp., multiplicative) seminorm, we deduce that $\mu(\beta)$ is one as well. (An alternate proof can be obtained using Remark 3.7.)

We now have the following analogue of Theorem 2.2.

Theorem 4.5. Equip $R$ with the trivial norm, and equip $W(R)$ with the p-adic norm. Define $\lambda: \mathcal{M}(R) \rightarrow \mathcal{M}(W(R)), \mu: \mathcal{M}(W(R)) \rightarrow \mathcal{M}(R)$ as in Lemmas 4.1 and 4.4 .

(a) The functions $\lambda, \mu$ are strongly continuous and monotonic.

(b) For all $\alpha \in \mathcal{M}(R),(\mu \circ \lambda)(\alpha)=\alpha$.

(c) For all $\beta \in \mathcal{M}(W(R)),(\lambda \circ \mu)(\beta) \geq \beta$.

Proof. For $x=\sum_{i=0}^{\infty} p^{i}\left[\overline{x_{i}}\right] \in W(R)$ and $\epsilon>0$, choose $j>0$ for which $p^{-j}<\epsilon$; then $\lambda(\alpha)\left(p^{i}\left[\overline{x_{i}}\right]\right)<\epsilon$ for all $\alpha \in \mathcal{M}(R)$ and all $i \geq j$. We thus have

$$
\begin{aligned}
& \{\alpha \in \mathcal{M}(R): \lambda(\alpha)(x)>\epsilon\}=\bigcup_{i=0}^{j-1}\left\{\alpha \in \mathcal{M}(R): \alpha\left(\overline{x_{i}}\right)>p^{i} \epsilon\right\}, \\
& \{\alpha \in \mathcal{M}(R): \lambda(\alpha)(x)<\epsilon\}=\bigcap_{i=0}^{j-1}\left\{\alpha \in \mathcal{M}(R): \alpha\left(\overline{x_{i}}\right)<p^{i} \epsilon\right\},
\end{aligned}
$$

so $\lambda$ is continuous. Similarly, the inverse image of a Weierstrass (resp., Laurent) subspace of $\mathcal{M}(W(R))$ is a finite union of Weierstrass (resp., Laurent) subspaces of $\mathcal{M}(R)$. Now let

$$
U=\left\{\beta \in \mathcal{M}(W(R)): \beta\left(x_{i}\right) \leq q_{i} \beta(y)(i=1, \ldots, n)\right\}
$$

be a rational subspace of $\mathcal{M}(W(R))$ for some $x_{1}, \ldots, x_{n}, y \in W(R)$ generating the unit ideal and some $q_{1}, \ldots, q_{n}>0$. Write $x_{i}=\sum_{j=0}^{\infty} p^{j}\left[\overline{x_{i j}}\right]$ and $y=\sum_{j=0}^{\infty} p^{j}\left[\overline{y_{j}}\right]$; then the $\overline{x_{i j}}$ and $\overline{y_{j}}$ together must generate the unit ideal (in fact, only the $\overline{x_{i 0}}$ and $\overline{y_{0}}$ are needed). Moreover, by Remark 1.15, we can choose some nonnegative integer $m$ so that for $x_{i}^{\prime}=\sum_{j=0}^{m} p^{j}\left[\overline{x_{i j}}\right]$ and $y^{\prime}=\sum_{j=0}^{m} p^{j}\left[\overline{y_{j}}\right]$, we also have

$$
U=\left\{\beta \in \mathcal{M}(W(R)): \beta\left(x_{i}^{\prime}\right) \leq q_{i} \beta\left(y^{\prime}\right)(i=1, \ldots, n)\right\} .
$$

We may then write 


$$
\begin{aligned}
\lambda^{-1}(U)= & \left\{\alpha \in \mathcal{M}(R): \max _{i, j: j \leq m}\left\{p^{-j} \alpha\left(\overline{x_{i j}}\right) / q_{i}\right\} \leq \max _{j \leq m}\left\{p^{-j} \alpha\left(\overline{y_{j}}\right)\right\}\right\} \\
= & \bigcup_{l=0}^{m}\left\{\alpha \in \mathcal{M}(R): p^{-j} \alpha\left(\overline{x_{i j}}\right) \leq q_{i} p^{-l} \alpha\left(\overline{y_{l}}\right), p^{-j} \alpha\left(\overline{y_{j}}\right) \leq p^{-l} \alpha\left(\overline{y_{l}}\right)\right. \\
& (i=1, \ldots, n ; j=0, \ldots, m)\},
\end{aligned}
$$

which is a finite union of rational subspaces of $\mathcal{M}(R)$. Hence, $\lambda$ is strongly continuous.

For $\bar{x} \in R$ and $\epsilon>0$, we have

$$
\begin{aligned}
& \{\beta \in \mathcal{M}(W(R)): \mu(\beta)(\bar{x})>\epsilon\}=\{\beta \in \mathcal{M}(W(R)): \beta([\bar{x}])>\epsilon\}, \\
& \{\beta \in \mathcal{M}(W(R)): \mu(\beta)(\bar{x})<\epsilon\}=\{\beta \in \mathcal{M}(W(R)): \beta([\bar{x}])<\epsilon\},
\end{aligned}
$$

so $\mu$ is continuous. Similarly, the inverse image of a Weierstrass (resp., Laurent, rational) subspace of $\mathcal{M}(W(R))$ is a Weierstrass (resp., Laurent, rational) subspace of $\mathcal{M}(R)$, using Remark 3.9 in the rational case. Since monotonicity is evident, this yields (a).

The equality (b) is evident from the definitions. The inequality (c) follows from the definition of $\lambda$ and the observation that $(\lambda \circ \mu)(\beta)\left(p^{n}[\bar{x}]\right) \geq \beta\left(p^{n}[\bar{x}]\right)$ for any $\bar{x} \in R$ and any nonnegative integer $n$.

REMARK 4.6. The proof of continuity of $\lambda$ in Theorem 4.5 does not apply to $\Lambda$, because we cannot avoid writing $\{\alpha \in \mathcal{M}(R): \Lambda(\alpha)(x)<\epsilon\}$ as an infinite intersection of open sets. Similarly, we make no statement (beyond closure) concerning the inverse image under $\lambda$ of a subspace of $\mathcal{M}(W(R))$ of the form $\{\beta \in \mathcal{M}(W(R)): \beta(x)=0\}$, because the inverse image is defined by the vanishing of infinitely many elements of $R$.

EXAMPLE 4.7. Here is a simple example to illustrate that $\lambda \circ \mu$ need not be the identity map. Put $R=\mathbb{F}_{p}[X]^{\text {perf }}$, so that $W(R)$ is isomorphic to the $p$-adic completion of $\bigcup_{n=1}^{\infty} \mathbb{Z}_{p}\left[[\bar{X}]^{p^{-n}}\right]$ (compare Lemma 3.10). The ring $W(R) /([\bar{X}]-p)$ is isomorphic to the completion of $\bigcup_{n=1}^{\infty} \mathbb{Z}_{p}\left[p^{p^{-n}}\right]$ for the unique multiplicative extension of the $p$-adic norm; let $\beta \in \mathcal{M}(W(R))$ be the induced seminorm.

Note that $\mu(\beta)(\bar{X})=\beta([\bar{X}])=p^{-1}$ and that $\mu(\beta)(y)=1$ for $y \in \mathbb{F}_{p}^{\times}$. These imply that $\mu(\beta)(y) \leq p^{-p^{-n}}$ whenever $y \in \mathbb{F}_{p}\left[\bar{X}^{p^{-n}}\right]$ is divisible by $\bar{X}^{p^{-n}}$, so $\mu(\beta)(y)=1$ whenever $y \in \mathbb{F}_{p}^{\times}+\bar{X}^{p^{-n}} \mathbb{F}_{p}\left[\bar{X}^{p^{-n}}\right]$. We conclude that $\mu(\beta)$ equals the $\bar{X}$-adic norm on $R$ with the normalization $\mu(\beta)(\bar{X})=p^{-1}$. In particular, we have a strict inequality $(\lambda \circ \mu)(\beta)>\beta$. 
REMARK 4.8. The corresponding results from [20, Section 2] are stated with $\mathcal{M}(W(R))$ replaced by the slightly smaller space $\mathcal{M}\left(W(R)\left[p^{-1}\right]\right)$, with the arguments unchanged. Note, however, that extending $\lambda(\alpha)$ to $W(R)\left[p^{-1}\right]$ requires that $\lambda(\alpha)(p x)=p^{-1} \lambda(\alpha)(x)$, and this holds only if $\alpha$ is powermultiplicative. We will thus mostly restrict to this case in what follows. This is no serious loss for our purposes, because replacing a seminorm on $R$ with its spectral seminorm does not change the spectrum.

\section{$\S 5$. Gauss norms}

For $\alpha$ a submultiplicative seminorm on $R$ bounded by the trivial norm, the submultiplicative seminorm $\lambda(\alpha)$ on $W(R)$ behaves like a $\left(p^{-1}\right)$-Gauss seminorm for the generator $p$. One would like analogues of Gauss seminorms for other generators, but unlike in the polynomial case, these cannot be constructed by using automorphisms of $W(R)$ to move generators around. Instead, we use the approach of Remark 1.8: we pass to a polynomial ring equipped with an appropriate Gauss norm and then return to $W(R)$ by taking a suitable quotient. The main difficulty in this approach is to transfer multiplicativity to the quotient norm; this requires construction of some good coset representatives.

Hypothesis 5.1. Throughout Section 5, equip $R$ with a power-multiplicative seminorm $\alpha$ bounded by the trivial norm, and write $\widehat{R}$ for the separated completion with respect to $\alpha$. (The restriction to the power-multiplicative case is made in light of Remark 4.8.) Choose $\pi=\sum_{i=0}^{\infty} p^{i}\left[\overline{\pi_{i}}\right]$ with $\alpha\left(\overline{\pi_{0}}\right) \leq$ $p^{-1}$ and $\overline{\pi_{1}}$ a unit in $R$; we write $\bar{\pi}$ as shorthand for $\bar{\pi}_{0}$. (In the language of [11], any such $\pi$ is primitive of degree 1.)

Definition 5.2. For $t \in[0,1]$, define the submultiplicative seminorm $H(\alpha, \pi, t)$ on $W(R)$ as the quotient norm on $W(R)[T] /(T-\pi) \cong W(R)$ induced by the $(t / p)$-Gauss extension of $\lambda(\alpha)$ to $W(R)[T]$. In case $\pi=p-[u]$ for $u \in R$ with $\alpha(u) \leq p^{-1}$, we denote $H(\alpha, \pi, t)$ also by $H(\alpha, u, t)$.

We will show shortly that $H(\alpha, \pi, t)$ is multiplicative whenever $\alpha$ is (Theorem $5.11(\mathrm{a}))$. For this, we will need some convenient coset representatives for the ideal $(T-\pi)$ in $W(R)[T]$.

Definition 5.3. We say that $x \in W(R)$ is stable (or $\alpha$-stable, in case we need to specify $\alpha$ ) if $x$ has the form $\sum_{i=0}^{\infty} p^{i}\left[\overline{x_{i}}\right]$ with either $\alpha\left(\overline{x_{i}}\right)=0$ for all $i \geq 0$, or $\alpha\left(\overline{x_{0}}\right)>p^{-i} \alpha\left(\overline{x_{i}}\right)$ for all $i>0$. For instance, any Teichmüller element is stable. 
REMARK 5.4. The term stable is chosen because of the following fact: for any stable $x \in W(R)$ with reduction $\bar{x}$ and any $\beta \in \mu^{-1}(\alpha)$, we have $\beta(x)=\lambda(\alpha)(x)=\alpha(\bar{x})$. Namely, this is immediate unless $\alpha(\bar{x})>0$, in which case

$$
\beta(x-[\bar{x}]) \leq \lambda(\alpha)(x-[\bar{x}])<\lambda(\alpha)([\bar{x}])=\alpha(\bar{x})=\beta([\bar{x}]) .
$$

Lemma 5.5. Assume that $R=\widehat{R}$. For any $x \in W(R)$, there exists $y=$ $\sum_{i=0}^{\infty} p^{i}\left[\overline{y_{i}}\right] \in W(R)$ with $x \equiv y(\bmod \pi)$ and $\alpha\left(\overline{y_{0}}\right) \geq \alpha\left(\overline{y_{i}}\right)$ for all $i>0$. In particular, $y$ is stable.

Proof. Note that $p^{-1}(\pi-[\bar{\pi}])$ is a unit in $W(R)$; let $w$ be its inverse. We construct $x_{0}, x_{1}, \ldots \in W(R)$ congruent to $x$ modulo $\pi$, as follows. Take $x_{0}=x$. Given $x_{i}$, write $x_{i}=\sum_{j=0}^{\infty} p^{j}\left[\overline{x_{i j}}\right]$ with $\overline{x_{i j}} \in R$, and put

$$
x_{i+1}=x_{i}-p^{-1} w\left(x_{i}-\left[\overline{x_{i 0}}\right]\right) \pi=\left[\overline{x_{i 0}}\right]-p^{-1} w\left(x_{i}-\left[\overline{x_{i 0}}\right]\right)[\bar{\pi}] .
$$

Let $N$ be the least nonnegative integer for which $\alpha\left(\overline{x_{N 0}}\right)>\alpha(\bar{\pi})^{N+1}$, or $\infty$ if no such integer exists. We check that $\Lambda(\alpha)\left(x_{i}\right) \leq \alpha(\bar{\pi})^{i}$ for $i \leq N$, by induction on $i$. The case $i=0$ is immediate. Given the claim for some $i \leq N$, we have

$$
\Lambda(\alpha)\left(x_{i+1}-\left[\overline{x_{i 0}}\right]\right) \leq \alpha(\bar{\pi}) \Lambda(\alpha)\left(x_{i}\right) \leq \alpha(\bar{\pi})^{i+1} .
$$

If $i<N$, this implies that $\Lambda(\alpha)\left(x_{i+1}\right) \leq \alpha(\bar{\pi})^{i+1}$, completing the induction. In addition, if $i=N<\infty$, then $\Lambda(\alpha)\left(x_{N+1}-\left[\overline{x_{N 0}}\right]\right)<\Lambda(\alpha)\left(\left[\overline{x_{N 0}}\right]\right)$, and so $x_{N+1}$ has the desired form. If $N=\infty$, then the series $\sum_{i=0}^{\infty} p^{-1} w\left(x_{i}-\left[\overline{x_{i 0}}\right]\right)$ converges $(p,[\bar{\pi}])$-adically to a limit $z$ satisfying $x=\pi z$, so we may take $y=0$.

Definition 5.6. Assume that $R=\widehat{R}$. Then $W(R)$ is $(p,[\bar{\pi}])$-adically complete, so any sum $\sum_{i=0}^{\infty} x_{i} \pi^{i}$ with $x_{i} \in W(R)$ converges to some limit $x$. We say that the sequence $x_{0}, x_{1}, \ldots$ forms a presentation of $x$ (with respect to $\pi$, or with respect to $u$ in case $\pi=p-[u])$. For $x \in W(R), H(\alpha, \pi, t)(x)$ may be computed as the infimum of

$$
\max _{i}\left\{(t / p)^{i} \lambda(\alpha)\left(x_{i}\right)\right\}
$$

over all presentations $x_{0}, x_{1}, \ldots$ of $x$.

A presentation $x_{0}, x_{1}, \ldots$ is stable (or $\alpha$-stable) if each $x_{i}$ is stable. Any $x \in W(R)$ admits a stable presentation (see Lemma 5.7 below). This will imply that the infimum defining $H(\alpha, \pi, t)(x)$ is always achieved (see Theorem 5.11(b) below). 
Lemma 5.7. If $R=\widehat{R}$, then every element of $W(R)$ admits a stable presentation.

Proof. Given $x, x_{0}, \ldots, x_{i-1} \in W(R)$, apply Lemma 5.5 to construct a stable $x_{i}$ congruent to $\left(x-\sum_{j=0}^{i-1} x_{j} \pi^{j}\right) / \pi^{i}$ modulo $\pi$. This process yields a stable presentation $x_{0}, x_{1}, \ldots$ of $x$.

Corollary 5.8. For each $x \in W(R)$ and each $\epsilon>0$, there exist a nonnegative integer $j$ and some stable elements $x_{0}, \ldots, x_{j} \in W(R)$ such that

$$
\lambda(\alpha)\left(x-\sum_{i=0}^{j} x_{i} \pi^{i}\right)<\epsilon .
$$

Proof. Apply Lemma 5.7 to construct a stable presentation $y_{0}, y_{1}, \ldots$ of $x$ in $W(\widehat{R})$. Choose $j$ with $p^{-j-1}<\epsilon$; then for each $i \in\{0, \ldots, j\}$, choose $x_{j} \in W(R)$ with $\lambda(\alpha)\left(y_{j}-x_{j}\right)<\epsilon$.

REMARK 5.9. It is unclear whether one can improve Lemma 5.7 to achieve a presentation using only Teichmüller elements rather than arbitrary stable elements. We suspect that this cannot be done, for reasons similar to those given in the erratum to [17].

Lemma 5.10. Assume that $R=\widehat{R}$. Let $x_{0}, x_{1}, \ldots, y_{0}, y_{1}, \ldots$ be presentations of some $x, y \in W(R)$ for which $x y \neq 0$. Then for all but finitely many $t \in[0,1]$, there exists a unique pair of indices $j, k$ maximizing $(t / p)^{j+k} \lambda(\alpha)\left(x_{j} y_{k}\right)$.

Proof. Since $x y \neq 0$, there must exist some indices $h, i$ for which $x_{h} y_{i} \neq 0$. Then for $t \in(0,1]$, the maximum of $(t / p)^{j+k} \lambda(\alpha)\left(x_{j} y_{k}\right)$ can only be achieved by pairs $(j, k)$ for which either $j+k \leq h+i$ or $p^{h+i-j-k} \geq \lambda(\alpha)\left(x_{h} y_{i}\right)$. This limits $(j, k)$ to a finite set independent of $t$; for any two pairs in that set, there is at most one value of $t$ for which both pairs of indices achieve the maximum. By excluding each such value, we obtain the desired result.

Theorem 5.11. Choose $t \in[0,1]$, and assume that $\alpha$ is powermultiplicative (resp., multiplicative).

(a) The function $H(\alpha, \pi, t)$ is a power-multiplicative (resp., multiplicative) seminorm on $W(R)$ bounded by $\lambda(\alpha)$.

(b) Assume that $R=\widehat{R}$. For any stable presentation $x_{0}, x_{1}, \ldots$ of $x \in W(R)$, $H(\alpha, \pi, t)(x)=\max _{i}\left\{(t / p)^{i} \lambda(\alpha)\left(x_{i}\right)\right\}$. 
(c) For $t \in[0, p \alpha(\bar{\pi})]$ with $p \alpha(\bar{\pi})>0$ and $c \in\left[1,1-\log _{p}(p \alpha(\bar{\pi}))\right]$, $H(\alpha, \pi, t)=H\left(\alpha^{1 / c}, \pi, p(t / p)^{1 / c}\right)^{c}$.

(d) For $t \in[p \alpha(\bar{\pi}), 1]$ with $t>0$, we have $H(\alpha, \pi, t)=\lambda\left(\alpha^{1 / c}\right)^{c}$ for $c=1-$ $\log _{p} t$. In particular, $H(\alpha, \pi, 1)=\lambda(\alpha)$. (For $t=0$, we obtain the same conclusion by interpreting $\lambda\left(\alpha^{1 / c}\right)^{c}$ for $c=+\infty$ as the restriction of $\alpha$ along $W(R) \rightarrow R$.)

Proof. We may assume throughout that $R=\widehat{R}$. Given $x, y \in W(R)$, apply Lemma 5.7 to construct stable presentations $x_{0}, x_{1}, \ldots, y_{0}, y_{1}, \ldots$ of $x, y$. We verify that

$$
H(\alpha, \pi, t)(x y) \geq \max _{j+k}\left\{(t / p)^{j+k} \lambda(\alpha)\left(x_{j} y_{k}\right)\right\} \quad(t \in[0,1]) .
$$

Suppose the contrary; then $x y \neq 0$. We must have a presentation $z_{0}, z_{1}, \ldots$ of $x y$ for which

$$
\max _{i}\left\{(t / p)^{i} \lambda(\alpha)\left(z_{i}\right)\right\}<\max _{j, k}\left\{(t / p)^{j+k} \lambda(\alpha)\left(x_{j} y_{k}\right)\right\}
$$

for some $t \in[0,1]$. Let $S$ be the set of $t \in(0,1]$ for which there are unique indices $j, k$ maximizing $(t / p)^{j+k} \lambda(\alpha)\left(x_{j} y_{k}\right)$. By Lemma 5.10 , the complement of $S$ in $[0,1]$ is finite. Since $(5.11 .2)$ holds for some $t$ and both sides of (5.11.2) are continuous in $t,(5.11 .2)$ must hold for some $t \in S$. Choose some such $t$, and put $s=(\log p) /(\log (p / t))$, so that $(t / p)^{i s}=p^{-i}$. We then have

$$
x_{j} y_{k} \pi^{j+k}=\sum_{i=0}^{\infty} z_{i} \pi^{i}-\sum_{\left(j^{\prime}, k^{\prime}\right) \neq(j, k)} x_{j^{\prime}} y_{k^{\prime}} \pi^{j^{\prime}+k^{\prime}},
$$

but

$$
\lambda\left(\alpha^{s}\right)\left(x_{j} y_{k} \pi^{j+k}\right)>\max _{i}\left\{\lambda\left(\alpha^{s}\right)\left(z_{i} \pi^{i}\right)\right\}, \quad \max _{\left(j^{\prime}, k^{\prime}\right) \neq(j, k)}\left\{\lambda\left(\alpha^{s}\right)\left(x_{j^{\prime}} y_{k^{\prime}} \pi^{j^{\prime}+k^{\prime}}\right)\right\} .
$$

This gives a contradiction, and (5.11.1) follows.

To deduce (a), note that from the definition, $H(\alpha, \pi, t)$ is evidently a submultiplicative seminorm bounded by $\lambda(\alpha)$. If $\alpha$ is multiplicative, then $H(\alpha, \pi, t)$ is multiplicative because (5.11.1) implies that $H(\alpha, \pi, t)(x y) \geq$ $H(\alpha, \pi, t)(x) H(\alpha, \pi, t)(y)$. Similarly, if $\alpha$ is power-multiplicative, then so is $H(\alpha, \pi, t)$. To deduce (b), apply (5.11.1) with $y=y_{0}=1$ and $y_{i}=0$ for $i>0$.

Suppose that $t \in[0, p \alpha(\bar{\pi})]$ with $p \alpha(\bar{\pi})>0$ and $c \in\left[1,1-\log _{p}(p \alpha(\bar{\pi}))\right]$. Since $c \leq 1-\log _{p}(p \alpha(\bar{\pi}))$, we have $\alpha^{1 / c}(\bar{\pi}) \leq p^{-1}$, so $H\left(\alpha^{1 / c}, \pi, p(t / p)^{1 / c}\right)$ 
is well defined. Since $c \geq 1$, any $\alpha$-stable element is also $\alpha^{1 / c}$-stable (as in Remark 5.4), so we may apply (b) to deduce (c).

To deduce $(\mathrm{d})$, note that $H(\alpha, \pi, 1) \leq \lambda(\alpha)$ from the definition of $H(\alpha, \pi, t)$ as a quotient norm, whereas $H(\alpha, \pi, 1) \geq \lambda(\alpha)$ from (b). Given this, if $t \in[p \alpha(\bar{\pi}), 1]$ with $t>0$ and $c=1-\log _{p} t$, then in particular $c \in[1,1-$ $\left.\log _{p}(p \alpha(\bar{\pi}))\right]$, so $(\mathrm{c})$ implies that $H(\alpha, \pi, t)=H\left(\alpha^{1 / c}, \pi, 1\right)^{c}=\lambda\left(\alpha^{1 / c}\right)^{c}$. This yields (d) for $t>0$; the case $t=0$ follows by continuity.

Corollary 5.12. For any $x \in W(R)$ with $\lambda(\alpha)(x) \neq 0$, the function $v_{x}(r)=-\log H\left(\alpha, \pi, e^{-r}\right)(x)$ on $[0,+\infty)$ is continuous, concave, nondecreasing, and piecewise affine with nonnegative integer slopes.

Proof. This is apparent from Theorem 5.11(b) and the existence of stable presentations in case $R=\widehat{R}$ (Lemma 5.7).

As an application of Corollary 5.12, we exhibit a computation which is not straightforward using stable presentations.

Lemma 5.13. For $u, u^{\prime} \in R$ with $\alpha(u), \alpha\left(u^{\prime}\right) \leq p^{-1}$ and $t \in[0,1]$,

$$
H(\alpha, u, t)\left(p-\left[u^{\prime}\right]\right)=\max \left\{t / p, H(\alpha, u, 0)\left(p-\left[u^{\prime}\right]\right)\right\}
$$

Proof. Consider the functions

$$
\begin{aligned}
& f(r)=-\log H\left(\alpha, u, e^{-r}\right)\left(p-\left[u^{\prime}\right]\right), \\
& g(r)=-\log \max \left\{e^{-r} / p, H(\alpha, u, 0)\left(p-\left[u^{\prime}\right]\right)\right\} .
\end{aligned}
$$

Note that $f$ and $g$ take the same value $\log p$ at $r=0$ and tend to the same (possibly infinite) limit as $r \rightarrow \infty$. In case $\alpha\left(u-u^{\prime}\right)=p^{-1},[u]-\left[u^{\prime}\right]$ is stable, so $[u]-\left[u^{\prime}\right], 1,0,0, \ldots$ is a stable presentation of $p-\left[u^{\prime}\right]$ with respect to $u$. By Theorem 5.11(b), $H(\alpha, u, t)\left(p-\left[u^{\prime}\right]\right)=p^{-1}$ for all $t \in[0,1]$, so $f=g$.

In case $\alpha\left(u-u^{\prime}\right)<p^{-1}$, we have $H(\alpha, u, 1)\left([u]-\left[u^{\prime}\right]\right)=\lambda(\alpha)\left([u]-\left[u^{\prime}\right]\right)<$ $p^{-1}$. Consequently, we have $H(\alpha, u, 1)\left(p-\left[u^{\prime}\right]\right)=t / p$ for $t$ close to 1 . This means that in a right neighborhood of $r=0, f(r)$ and $g(r)$ are both affine with slope 1. By Corollary 5.12, both functions are continuous, concave, nondecreasing, and piecewise affine with nonnegative integer slopes; hence, each function either persists with slope 1 forever or becomes constant after some point. Given this information plus the fact that $f$ and $g$ have the same limiting value, the two functions are forced to coincide. 
REMARK 5.14. Note that $H(\alpha, \pi, 0)$ is the quotient norm on $W(R) /(\pi)$ induced by $\lambda(\alpha)$. In particular, if $\alpha$ is a multiplicative norm, then $H(\alpha, \pi, 0)(x)=0$ if and only if $x$ is divisible by $\pi$.

Note also that any $\beta \in \mathcal{M}(W(R))$ with $\mu(\beta)=\alpha$ and $\beta(\pi)=0$ must equal $H(\alpha, \pi, 0)$. Namely, it suffices to check this assuming that $R=\widehat{R}$. Given $x \in W(R)$, apply Lemma 5.7 to construct a stable presentation $x_{0}, x_{1}, \ldots$ of $x$. By Theorem 5.11(b) and Remark 5.4, $H(\alpha, \pi, 0)(x)=\beta\left(x_{0}\right)=\beta(x)$.

REMARK 5.15. One consequence of Remark 5.14 is that if $\alpha$ is a multiplicative norm and $u, u^{\prime} \in R$ are such that $H(\alpha, u, 0)\left(p-\left[u^{\prime}\right]\right)=0$, then $p-\left[u^{\prime}\right]=y(p-[u])$ for some unit $y \in W(R)$. This implies that $H(\alpha, u, t)=$ $H\left(\alpha, u^{\prime}, t\right)$ for all $t \in[0,1]$; it does not imply that $u=u^{\prime}$ (see Example 6.11), but it does limit the possibilities for $u^{\prime}$, as in Remark 6.7 below.

We will need the following variant of Lemma 5.5.

LEMmA 5.16. For any $x \in W(R)$ and any $\epsilon>0$, there exists $y=$ $\sum_{i=0}^{\infty} p^{i}\left[\overline{y_{i}}\right] \in W(R)$ with $x \equiv y(\bmod \pi)$ and $\beta\left(\overline{y_{i}}\right) \leq \max \left\{\beta\left(\overline{y_{0}}\right), \epsilon\right\}$ for all $i>0$ and all $\beta \in \mathcal{M}(R)$.

Proof. Define $x=x_{0}, x_{1}, \ldots$ as in the proof of Lemma 5.5, and again write $x_{i}=\sum_{j=0}^{\infty} p^{j}\left[\overline{x_{i j}}\right]$. Take $n$ to be a nonnegative integer for which $\alpha(\bar{\pi})^{n} \leq \epsilon$, and put $y=x_{n}$. For $\beta \in \mathcal{M}(R)$, let $N$ be the least nonnegative integer for which $\beta\left(\overline{x_{N 0}}\right)>\beta(\bar{\pi})^{N+1}$, or $\infty$ if no such integer exists. By arguing as in the proof of Lemma 5.5, we see that if $n \leq N$, then $\Lambda(\beta)(y) \leq \beta(\bar{\pi})^{n} \leq \alpha(\bar{\pi})^{n} \leq \epsilon$; if instead $n>N$, then $\beta\left(\overline{x_{i 0}}\right)=\Lambda(\beta)\left(\overline{x_{i+1}}\right)=\beta\left(\overline{x_{N 0}}\right)$ for $i=N$, but also for $i>N$ by induction on $i$.

\section{§6. Newton polygons and factorizations}

The development of the basic algebra of polynomials over a complete nonarchimedean field is often phrased in the language of Newton polygons. One can develop a similar device to deal with the ring of Witt vectors over a perfect valuation ring; we use these to develop an analogue of the factorization of a polynomial over an algebraically closed field into linear constituents. This observation is due to Fargues and Fontaine [11] (see Remark 6.10).

Hypothesis 6.1. Throughout Section 6, let $\mathfrak{o}$ be the valuation ring of a perfect field of characteristic $p$ complete under a multiplicative norm $\alpha$. Equip $W(\mathfrak{o})$ with the norm $\lambda(\alpha)$, which is also multiplicative by Lemma 4.1. 
Definition 6.2. Let $W^{\dagger}($ Frac o $)$ denote the set of $x=\sum_{i=0}^{\infty} p^{i}\left[\overline{x_{i}}\right] \in$ $W($ Frac $\mathfrak{o})$ for which $p^{-i} \alpha\left(\overline{x_{i}}\right) \rightarrow 0$ as $i \rightarrow \infty$. For $T$ the set of nonzero Teichmüller lifts in $W(\mathfrak{o})$, we may identify $W^{\dagger}($ Frac $\mathfrak{o})$ with the completion of $T^{-1} W(\mathfrak{o})$ for the unique multiplicative extension of $\lambda(\alpha)$. We define stable elements of $W^{\dagger}($ Fraco $)$ using the same definition as in $W(\mathfrak{o})$ (see Definition 5.3).

For $x=\sum_{i=0}^{\infty} p^{i}\left[\overline{x_{i}}\right] \in W^{\dagger}($ Frac $\mathfrak{o})$ nonzero, the Newton polygon of $x$ is the lower boundary of the convex hull of the set

$$
\bigcup_{i=0}^{\infty}\left\{(a, b) \in \mathbb{R}^{2}: a \geq-\log \alpha\left(\overline{x_{i}}\right), b \geq i\right\},
$$

minus any segments of slope less than $(\log p)^{-1}$. The multiplicity of $r \in$ $\left[-(\log p)^{-1}, 0\right)$ in the Newton polygon of $x$ is the height of the segment of the Newton polygon of slope $r$, or 0 if no such segment exists.

Lemma 6.3. For $x, y \in W^{\dagger}($ Frac o $)$ nonzero and $r \in\left[-(\log p)^{-1}, 0\right)$, the multiplicity of $r$ in the Newton polygon of $x y$ is the sum of the multiplicities of $r$ in the Newton polygon of $x$ and $y$.

Proof. The argument is similar to the proofs that $\lambda(\alpha)$ and $H(\alpha, u, t)$ are multiplicative (Lemma 4.1 and Theorem $5.11(\mathrm{a})$ ), so we omit the details (see also [17, Lemma 2.1.7]).

COROLlary 6.4. The units in $W^{\dagger}($ Frac o $)$ are precisely the nonzero stable elements, which are in turn the elements with no slopes in their Newton polygons.

Proof. Any nonzero element $x$ of $W^{\dagger}($ Frac $\mathfrak{o})$ can be written uniquely as $[\bar{y}] z$ with $\bar{y} \in \mathfrak{o}$ nonzero and $z \in 1+p W^{\dagger}($ Frac $\mathfrak{o})$. If $x$ is stable, then $\lambda(\alpha)(z-1)<1$, so $z$ is a unit, as then is $x$. Conversely, if $x$ is a unit, then by Lemma 6.3 the multiplicity of each $r \in\left[-(\log p)^{-1}, 0\right)$ in the Newton polygon of $x$ is zero, so $x$ must be stable.

Lemma 6.5. For $u \in \mathfrak{o}$ with $\alpha(u) \leq p^{-1}$, the ideal $(p-[u])$ in $W(\mathfrak{o})$ is prime.

Proof. If $x y$ is divisible by $p-[u]$, then $H(\alpha, u, 0)(x y)=0$. Since $H(\alpha, u, 0)$ is multiplicative by Theorem $5.11(\mathrm{a})$, this forces either $H(\alpha, u, 0)(x)=0$ or $H(\alpha, u, 0)(y)=0$. Without loss of generality, suppose that $H(\alpha, u, 0)(x)=0 ;$ then by Remark 5.14, $x$ is divisible by $p-[u]$. 
Lemma 6.6. Suppose that $x \in W(\mathfrak{o})$ is nonzero and not stable.

(a) There exists an isometric embedding $\mathfrak{o} \rightarrow \mathfrak{o}_{0}$ of complete perfect valuation rings of characteristic $p$ such that in $W\left(\mathfrak{o}_{0}\right), x$ is divisible by $p-[u]$ for some $u \in \mathfrak{o}_{0}$ with $\alpha(u) \leq p^{-1}$.

(b) If Frac $\mathfrak{o}$ is algebraically closed, we may take $u \in \mathfrak{o}$.

Proof. In both cases, we may assume that $x$ is not divisible by $p$, because otherwise $u=0$ works. By rescaling $\alpha$, we may reduce to the case where $-(\log p)^{-1}$ has nonzero multiplicity in the Newton polygon of $x$. In this case, to prove (a), we will construct $u$ so that $\alpha_{0}(u)=p^{-1}$, for $\alpha_{0}$ the extended norm on $\mathfrak{o}_{0}$.

Let $S$ be the completion of $W^{\dagger}(\operatorname{Frac} \mathfrak{o})\left[p^{-1}\right]$ for the unique multiplicative extension of $\lambda(\alpha)$. Then $\kappa_{S}$ is a Laurent polynomial ring over $\kappa_{\mathfrak{o}}$ generated by the class of $x\left[\bar{x}^{-1}\right]-1$. It follows that $x$ is not a unit in $S$.

Equip $S /(x)$ with the quotient norm. Since $S /(x)$ is nonzero, $\mathcal{M}(S /(x)) \neq$ $\emptyset$ by Theorem 1.10. Choose $\beta \in \mathcal{M}(S /(x))$; it corresponds to an element of $\mu^{-1}(\alpha)$ with $\beta(p)=p^{-1}$ and $\beta(x)=0$. (Note that the condition on $\beta(p)$ would not have been guaranteed had we used $W^{\dagger}($ Frac o $)$ instead of $S$.) Restrict $\beta$ to $S$ and then to $W(\mathfrak{o})$; then use the isomorphism $W(\mathfrak{o})[T] /(T-$ $p) \cong W(\mathfrak{o})$ to further restrict $\beta$ to $W(\mathfrak{o})[T]$.

Since the restriction map $\psi^{*}: \mathcal{M}\left(W\left(\mathfrak{o}[\bar{T}]^{\text {perf }}\right)\right) \rightarrow \mathcal{M}(W(\mathfrak{o})[T])$ of Lemma 3.10 is surjective, we can extend $\beta$ to $\beta_{0} \in \mathcal{M}\left(W\left(\mathfrak{o}[\bar{T}]^{\text {perf }}\right)\right)$. Put $\alpha_{0}=\mu\left(\beta_{0}\right)$, let $\mathfrak{o}_{0}$ be the valuation ring of $\mathcal{H}\left(\alpha_{0}\right)$, and take $u$ to be the image of $[\bar{T}]$ in $\mathfrak{o}_{0}$. Since $\beta_{0}(x)=0, x$ is divisible by $p-[u]$ in $W\left(\mathfrak{o}_{0}\right)$ by Remark 5.14. This proves (a).

To prove (b), keep notation as above, but suppose by way of contradiction that $u \notin \mathfrak{o}$. Since Frac $\mathfrak{o}$ is algebraically closed, the restriction of the norm on $\mathfrak{o}_{0}$ to $\mathfrak{o}[\bar{T}]$ defines a point of $\mathcal{M}(\mathfrak{o}[\bar{T}])$ whose radius $r$ is positive. This in turn implies that if we equip $\mathfrak{o}_{0}[\bar{T}]^{\text {perf }}$ with the $r$-Gauss norm for the generator $\bar{T}-u$, then the map $\mathfrak{o}_{0}[\bar{T}]^{\text {perf }} \rightarrow \mathfrak{o}_{0} \widehat{\otimes} \mathfrak{o}_{0}$ taking $\mathfrak{o}$ to $\mathfrak{o} \otimes 1$ and $\bar{T}$ to $1 \otimes u$ is isometric.

Choose a nonnegative integer $n$ for which $p^{-p^{n}-1}<r$. For $i=0, \ldots, p^{n}$, let $\mathfrak{o}_{i}$ be a copy of $\mathfrak{o}_{0}$ in which $u_{i}$ denotes the element corresponding to $u$. Restrict $\alpha_{0}$ along the map $\mathfrak{o}_{0} \widehat{\bigotimes}_{\mathfrak{o}} \cdots \widehat{\bigotimes}_{\mathfrak{o}} \mathfrak{o}_{p^{n}} \rightarrow \mathfrak{o}_{0}$ to obtain a seminorm $\alpha^{\prime}$, and let $\mathfrak{o}^{\prime}$ be the valuation ring of $\mathcal{H}\left(\alpha^{\prime}\right)$.

For $0 \leq i<j \leq p^{n}$, we have $H\left(\alpha^{\prime}, u_{0}, 0\right)\left(p-\left[u_{i}\right]\right)=H\left(\alpha^{\prime}, u_{0}, 0\right)\left(p-\left[u_{j}\right]\right)=$ 0 , so $\left[\left(u_{j} / u_{i}\right)^{p^{-n}}\right]$ maps to a $p^{n}$ th root of unity in $\mathcal{H}\left(H\left(\alpha^{\prime}, u_{0}, 0\right)\right)$. If this root were 1 , then by Remark $5.14,\left[\left(u_{j} / u_{i}\right)^{p^{-n}}\right]-1$ would be divisible by $p-\left[u_{0}\right]$ 
in $W\left(\mathfrak{o}^{\prime}\right)$, which would imply that $\alpha^{\prime}\left(\left(u_{j} / u_{i}\right)^{p^{-n}}-1\right) \leq p^{-1}$ and $\alpha^{\prime}\left(u_{j}-u_{i}\right) \leq$ $p^{-p^{n}} \alpha(u)<r$; however, this would contradict the description of $\alpha$ from the preceding paragraph. It follows that $\left[\left(u_{j} / u_{i}\right)^{p^{-n}}\right]$ maps to a nontrivial $p^{n}$ th root of unity in $\mathcal{H}\left(H\left(\alpha^{\prime}, u_{0}, 0\right)\right)$, but by the pigeonhole principle, this cannot hold simultaneously for all $i, j$. The resulting contradiction forces $u \in \mathfrak{o}$; this yields (b).

REMARK 6.7. By a similar argument to the proof of Lemma 6.6(b), one may show the following. For $u \in \mathfrak{o}$ with $\alpha(u) \leq p^{-1}$, for each $\epsilon>0$, the set of $u^{\prime} \in \mathfrak{o}$ with $H(\alpha, u, 0)\left(p-\left[u^{\prime}\right]\right)=0$ is contained in finitely many residue classes modulo elements of norm at most $\epsilon$. More precisely, if $p^{-p^{n}-1}<\epsilon$, there are at most $p^{n}$ such classes.

Theorem 6.8. Assume that Frac o is algebraically closed. For $x \in W(\mathfrak{o})$ nonzero and not stable, we can write $x=y\left(p-\left[u_{1}\right]\right) \cdots\left(p-\left[u_{n}\right]\right)$ for some nonzero stable $y \in W(\mathfrak{o})$ and some $u_{1}, \ldots, u_{n} \in \mathfrak{o}$ with $\alpha\left(u_{1}\right), \ldots, \alpha\left(u_{n}\right)<$ $p^{-1}$.

Proof. We may divide out powers of $p$ as needed to reduce to the case where $x$ is not divisible by $p$. Let $n$ be the sum of all multiplicities in the Newton polygon of $x$; this is a nonnegative integer. We check that for $m=0, \ldots, n$, we can find $u_{1}, \ldots, u_{m} \in \mathfrak{o}$ such that $x$ is divisible by $(p-$ $\left.\left[u_{1}\right]\right) \cdots\left(p-\left[u_{m}\right]\right)$. This proceeds by induction on $m$, with empty base case $m=0$. For the induction step, since $m<n$, by Lemma 6.3 , the sum of all multiplicities in the Newton polygon of $x_{m}=x /\left(\left(p-\left[u_{1}\right]\right) \cdots\left(p-\left[u_{m}\right]\right)\right)$ is nonzero, so $x_{m}$ cannot be stable. We may thus apply Lemma 6.6 to construct $u_{m+1}$ of the desired form.

Given $u_{1}, \ldots, u_{n}$ as above, put $y=x /\left(\left(p-\left[u_{1}\right]\right) \cdots\left(p-\left[u_{n}\right]\right)\right) \in W\left(\mathfrak{o}_{1}\right)$. By Lemma 6.3, the Newton polygon of $y$ has no slopes, so $y$ is stable. This gives the desired factorization.

REMARK 6.9. For a fixed choice of $u \in \mathfrak{o}$ with $\alpha(u) \leq p^{-1}$, one can also define Newton polygons which keep track of the seminorms $H(\alpha, u, t)$, either by examining stable presentations or by taking the concave duals of the graphs of the functions $v_{r}(x)$ from Corollary 5.12. We leave it to the reader to formulate and verify the multiplicativity property in this case.

By analogy with the theory of Newton polygons for polynomials over a complete nonarchimedean field, one may expect that for $x \in W(\mathfrak{o})$ nonzero, we can use the Newton polygon to read off some information about the factors occurring in the representation $x=y\left(p-\left[u_{1}\right]\right) \cdots\left(p-\left[u_{n}\right]\right)$ given by 
Theorem 6.8. Again, this is equivalent to a statement about the function $v_{r}(x)$, which may be deduced from Lemma 5.13: the right slope of $v_{r}(x)$ at $r$ counts the number of indices $i$ for which $H(\alpha, u, 0)\left(p-\left[u_{i}\right]\right)<e^{-r} / p$.

REMARK 6.10. A similar analysis of elements of $W(\mathfrak{o})$, including a somewhat more constructive proof of Theorem 6.8, appears in the development of $p$-adic Hodge theory given by Fargues and Fontaine [11].

As an application of Theorem 6.8, we can produce an example of distinct $u, u^{\prime} \in \mathfrak{o}$ with $\alpha(u)=\alpha\left(u^{\prime}\right)=p^{-1}$ for which $p-[u]$ and $p-\left[u^{\prime}\right]$ generate the same ideal in $W(\mathfrak{o})$, as promised in Remark 5.15. This example is crucial in $p$-adic Hodge theory, as in [20] or [11].

EXAmple 6.11. Suppose that there exists $\bar{x} \in \mathfrak{o}$ with $\alpha(\bar{x})=p^{-p /(p-1)}$. Put

$$
\pi=\sum_{j=0}^{p-1}[1+\bar{x}]^{j / p} \in W(\mathfrak{o}),
$$

and write $\pi=\sum_{i=0}^{\infty} p^{i}\left[\overline{\pi_{i}}\right]$. Then $\alpha\left(\overline{\pi_{0}}\right)=p^{-1}$ and $\alpha\left(\overline{\pi_{1}}\right)=1$, so by Theorem 6.8 we can write $\pi=(p-[u]) y$ for some $u \in \mathfrak{o}$ with $\alpha(u)=p^{-1}$ and some unit $y \in W(\mathfrak{o})$. Note that $\pi\left(1-[1+\bar{x}]^{1 / p}\right)=1-[1+\bar{x}]$, so 1 and $[1+\bar{x}]$ have the same image in $W(\mathfrak{o}) /(p-[u])=W(\mathfrak{o}) /(\pi)$. Consequently, $H(\alpha, u, 0)\left(p-\left[u^{\prime}\right]\right)=0$ for $u^{\prime}=u(1+\bar{x})^{\gamma}$ for any $\gamma \in \mathbb{Z}$, and by continuity also for any $\gamma \in \mathbb{Z}_{p}$ if we use the binomial series to define $(1+\bar{x})^{\gamma}$. By Remark 5.14, $p-[u]$ and $p-\left[u^{\prime}\right]$ generate the same ideal in $W(\mathfrak{o})$.

\section{§7. Restriction of Gauss norms}

We are now ready to construct a strong deformation retract between the spectra of $R$ and $W(R)$. We cannot directly imitate the construction for polynomial rings, for lack of an analogue of (2.3.1) (see Remark 3.11). We thus instead follow the approach suggested in Remark 2.8. Given an element of $\mathcal{M}(W(R))$, we express it as the restriction from a larger Witt ring of a seminorm of the form $H(\alpha, u, 0)$ and then define the homotopy by restricting the corresponding seminorms $H(\alpha, u, t)$. Before embarking on this construction, we read off a key continuity property from the construction of the seminorms $H(\alpha, u, t)$.

THEOREM 7.1. Equip $R$ with a power-multiplicative norm $\alpha$ bounded above by the trivial norm, equip $W(R)$ with the power-multiplicative norm 
$\lambda(\alpha)$, and choose $\pi=\sum_{i=0}^{\infty} p^{i}\left[\overline{\pi_{i}}\right]$ with $\alpha\left(\overline{\pi_{0}}\right) \leq p^{-1}$ and $\overline{\pi_{1}}$ a unit in $R$. Then the map

$$
H(\cdot, \pi, \cdot): \mathcal{M}(R) \times[0,1] \rightarrow \mathcal{M}(W(R))
$$

defined by Theorem 5.11(a) is continuous.

Proof. To check continuity, we must check that for each $x \in W(R)$ and each $\epsilon>0$, the sets

$$
\begin{aligned}
& \{(\gamma, t) \in \mathcal{M}(R) \times[0,1]: H(\gamma, \pi, t)(x)>\epsilon\} \\
& \{(\gamma, t) \in \mathcal{M}(R) \times[0,1]: H(\gamma, \pi, t)(x)<\epsilon\}
\end{aligned}
$$

are open. Pick $\left(\gamma_{0}, t_{0}\right)$ in one of these sets. By Corollary 5.8, we can find $\gamma_{0}$-stable elements $x_{0}, \ldots, x_{j} \in W(R)$ such that

$$
\lambda\left(\gamma_{0}\right)\left(x-\sum_{i=0}^{j} x_{i} \pi^{i}\right)<\epsilon / 2 .
$$

We may further ensure that each nonzero $x_{i}$ satisfies $\lambda\left(\gamma_{0}\right)\left(x_{i}\right)>0$.

Given some nonzero $x_{i}$, write $x_{i}=\sum_{k=0}^{\infty} p^{k}\left[\overline{x_{i k}}\right]$. Choose an integer $h$ for which $\gamma_{0}\left(\overline{x_{i 0}}\right)>p^{-h}$. Then the set of $\gamma \in \mathcal{M}(W(R))$ for which $\gamma\left(\overline{x_{i 0}}\right)>$ $p^{-h}$ and $p^{-k} \gamma\left(\overline{x_{i k}}\right)<\gamma\left(\overline{x_{i 0}}\right)$ for $k=1, \ldots, h-1$ is open and contains $\gamma_{0}$. Consequently, there is an open neighborhood $U$ of $\gamma_{0}$ in $\mathcal{M}(W(R))$ such that $x_{0}, \ldots, x_{j}$ are $\gamma$-stable for each $\gamma \in U$.

For $(\gamma, t) \in U \times[0,1]$, applying Theorem 5.11(b) over the ring $\mathfrak{o}_{\mathcal{H}(\gamma)}$ yields

$$
\max \{\epsilon / 2, H(\gamma, \pi, t)(x)\}=\max \left\{\epsilon / 2, \max _{i}\left\{(t / p)^{i} \gamma\left(\overline{x_{i 0}}\right)\right\}\right\} .
$$

There thus exist an open neighborhood $V$ of $\gamma_{0}$ and an open interval $I$ containing $t_{0}$ for which for each pair $(\gamma, t) \in V \times I, H(\gamma, \pi, t)(x)$ and $H\left(\gamma_{0}, \pi, t_{0}\right)(x)$ are either both greater than $\epsilon$ or both less than $\epsilon$. This yields the desired result.

Corollary 7.2. With notation as in Theorem 7.1, the map $H(\cdot, \pi, 0)$ induces a homeomorphism $\mathcal{M}(R) \rightarrow \mathcal{M}(W(R) /(\pi))$, whose inverse is induced by $\mu$. Moreover, any subset of $\mathcal{M}(R)$ is Weierstrass (resp., Laurent, rational) if and only if its image in $\mathcal{M}(W(R) /(\pi))$ is Weierstrass (resp., Laurent, rational). 
Proof. The first statement is immediate from Theorems 4.5 and 7.1 and Remark 5.14. For the second statement, observe that from the proof of Theorem 4.5(a), the image of a Weierstrass (resp., Laurent, rational) subspace of $\mathcal{M}(R)$ is again such a subspace. We establish the converse only for a rational subspace, because the other cases behave similarly; we may also assume that $R$ is complete under $\alpha$. Let

$$
U=\left\{\gamma \in \mathcal{M}(W(R) /(\pi)): \gamma\left(f_{i}\right) \leq p_{i} \gamma(g)(i=1, \ldots, n)\right\}
$$

be the rational subspace defined by some $f_{i}, g \in W(R)$ generating the unit ideal in $W(R) /(\pi)$ and some $p_{i}>0$. Apply Remark 1.15 to find $\epsilon>0$ for which $\gamma(g)>\epsilon$ for all $\gamma \in U$. By Lemma 5.16, we can find ${\overline{f_{1}}}^{\prime}, \ldots,{\overline{f_{n}}}^{\prime}, \bar{g}^{\prime} \in R$ such that for all $\gamma \in \mathcal{M}(W(R) /(\pi))$,

$$
\gamma\left(f_{i}-\left[{\overline{f_{i}}}^{\prime}\right]\right) \leq p^{-1} \max \left\{\gamma\left(f_{i}\right), p_{i} \epsilon\right\}, \quad \gamma\left(g-\left[\bar{g}^{\prime}\right]\right) \leq p^{-1} \max \{\gamma(g), \epsilon\} .
$$

(More precisely, apply Lemma 5.16 with $x=f_{1}, \ldots, f_{n}, g$, and let $f_{1}^{\prime}, \ldots$, $f_{n}^{\prime}, g^{\prime}$ be the resulting values of $y$; then reduce modulo $p$.) By Remark 1.15, $\left[{\overline{f_{1}}}^{\prime}\right], \ldots,\left[\overline{f_{n}}\right],\left[\bar{g}^{\prime}\right]$ also generate the unit ideal in $W(R) /(\pi)$, so ${\overline{f_{1}}}^{\prime}, \ldots,{\overline{f_{n}}}^{\prime}$, $\bar{g}^{\prime}, \bar{\pi}$ generate the unit ideal in $R$; the same is then true without $\bar{\pi}$.

For $\gamma \in \mathcal{M}(W(R) /(\pi))$ corresponding to $\beta \in \mathcal{M}(R), \gamma(g) \geq \epsilon$ if and only if $\beta\left(\bar{g}^{\prime}\right) \geq \epsilon$, in which case $\gamma(g)=\beta\left(\bar{g}^{\prime}\right)$. Also, in this case, $\gamma\left(f_{i}\right) \leq p_{i} \gamma(g)$ if and only if $\beta\left({\overline{f_{i}}}^{\prime}\right) \leq p_{i} \gamma(g)=p_{i} \beta\left(\bar{g}^{\prime}\right)$. Consequently, $U$ corresponds to the rational subspace

$$
\left\{\beta \in \mathcal{M}(R): \beta\left({\overline{f_{i}}}^{\prime}\right) \leq p_{i} \beta\left(\bar{g}^{\prime}\right)(i=1, \ldots, n)\right\},
$$

as desired.

REMARK 7.3. Corollary 7.2 defines a remarkable section of the projection $\mu$ : it is a homeomorphism of topological spaces, but one of the underlying rings is of characteristic $p$, while the other is not. We plan to explore the relationship between these rings in subsequent work.

To use Theorem 7.1 to define the desired homotopy, we argue as in Remark 2.8. However, we must overcome a technical complication that does not occur there, because the analogous construction here is not a priori well defined.

Lemma 7.4. Define $\psi: W(R)[T] \rightarrow W\left(R[\bar{T}]^{\text {perf }}\right)$ as in Lemma 3.10. Choose $\beta_{1}, \beta_{2} \in \mathcal{M}\left(W\left(R[\bar{T}]^{\text {perf }}\right)\right)$ with $\beta_{1}(p-[\bar{T}])=\beta_{2}(p-[\bar{T}])=0$ and $\psi^{*}\left(\beta_{1}\right)=\psi^{*}\left(\beta_{2}\right)$. Then for all $t \in[0,1]$, the restrictions of $H\left(\mu\left(\beta_{1}\right),[\bar{T}], t\right)$ and $H\left(\mu\left(\beta_{2}\right),[\bar{T}], t\right)$ to $W(R)$ coincide. 
Proof. By Lemma 1.20, for $S=W\left(R[\bar{T}]^{\text {perf }}\right) \bigotimes_{W(R)} W\left(R[\bar{T}]^{\text {perf }}\right)$, there exists $\beta_{3} \in \mathcal{M}(S)$ restricting to $\beta_{1}, \beta_{2}$ on the tensorands. (For $\beta=\psi^{*}\left(\beta_{1}\right)$, one can also argue directly that $\mathcal{H}\left(\beta_{1}\right) \widehat{\otimes}_{\mathcal{H}(\beta)} \mathcal{H}\left(\beta_{2}\right) \neq 0$ using the fact that $\mathcal{H}\left(\beta_{i}\right)$ is the completion of an algebraic extension of $\mathcal{H}(\beta)$.) We may identify $S$ with a dense subring of $W\left(R\left[\overline{T_{1}}, \overline{T_{2}}\right]^{\text {perf }}\right)$ by identifying $[\bar{T}] \otimes 1$ with $\left[\overline{T_{1}}\right]$ and $1 \otimes[\bar{T}]$ with $\left[\overline{T_{2}}\right]$; we may then extend $\beta_{3}$ to $W\left(R\left[\overline{T_{1}}, \overline{T_{2}}\right]^{\text {perf }}\right)$ by continuity.

For $i=1,2,3$, put $\alpha_{i}=\mu\left(\beta_{i}\right)$, let $\mathfrak{o}_{i}$ be the valuation ring of $\mathcal{H}\left(\alpha_{i}\right)$, and extend $\beta_{i}$ to a multiplicative seminorm on $W\left(\mathfrak{o}_{i}\right)$. Then $\beta_{3}\left(p-\left[\overline{T_{1}}\right]\right)=\beta_{3}(p-$ $\left.\left[\overline{T_{2}}\right]\right)=0$, so by Remark 5.15, we have $H\left(\alpha_{3}, \overline{T_{1}}, t\right)=H\left(\alpha_{3}, \overline{T_{2}}, t\right)$ for all $t \in[0,1]$. Since $H\left(\alpha_{3}, \overline{T_{i}}, t\right)$ restricts to $H\left(\alpha_{i}, \overline{T_{i}}, t\right)$, this proves the claim.

Definition 7.5. Define $\psi: W(R)[T] \rightarrow W\left(R[\bar{T}]^{\text {perf }}\right)$ as in Lemma 3.10. Given $\beta \in \mathcal{M}(W(R))$, restrict $\beta$ along $W(R)[T] \rightarrow W(R)[T] /(p-T) \cong$ $W(R)$, and then apply Lemma $3.10(\mathrm{~b})$ to extend $\beta$ to $\beta_{1} \in \mathcal{M}\left(W\left(R[\bar{T}]^{\text {perf }}\right)\right)$. By Lemma 7.4, for $t \in[0,1]$, the restriction of $H\left(\mu\left(\beta_{1}\right), \bar{T}, t\right)$ to $W(R)$ is independent of the choice of $\beta_{1}$; we call this restriction $H(\beta, t)$. It is a multiplicative seminorm by Theorem 5.11(a); its formation is evidently compatible with restriction along bounded homomorphisms.

REMARK 7.6. For $\beta \in \mathcal{M}(W(R))$, let $\tilde{\beta}$ be the spectral seminorm associated to the product seminorm on $W(R)[T] /(T-p) \bigotimes_{W(R)[T]} W\left(R[\bar{T}]^{\text {perf }}\right)$ using $\beta$ on the first factor; this equals the supremum over all extensions of $\beta$ to $W\left(R[\bar{T}]^{\text {perf }}\right.$ ) (see Definition 1.11). Consequently, by Lemma 7.4 , we may compute $H(\beta, t)$ by restricting the spectral seminorm associated to the quotient norm on

$$
W\left(R[\bar{T}]^{\text {perf }}\right)[U] /(U-p+[\bar{T}])
$$

induced by the $(t / p)$-Gauss extension of $\lambda(\mu(\tilde{\beta}))$.

REMARK 7.7. One consequence of Remark 7.6 is monotonicity: for $\beta, \beta^{\prime} \in$ $\mathcal{M}(W(R))$ and $t, t^{\prime} \in[0,1]$ with $\beta \geq \beta^{\prime}$ and $t \geq t^{\prime}$, we have $H(\beta, t) \geq H\left(\beta^{\prime}, t^{\prime}\right)$. This is not evident from Definition 7.5 because Lemma 3.10 does not guarantee that $\beta, \beta^{\prime}$ admit extensions $\beta_{1}, \beta_{1}^{\prime}$ to $W\left(R[\bar{T}]^{\text {perf }}\right)$ which satisfy $\beta_{1} \geq \beta_{1}^{\prime}$.

We obtain the following analogue of Theorem 2.5.

TheOREM 7.8. The map $H: \mathcal{M}(W(R)) \times[0,1] \rightarrow \mathcal{M}(W(R))$ given in Definition 7.5 is continuous and has the following additional properties.

(a) For $\beta \in \mathcal{M}(W(R)), H(\beta, 0)=\beta$.

(b) For $\beta \in \mathcal{M}(W(R)), H(\beta, 1)=(\lambda \circ \mu)(\beta)$. 
(c) For $\beta \in \mathcal{M}(W(R))$ and $t \in[0,1], \mu(H(\beta, t))=\mu(\beta)$.

(d) For $\beta \in \mathcal{M}(W(R))$ and $s, t \in[0,1], H(H(\beta, s), t)=H(\beta, \max \{s, t\})$.

Proof. Let $\alpha$ be the $\bar{T}$-adic norm on $R[\bar{T}]^{\text {perf }}$ for the normalization $\alpha(\bar{T})=$ $p^{-1}$. Equip $W\left(R[\bar{T}]^{\text {perf }}\right) /(p-[\bar{T}])$ with the quotient norm induced by $\lambda(\alpha)$. We then obtain a continuous map

$$
\mathcal{M}\left(W\left(R[\bar{T}]^{\text {perf }}\right) /(p-[\bar{T}])\right) \times[0,1] \rightarrow \mathcal{M}(W(R))
$$

by applying first $\mu \times$ id (which is continuous by Theorem 4.5 ) and then $H(\cdot, \bar{T}, \cdot)$ (which is continuous by Theorem 7.1 ) and then restricting along the inclusion $W(R) \rightarrow W\left(R[\bar{T}]^{\text {perf }}\right)$.

By Lemma 7.4, we have a commutative diagram

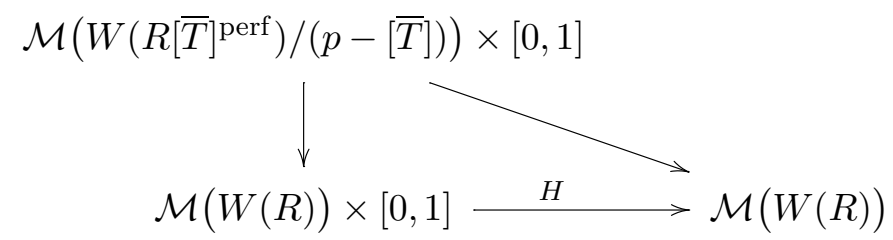

in which the diagonal arrow is continuous and the vertical arrow is a quotient map by Lemma $3.10(\mathrm{~b})$. This yields the continuity of $H$. We deduce (a) from Remark 5.14, (b) from Theorem 5.11(d), and (c) from Remark 5.4 (or, more precisely, by noting that $\mu(H(\beta, t))(\bar{x})=H(\beta, t)([\bar{x}])$ and that because Teichmüller elements are stable, the latter equals $\beta([\bar{x}])=\mu(\beta)(\bar{x}))$.

To establish (d), we may follow the construction of Definition 7.5 to reduce to the case where $R=\mathfrak{o}$ is the valuation ring of a perfect field complete for a multiplicative norm $\gamma$, and $\beta(p-[u])=0$ for some $u \in \mathfrak{o}$ with $\gamma(u) \leq p^{-1}$. By Remark 5.14 again, this ensures that $\beta=H(\gamma, u, 0)$. This formula defines an extension of $\beta$ to $W\left(\mathfrak{o}_{1}\right)$ whenever $\mathfrak{o}_{1}$ is the valuation ring of a complete field extension of Frac $\mathfrak{o}$; we may thus reduce to the case where Frac $\mathfrak{o}$ is algebraically closed.

In this case, by Theorem 6.8, any nonzero element of $W(\mathfrak{o})$ factors as a stable element times a product of finitely many terms each of the form $p-\left[u^{\prime}\right]$ for some $u^{\prime} \in \mathfrak{o}$ with $\gamma\left(u^{\prime}\right) \leq p^{-1}$. To establish (d), we thus need only check that the functions

$$
\begin{aligned}
& f(r)=-\log H\left(H(\beta, s), e^{-r}\right)\left(p-\left[u^{\prime}\right]\right), \\
& g(r)=-\log H\left(\beta, \max \left\{s, e^{-r}\right\}\right)\left(p-\left[u^{\prime}\right]\right)
\end{aligned}
$$


are identically equal. By Lemma 5.13, $f$ and $g$ are both continuous, concave, nondecreasing, and piecewise linear with slopes in $\{0,1\}$. Moreover, they take the same value at $r=0$ (namely, $\log p$ ) and have the same limiting value as $r \rightarrow \infty$ (because $H(H(\beta, s), 0)=H(\beta, s)$ by (a)). Consequently, they must coincide.

Corollary 7.9. Each subset of $\mathcal{M}(R)$ has the same homotopy type as its inverse image in $\mathcal{M}(W(R))$ under $\mu$.

We have the following analogue of Lemma 2.4.

Lemma 7.10. For $\alpha \in \mathcal{M}(R)$ and $s, t \in[0,1], H(H(\alpha, u, s), t)=H(\alpha, u$, $\max \{s, t\})$.

Proof. Put $\beta=H(\alpha, u, 0)$, and set notation as in Definition 7.5. Then $\beta_{1}(p-[u])=\beta_{1}(p-[\bar{T}])=0$, so $H(\alpha, u, s)=H(\beta, s)$ by Remark 5.15. By Theorem 7.8(d),

$$
H(H(\alpha, u, s), t)=H(H(\beta, s), t)=H(\beta, \max \{s, t\})=H(\alpha, u, \max \{s, t\}),
$$

as desired.

We also have the following analogue of Theorem 2.11. Again, this depends on an analysis of the fibers of $\mu$, which we carry out in Section 8.

Definition 7.11. For $\beta \in \mathcal{M}(W(R))$, the set of $s \in[0,1]$ for which $H(\beta, s)=\beta$ is nonempty (because it contains 0 ) and closed (by continuity), so it has a greatest element. As in Definition 2.10, we call this greatest element the radius of $\beta$, and denote it by $r(\beta)$.

Theorem 7.12. Suppose that $\beta, \gamma \in \mathcal{M}(W(R))$ are such that $\beta \geq \gamma$ and $\mu(\beta)=\mu(\gamma)$. Then $\beta=H(\gamma, r(\beta))$.

Proof. Put $\alpha=\mu(\beta)=\mu(\gamma)$, let $\mathfrak{o}$ be the valuation ring of $\mathcal{H}(\alpha)$, and identify $\beta, \gamma$ with the corresponding points in $\mu^{-1}(\alpha) \subseteq \mathcal{M}(W(\mathfrak{o}))$. These identifications are compatible with the formation of $H(\cdot, t)$; in particular, they do not change the radius of $\beta$. It thus suffices to check the case $R=\mathfrak{o}$ (see Lemma 8.12 below).

Corollary 7.13. For $\beta, \gamma \in \mathcal{M}(W(R))$ satisfying $\mu(\beta)=\mu(\gamma)$ and $\beta \geq$ $\gamma$, we have $r(\beta) \geq r(\gamma)$, with equality if and only if $\beta=\gamma$.

Proof. For $t \in[0, r(\gamma)]$, by Theorems $7.8(\mathrm{~d})$ and 7.12, we have

$$
H(\beta, t)=H(H(\gamma, r(\beta)), t)=H(H(\gamma, t), r(\beta))=H(\gamma, r(\beta))=\beta,
$$

so $r(\beta) \geq r(\gamma)$. If equality holds, then $\gamma=H(\gamma, r(\gamma))=H(\gamma, r(\beta))=\beta$. 


\section{$\S 8$. Structure of fibers}

We conclude with a description of the fibers of the map $\mu: \mathcal{M}(W(R)) \rightarrow$ $\mathcal{M}(R)$ similar to the description of $\mathcal{M}(K[T])$ given in Section 2. This will allow us to establish Theorem 7.12, thus giving a combinatorial interpretation of the fibers of $\mu$.

Hypothesis 8.1. Throughout Section 8, retain Hypothesis 6.1. In addition, let $\tilde{\mathfrak{o}}$ be the valuation ring of the completion of an algebraic closure of Frac $\mathfrak{o}$, equipped with the unique multiplicative extension $\tilde{\alpha}$ of $\alpha$, and equip $W(\tilde{\mathfrak{o}})$ with the multiplicative norm $\lambda(\tilde{\alpha})$.

Definition 8.2. For $u \in \tilde{\mathfrak{o}}$ with $\tilde{\alpha}(u) \leq p^{-1}$ and $t \in[0,1]$, let $\tilde{\beta}_{u, t} \in \mu^{-1}(\tilde{\alpha})$ be the seminorm $H(\tilde{\alpha}, u, t)$ of Theorem 5.11. Let $\beta_{u, t}$ be the restriction of $\tilde{\beta}_{u, t}$ to $W(\mathfrak{o})$.

Before studying the $\beta_{u, t}$, we must work out some facts about the $\tilde{\beta}_{u, t}$ which are not quite as obvious as their counterparts for $K[T]$.

Lemma 8.3. For $u, u^{\prime} \in \tilde{\mathfrak{o}}$ with $\tilde{\alpha}(u), \tilde{\alpha}\left(u^{\prime}\right) \leq p^{-1}$ and $t \in(0,1]$, the following conditions are equivalent.

(a) We have $\tilde{\beta}_{u, t}=\tilde{\beta}_{u^{\prime}, t}$.

(b) We have $\tilde{\beta}_{u, t} \geq \tilde{\beta}_{u^{\prime}, t}$.

(c) We have $\tilde{\beta}_{u, t} \geq \tilde{\beta}_{u^{\prime}, 0}$.

(d) We have $t / p \geq \tilde{\beta}_{u^{\prime}, 0}(p-[u])$.

Proof. Clearly (a) $\Longrightarrow(\mathrm{b}) \Longrightarrow(\mathrm{c}) \Longrightarrow(\mathrm{d})$; it remains to check that (d) $\Longrightarrow$ (a). If $t \geq \max \left\{p \tilde{\alpha}(u), p \tilde{\alpha}\left(u^{\prime}\right)\right\}$, then $\tilde{\beta}_{u, t}=\tilde{\beta}_{u^{\prime}, t}$ by Theorem $5.11(\mathrm{~d})$, so (a) always holds. We may thus assume that $t<\max \left\{p \tilde{\alpha}(u), p \tilde{\alpha}\left(u^{\prime}\right)\right\}$ hereafter.

By $(\mathrm{d})$, we have $\tilde{\beta}_{u^{\prime}, 0}\left([u]-\left[u^{\prime}\right]\right) \leq t / p$. That is, there exists $y \in W(\tilde{\mathfrak{o}})$ for which

$$
\lambda(\tilde{\alpha})\left([u]-\left[u^{\prime}\right]+y\left(p-\left[u^{\prime}\right]\right)\right) \leq t / p .
$$

Note that we cannot have $\tilde{\alpha}(u) \neq \tilde{\alpha}\left(u^{\prime}\right)$, because then $[u]-\left[u^{\prime}\right]$ would be stable and we would derive the contradiction $\max \left\{\tilde{\alpha}(u), \tilde{\alpha}\left(u^{\prime}\right)\right\}=\lambda(\tilde{\alpha})([u]-$ $\left.\left[u^{\prime}\right]\right)=\tilde{\beta}_{u^{\prime}, 0}\left([u]-\left[u^{\prime}\right]\right) \leq t / p$. We must thus have $\tilde{\alpha}(u)=\tilde{\alpha}\left(u^{\prime}\right)$. For $\bar{y}$ the reduction of $y$ modulo $p$, we cannot have $\tilde{\alpha}(1+\bar{y})<1$, or else we would derive the contradiction $\max \left\{\tilde{\alpha}(u), \tilde{\alpha}\left(u^{\prime}\right)\right\}=\tilde{\alpha}\left(u-(1+\bar{y}) u^{\prime}\right) \leq t / p$. We deduce that $1+y$ is a unit in $W(\tilde{\mathfrak{o}})$.

Put $y^{\prime}=y /(1+y)$; then

$$
[u]-\left[u^{\prime}\right]+y^{\prime}(p-[u])=(1+y)^{-1}\left([u]-\left[u^{\prime}\right]+y\left(p-\left[u^{\prime}\right]\right)\right),
$$


so $\lambda(\tilde{\alpha})\left([u]-\left[u^{\prime}\right]+y^{\prime}(p-[u])\right) \leq t / p$, and hence $\tilde{\beta}_{u, 0}\left(p-\left[u^{\prime}\right]\right) \leq t / p$. In other words, condition (d) is symmetric in $u$ and $u^{\prime}$.

This means that to prove that $(\mathrm{d}) \Longrightarrow(\mathrm{a})$, it is sufficient to check that $(\mathrm{d}) \Longrightarrow(\mathrm{b})$. Given $(\mathrm{d})$, for $x \in W(\mathfrak{o})$, apply Lemma 5.7 to construct a stable presentation $x_{0}, x_{1}, \ldots$ of $x$ with respect to $u$. By Theorem $5.11(\mathrm{~b}), \tilde{\beta}_{u, t}(x)=$ $\max _{i}\left\{(t / p)^{i} \lambda(\tilde{\alpha})\left(x_{i}\right)\right\}$. Applying $\tilde{\beta}_{u^{\prime}, 0}$ to the identity $x=\sum_{i} x_{i}(p-[u])^{i}$ then gives $\tilde{\beta}_{u, t}(x) \geq \tilde{\beta}_{u^{\prime}, 0}(x)$. Lemma 7.10 and Remark 7.7 then give

$$
\tilde{\beta}_{u, t}=H\left(\tilde{\beta}_{u, t}, t\right) \geq H\left(\tilde{\beta}_{u^{\prime}, 0}, t\right)=\tilde{\beta}_{u^{\prime}, t},
$$

yielding (b) and completing the proof.

Lemma 8.3 allows us to replace the center $u$ of the norm $\tilde{\beta}_{u, t}$ with a nearby value, as was critical in the analysis of $\mathcal{M}(K[T])$.

Corollary 8.4. For $u, u^{\prime} \in \tilde{\mathfrak{o}}$ with $\tilde{\alpha}(u), \tilde{\alpha}\left(u^{\prime}\right) \leq p^{-1}$ and $t \in(0,1]$, if $\lambda(\alpha)\left([u]-\left[u^{\prime}\right]\right) \leq t / p$, then $\tilde{\beta}_{u, t}=\tilde{\beta}_{u^{\prime}, t}$.

Proof. Since $\tilde{\beta}_{u, t} \leq \lambda(\alpha)$, this follows from Lemma 8.3.

Corollary 8.5. For $u \in \tilde{\mathfrak{o}}$ with $\tilde{\alpha}(u) \leq p^{-1}$ and $t \in(0,1]$, there exists $u^{\prime} \in \tilde{\mathfrak{o}}$ which is integral over o such that $\tilde{\alpha}\left(u^{\prime}\right) \leq p^{-1}, \lambda(\tilde{\alpha})\left([u]-\left[u^{\prime}\right]\right)<t / p$, and $\tilde{\beta}_{u, t}=\tilde{\beta}_{u^{\prime}, t}$.

Proof. By Remark 3.7, $[u]-\left[u^{\prime}\right]=\sum_{i=0}^{\infty} p^{i}\left[P_{i}\right]$ for some polynomials $P_{i}$ in $u^{p^{-i}},\left(u^{\prime}\right)^{p^{-i}}$ such that $P_{i}$ is homogeneous of degree $p^{i}$ and divisible by $u^{p^{-i}}-\left(u^{\prime}\right)^{p^{-i}}$. It follows that

$$
\lambda(\tilde{\alpha})\left([u]-\left[u^{\prime}\right]\right) \leq \max _{i}\left\{p^{-i} \tilde{\alpha}\left(u-u^{\prime}\right)^{p^{-i}}\right\}
$$

We can make the right-hand side smaller than $t / p$ by ensuring that $\tilde{\alpha}(u-$ $\left.u^{\prime}\right)<\left(t p^{i-1}\right)^{p^{i}}$ for each of the finitely many nonnegative integers $i$ for which $p^{-i} \geq t / p$; this is possible because the integral closure of $\mathfrak{o}$ in $\tilde{\mathfrak{o}}$ is dense. By Corollary 8.4, we obtain the desired result.

REMARK 8.6. Define the function $d\left(u, u^{\prime}\right)=p \tilde{\beta}_{u^{\prime}, 0}(p-[u])$. If $d\left(u, u^{\prime}\right)$, $d\left(u^{\prime}, u^{\prime \prime}\right) \leq t$, then Lemma 8.3 gives $\tilde{\beta}_{u^{\prime}, t}=\tilde{\beta}_{u, t}=\tilde{\beta}_{u^{\prime \prime}, t}$, and hence $d\left(u, u^{\prime \prime}\right) \leq t$. In other words, the function $d$ satisfies the strong triangle inequality $d\left(u, u^{\prime \prime}\right) \leq \max \left\{d\left(u, u^{\prime}\right), d\left(u^{\prime}, u^{\prime \prime}\right)\right\}$. Lemma 8.3 also implies the symmetry property $d\left(u, u^{\prime}\right)=d\left(u^{\prime}, u\right)$. This almost implies that $d$ is an ultrametric distance function, but not quite: we can have $d\left(u, u^{\prime}\right)=0$ even when $u \neq u^{\prime}$. 
(That is, $d$ is a pseudometric rather than a true metric.) Nonetheless, the function $d$ will play a role in the following arguments similar to that played by the usual distance function on $K$ in the analysis of $\mathcal{M}(K[T])$.

We can now give an analogue of Lemma 2.17.

Lemma 8.7. For $u \in \tilde{\mathfrak{o}}$ with $\tilde{\alpha}(u) \leq p^{-1}$ and $s, t \in[0,1], \beta_{u, s} \geq \beta_{u, t}$ if and only if $s \geq t$.

Proof. If $s \geq t$, then evidently $\beta_{u, s} \geq \beta_{u, t}$. It remains to show that if $s>t$, then $\beta_{u, s} \neq \beta_{u, t}$; it is enough to check this when $t>0$. By Corollary 8.5, we can choose $u^{\prime} \in \tilde{\mathfrak{o}}$ integral over $\mathfrak{o}$ with $\tilde{\alpha}\left(u^{\prime}\right) \leq p^{-1}$ for which $\beta_{u, t}=\beta_{u^{\prime}, t}$, and hence $\beta_{u, s}=\beta_{u^{\prime}, s}$ by Lemma 7.10. Let $P(T)=\prod_{i=1}^{m}\left(T-u_{i}\right)$ be the minimal polynomial of $u^{\prime}$ over $\mathfrak{o}$. Then $\tilde{\beta}_{u^{\prime}, s}\left(p-\left[u_{i}\right]\right) \geq \tilde{\beta}_{u^{\prime}, t}\left(p-\left[u_{i}\right]\right)$ with strict inequality when $u_{i}=u^{\prime}$. If we put $y=\prod_{i=1}^{m}\left(p-\left[u_{i}\right]\right) \in W(\mathfrak{o})$, then $\beta_{u, s}(y)=\tilde{\beta}_{u^{\prime}, s}(y)>\tilde{\beta}_{u^{\prime}, t}(y)=\beta_{u, t}(y)$, so $\beta_{u, s} \neq \beta_{u, t}$, as desired.

Corollary 8.8. For $u \in \tilde{\mathfrak{o}}$ with $\tilde{\alpha}(u) \leq p^{-1}$ and $t \in[0,1], r\left(\beta_{u, t}\right)=t$.

Proof. This follows from Lemma 8.7 plus Lemma 7.10.

We also have an analogue of Lemma 2.19.

LEMMA 8.9. For $u, u^{\prime} \in \tilde{\mathfrak{o}}$ with $\tilde{\alpha}(u), \tilde{\alpha}\left(u^{\prime}\right) \leq p^{-1}$ and $t \in[0,1]$, the following are equivalent.

(a) We have $\beta_{u, t}=\beta_{u^{\prime}, t}$.

(b) We have $\beta_{u, t} \geq \beta_{u^{\prime}, t}$.

(c) We have $\beta_{u, t} \geq \beta_{u^{\prime}, 0}$.

(d) There exists $\tau \in \operatorname{Aut}(\tilde{\mathfrak{o}} / \mathfrak{o})$ for which $t / p \geq \tilde{\beta}_{u^{\prime}, 0}(p-[\tau(u)])$.

Proof. Assume first that $t>0$. By Lemma 8.3, we have (d) $\Longrightarrow(\mathrm{a}) \Longrightarrow$ (b) $\Longrightarrow(\mathrm{c})$, so it remains to check that $(\mathrm{c}) \Longrightarrow(\mathrm{d})$. Assume (c); then apply Corollary 8.5 to construct $v \in \tilde{\mathfrak{o}}$ integral over $\mathfrak{o}$ with $\tilde{\alpha}^{-1}(v)=p^{-1}$ for which $\lambda(\tilde{\alpha})([u]-[v])<t / p$ and $\tilde{\beta}_{u, t}=\tilde{\beta}_{v, t}$. Let $P(T)=\prod_{i=1}^{m}\left(T-v_{i}\right)$ be the minimal polynomial of $v$ over $\mathfrak{o}$, with the roots ordered so that the sequence $t_{i}=$ $p \tilde{\beta}_{u^{\prime}, 0}\left(p-\left[v_{i}\right]\right)$ is nondecreasing.

If (d) fails, then also $t / p<\tilde{\beta}_{u^{\prime}, 0}(p-[\tau(v)])$, so $t_{i}>t$ for $i=1, \ldots, m$. We exploit transitivity as in Remark 8.6: since $t_{i} \geq t_{1}$, by Lemma 8.3 we have $\tilde{\beta}_{u^{\prime}, t_{i}}=\tilde{\beta}_{v_{i}, t_{i}}$ and $\tilde{\beta}_{u^{\prime}, t_{i}}=\tilde{\beta}_{v_{1}, t_{i}}$, so $\tilde{\beta}_{v_{i}, t_{i}}=\tilde{\beta}_{v_{1}, t_{i}}$. By Lemma 8.3 again,

$$
\max \left\{t / p, \tilde{\beta}_{v_{1}, 0}\left(p-\left[v_{i}\right]\right)\right\} \leq t_{i} / p .
$$

This inequality becomes strict for $i=1$. 
If we put $y=\prod_{i=1}^{m}\left(p-\left[v_{i}\right]\right) \in W(\mathfrak{o})$, then by Lemma 5.13 and (8.9.1),

$$
\begin{aligned}
\beta_{u, t}(y) & =\tilde{\beta}_{v_{1}, t}(y)=\prod_{i=1}^{m} \tilde{\beta}_{v_{1}, t}\left(p-\left[v_{i}\right]\right) \\
& =\prod_{i=1}^{m} \max \left\{t / p, \tilde{\beta}_{v_{1}, 0}\left(p-\left[v_{i}\right]\right)\right\} \\
& <\prod_{i=1}^{m}\left(t_{i} / p\right)=\prod_{i=1}^{m} \tilde{\beta}_{u^{\prime}, 0}\left(p-\left[v_{i}\right]\right)=\beta_{u^{\prime}, 0}(y)
\end{aligned}
$$

a contradiction. Hence, (d) holds, as desired.

Suppose now that $t=0$. Note that each condition for $t=0$ implies the corresponding condition for all $t>0$. For (a)-(c), the converse implication is clear; the converse implication also holds for (d) by the completeness of $\tilde{\mathfrak{o}}$ and the compactness of $\operatorname{Aut}(\tilde{\mathfrak{o}} / \mathfrak{o})$. We may thus reduce the claim to the case $t>0$ treated above.

We are now ready to make the decisive step, analogous to Lemma 2.20.

Lemma 8.10. For $\beta \in \mu^{-1}(\alpha)$ and $s \in(r(\beta), 1]$, there exists $u \in \mathfrak{o}$ with $\tilde{\alpha}(u) \leq p^{-1}$ for which $H(\beta, s)=\beta_{u, s}$.

Proof. Let $S$ be the set of $s \in[0,1]$ for which $\beta_{u, s} \geq \beta$ for some $u \in \tilde{\mathfrak{o}}$ with $\tilde{\alpha}(u) \leq p^{-1}$. The set $S$ is up-closed and nonempty; let $t$ be its infimum. As in the proof of Lemma 2.20, it suffices to check that $r(\beta) \geq t$.

By proceeding as in Definition 7.5, we can construct an isometric embedding $\mathfrak{o} \rightarrow \mathfrak{o}_{1}$ of complete valuation rings of characteristic $p$, with the norm on $\mathfrak{o}_{1}$ denoted by $\alpha_{1}$, and an element $v \in \mathfrak{o}_{1}$ with $\alpha_{1}(v)=p^{-1}$, for which $\beta$ is the restriction of the seminorm $H\left(\alpha_{1}, v, 0\right)$. There is no harm in further enlarging $\mathfrak{o}_{1}$ so that Frac $\mathfrak{o}_{1}$ becomes algebraically closed; we may then identify $\tilde{\mathfrak{o}}$ with a subring of $\mathfrak{o}_{1}$.

For $u \in \mathfrak{o}$, if $\tilde{\alpha}(u)<p^{-1}$, then by Lemma $5.13, H\left(\alpha_{1}, v, s\right)(p-[u])$ is constant on $[0,1]$. If instead $\tilde{\alpha}(u)=p^{-1}$, then for $s \in[0, t)$ we have $H(\beta, s) \neq$ $\beta_{u, s}$, so by Lemma $8.9, s / p<H\left(\alpha_{1}, v, 0\right)(p-[u])$. By Lemma 5.13, for $s \in$ $[0, t]$

$\left.H\left(\alpha_{1}, v, s\right)(p-[u])=\max \left\{s / p, H\left(\alpha_{1}, v, 0\right)(p-[u])\right\}=H\left(\alpha_{1}, v, 0\right)(p-[u])\right\}$.

For each nonzero $x \in W(\mathfrak{o})$, by Theorem 6.8 we have $x=y\left(p-\left[u_{1}\right]\right) \cdots(p-$ $\left.\left[u_{n}\right]\right)$ for some stable $y \in W(\tilde{\mathfrak{o}})$ and some $u_{1}, \ldots, u_{n} \in \tilde{\mathfrak{o}}$ with $\tilde{\alpha}\left(u_{i}\right) \leq p^{-1}$. 
For $s \in[0, t]$,

$$
H(\beta, s)(x)=H\left(\alpha_{1}, v, s\right)(x)=\lambda(\tilde{\alpha})(y) \prod_{i=1}^{n} H\left(\alpha_{1}, v, 0\right)\left(p-\left[u_{i}\right]\right)
$$

is independent of $s$. Hence, $H(\beta, s)=\beta$ for $s \in[0, t]$, and so $r(\beta) \geq t$, as desired.

Corollary 8.11. Suppose that $\beta \in \mu^{-1}(\alpha)$ is such that $\beta \neq \beta_{u, t}$ for all $u \in \tilde{\mathfrak{o}}$ with $\tilde{\alpha}(u) \leq p^{-1}$ and all $t \in[0,1]$. Then for each $y \in W(\mathfrak{o})$, for any sufficiently small $s \in(r(\beta), 1], \beta(y)=H(\beta, s)(y)$.

With this analysis, we obtain Theorem 7.12 as follows.

Lemma 8.12. Theorem 7.12 holds in case $R=\mathfrak{o}$.

Proof. If $r(\beta)=1$, then $\beta=H(\beta, 1)=H(\gamma, 1)$ by Theorem 7.8(b). If $r(\gamma)=1$, then by Theorem 7.8 (b) again, $\beta \geq \gamma=H(\gamma, 1)=H(\beta, 1) \geq \beta$, and so $\beta=H(\gamma, 1)$. It is thus safe to assume that $r(\beta), r(\gamma)<1$.

For each $s \in(\max \{r(\beta), r(\gamma)\}, 1]$, by Lemma 8.10 we have $H(\beta, s)=$ $\beta_{u, s}, H(\gamma, s)=\beta_{u^{\prime}, s}$ for some $u, u^{\prime} \in \tilde{\mathfrak{o}}$ with $\tilde{\alpha}(u), \tilde{\alpha}\left(u^{\prime}\right) \leq p^{-1}$. Since $\beta \geq \gamma$ implies that $H(\beta, s) \geq H(\gamma, s)$ by Remark 7.7, we have $\beta_{u, s} \geq \beta_{u^{\prime}, s}$, but by Lemma 8.9, this forces $\beta_{u, s}=\beta_{u^{\prime}, s}$. Hence, $H(\beta, s)=H(\gamma, s)$.

If $r(\gamma)>r(\beta)$, by taking the limit as $s \rightarrow r(\gamma)^{+}$, we deduce that $\gamma=$ $H(\beta, r(\gamma))=H(\beta, r(\beta))=\beta$, a contradiction. Hence, $r(\beta) \geq r(\gamma)$, and by taking the limit as $s \rightarrow r(\beta)^{+}$, we deduce that $\beta=H(\gamma, r(\beta))$, as desired.

We derive the following corollary analogous to Corollary 2.23.

Corollary 8.13. For any $\beta, \gamma \in \mu^{-1}(\alpha)$ with $\beta \geq \gamma$, there exist $\tilde{\beta}, \tilde{\gamma} \in$ $\mu^{-1}(\tilde{\alpha})$ restricting to $\beta, \gamma$, respectively, for which $\tilde{\beta} \geq \tilde{\gamma}$.

Proof. Extend $\gamma$ as in the proof of Theorem $7.8(\mathrm{~d})$; then put $\tilde{\beta}=$ $H(\tilde{\gamma}, r(\beta))$. This restricts to $\beta$ by Theorem 7.12 .

To obtain an analogue of Corollary 2.25, we must make the function $d\left(u, u^{\prime}\right)$ from Remark 8.6 more explicit.

Lemma 8.14. Consider $u, u^{\prime} \in \tilde{\mathfrak{o}}$ with $\tilde{\alpha}(u), \tilde{\alpha}\left(u^{\prime}\right) \leq p^{-1}$.

(a) If $u=0$ or $\tilde{\alpha}\left(u^{\prime}-u\right)>p^{-p /(p-1)} \max \left\{\tilde{\alpha}(u), \tilde{\alpha}\left(u^{\prime}\right)\right\}$, then $d\left(u, u^{\prime}\right)=$ $p \tilde{\alpha}\left(u^{\prime}-u\right)$. 
(b) If $u \neq 0$ and there exists a nonnegative integer $i$ for which $\tilde{\alpha}\left(u^{\prime} / u-1\right) \in$ $\left(p^{-p^{i+1} /(p-1)}, p^{-p^{i} /(p-1)}\right)$, then

$$
d\left(u, u^{\prime}\right)=p^{1-i} \tilde{\alpha}(u) \tilde{\alpha}\left(u^{\prime} / u-1\right)^{p^{-i}} \in\left(p^{-i-1 /(p-1)} \tilde{\alpha}(u), p^{-(i-1)-1 /(p-1)} \tilde{\alpha}(u)\right) .
$$

(c) If $u \neq 0$ and there exists a positive integer $i$ for which $\tilde{\alpha}\left(u^{\prime} / u-1\right)=$ $p^{-p^{i} /(p-1)}$, then $d\left(u, u^{\prime}\right) \leq p^{-(i-1)-1 /(p-1)} \tilde{\alpha}(u)$, with equality unless $\tilde{\alpha}(u)=p^{-1}$ and $\tilde{\alpha}\left(1-u^{p^{i}}\left(u^{\prime} / u-1\right)^{1-p}\right)<1$.

Proof. Part (a) is clear when $u=0$, so we may assume that $u \neq 0$ throughout. Write

$$
\left[u^{\prime} / u\right]-1=\sum_{j=0}^{\infty} p^{j}\left[P_{j}\left(\left(u^{\prime} / u-1\right)^{p^{-j}}\right)\right]
$$

for $P_{j}(T) \in \mathbb{F}_{p}[T]$ as in Lemma 3.8. By Lemma 3.8, $P_{j}(T)$ is divisible by $T$ but not by $T^{2}$; consequently, if $\tilde{\alpha}\left(u^{\prime} / u-1\right)<1$, then

$$
\tilde{\alpha}\left(P_{j}\left(\left(u^{\prime} / u-1\right)^{p^{-j}}\right)\right)=\tilde{\alpha}\left(u^{\prime} / u-1\right)^{p^{-j}},
$$

and so

$$
\lambda(\tilde{\alpha})\left(\left[u^{\prime}\right]-[u]\right)=\max _{j}\left\{p^{-j} \tilde{\alpha}(u) \tilde{\alpha}\left(u^{\prime} / u-1\right)^{p^{-j}}\right\} .
$$

In case (a), the maximum in (8.14.2) is achieved only by the index $j=0$; in case (b), the maximum is achieved only by $j=i$. In these cases, the righthand side of (8.14.1) is dominated under $\lambda(\tilde{\alpha})$ by a single term which is a power of $p$ times a Teichmüller element, so this term also dominates under $\tilde{\beta}_{u, 0}$. This yields the desired results in these cases.

In case (c), the maximum in (8.14.2) is achieved only by the indices $j=$ $i-1, i$. We modify the presentation of $\left[u^{\prime} / u\right]-1$ by replacing $p^{i}\left[P_{i}\left(\left(u^{\prime} / u-\right.\right.\right.$ $\left.\left.1)^{p^{-i}}\right)\right]$ with $p^{i-1}\left[u P_{i}\left(\left(u^{\prime} / u-1\right)^{p^{-i}}\right)\right]$. We then observe that $d\left(u, u^{\prime}\right) \leq$ $p^{-(i-1)-1 /(p-1)} \tilde{\alpha}(u)$ with equality unless

$$
\tilde{\alpha}\left(\left(u^{\prime} / u-1\right)^{p^{-i}}-u\left(u^{\prime} / u-1\right)^{p^{-i+1}}\right)<p^{-1 /(p-1)},
$$

which yields the desired result.

Corollary 8.15. For $u \in \tilde{\mathfrak{o}}$ with $\tilde{\alpha}(u) \leq p^{-1}$ and $0<s<t \leq 1$, there are infinitely many points of $\mu^{-1}(\tilde{\alpha})$ of the form $\tilde{\beta}_{u^{\prime}, s}$ which are dominated by $\tilde{\beta}_{u, t}$. 
Proof. Suppose first that $u=0$; then for any $u^{\prime} \in \tilde{\mathfrak{o}}$, we have $\tilde{\beta}_{u, 0}(p-$ $\left.\left[u^{\prime}\right]\right)=\tilde{\alpha}\left(u^{\prime}\right)$ because $\tilde{\beta}_{u, 0}(p)=0$. Choose $s^{\prime} \in(s, t)$ for which $s^{\prime} / p$ occurs as the norm of some element of $\tilde{\mathfrak{o}}$. As in the proof of Lemma 2.24, we can find an infinite subset $S$ of $\tilde{\mathfrak{o}}$ such that $\tilde{\alpha}\left(u^{\prime}\right)=\tilde{\alpha}\left(u^{\prime}-u^{\prime \prime}\right)=s^{\prime} / p$ for all distinct $u^{\prime}, u^{\prime \prime} \in S$. We then have $\tilde{\beta}_{u, t} \geq \tilde{\beta}_{u^{\prime}, s}$ for all $u^{\prime} \in S$ by Lemma 8.3. Moreover, for $u^{\prime}, u^{\prime \prime} \in S$ distinct, $\left[u^{\prime}\right]-\left[u^{\prime \prime}\right]$ is stable, so $\tilde{\beta}_{u^{\prime}, 0}\left(p-\left[u^{\prime \prime}\right]\right)=$ $\tilde{\beta}_{u^{\prime}, 0}\left(\left[u^{\prime}\right]-\left[u^{\prime \prime}\right]\right)=s^{\prime} / p$, and hence $\tilde{\beta}_{u^{\prime}, s} \neq \tilde{\beta}_{u^{\prime \prime}, s}$ by Lemma 8.3 again.

Suppose next that $u \neq 0$. Choose $c \in\left(p^{-p /(p-1)}, p^{-1 /(p-1)}\right)$ occurring as the norm of an element of $\tilde{\mathfrak{o}}$ and such that $p^{-i+1} \tilde{\alpha}(u) c \in(s, t)$ for some nonnegative integer $i$. Again as in Lemma 2.24, we choose an infinite subset $S$ of $\tilde{\mathfrak{o}}$ such that $\tilde{\alpha}\left(u^{\prime} / u-1\right)=\tilde{\alpha}\left(u^{\prime} / u-u^{\prime \prime} / u\right)=c^{p^{i}}$ for all distinct $u^{\prime}, u^{\prime \prime} \in S$. By Lemma 8.14, we have $\tilde{\beta}_{u, 0}\left(p-\left[u^{\prime}\right]\right)=\tilde{\beta}_{u^{\prime}, 0}\left(p-\left[u^{\prime \prime}\right]\right)=p^{-i} \tilde{\alpha}(u) c$ for all distinct $u^{\prime}, u^{\prime \prime} \in S$. By Lemma 8.3, $\tilde{\beta}_{u, t} \geq \tilde{\beta}_{u^{\prime}, s}$ for all $u^{\prime} \in S$, and $\tilde{\beta}_{u^{\prime}, s} \neq \tilde{\beta}_{u^{\prime \prime}, s}$ for all distinct $u^{\prime}, u^{\prime \prime} \in S$.

We can now derive an analogue of Lemma 2.24.

Lemma 8.16. For $u \in \tilde{\mathfrak{o}}$ with $\tilde{\alpha}(u) \leq p^{-1}$ and $t \in[0,1]$, let $D(u, t)$ be the set of $\beta_{v, 0} \in \mu^{-1}(\alpha)$ dominated by $\beta_{u, t}$. Then for $s, t \in[0,1], D(u, s)=$ $D(u, t)$ if and only if $s=t$.

Proof. It suffices to deduce a contradiction under the assumption that $D(u, s)=D(u, t)$ for some $t>s>0$. By Corollary 8.5, we can find $u^{\prime} \in \tilde{\mathfrak{o}}$ integral over $\mathfrak{o}$ for which $\tilde{\beta}_{u, s}=\tilde{\beta}_{u^{\prime}, s}$, so that $D(u, t)=D\left(u^{\prime}, t\right)$ and $D(u, s)=$ $D\left(u^{\prime}, s\right)$. Since $D(u, t)=D(u, s)$, for any $\beta_{v, 0} \in D(u, t)$, we have $\beta_{u^{\prime}, s} \geq \beta_{v, 0}$, and hence (by Lemma 8.9) $\tilde{\beta}_{v, 0}\left(p-\left[\tau\left(u^{\prime}\right)\right]\right) \leq s / p$ for some $\tau \in \operatorname{Aut}(\tilde{\mathfrak{o}} / \mathfrak{o})$. Consequently, there are only finitely many points in $\mu^{-1}(\tilde{\alpha})$ of the form $\tilde{\beta}_{v, s}$ which are dominated by $\tilde{\beta}_{u, t}$; however, this would contradict Corollary 8.15. This contradiction establishes the desired result.

We also derive the following analogue of Theorem 2.26.

THEOREM 8.17. Each element of $\mu^{-1}(\alpha)$ is of exactly one of the following four types.

(i) A point of the form $\beta_{u, 0}$ for some $u \in \tilde{\mathfrak{o}}$ with $\tilde{\alpha}(u) \leq p^{-1}$. Such a point has radius 0 and is minimal.

(ii) A point of the form $\beta_{u, t}$ for some $u \in \tilde{\mathfrak{o}}$ with $\tilde{\alpha}(u) \leq p^{-1}$ and some $t \in(0,1)$ such that $t / p$ is the norm of an element of $\tilde{\mathfrak{o}}$. Such a point has radius $t$ and is not minimal. 
(iii) A point of the form $\beta_{u, t}$ for some $u \in \tilde{\mathfrak{o}}$ with $\tilde{\alpha}(u) \leq p^{-1}$ and some $t \in(0,1)$ such that $t / p$ is not the norm of an element of $\tilde{\mathfrak{o}}$. Such a point has radius $t$ and is not minimal.

(iv) The infimum of a sequence $\beta_{u_{i}, t_{i}}$ for which the sequence $D\left(u_{i}, t_{i}\right)$ is decreasing with empty intersection. Such a point has radius $\inf _{i}\left\{t_{i}\right\}>0$ and is minimal.

Proof. By Corollary 8.8, $r\left(\beta_{u, t}\right)=t$. Consequently, types (i)-(iii) are mutually exclusive. Moreover, $\beta_{u, t}$ cannot be of type (iv), because otherwise $\beta_{u, 0}$ would belong to the empty intersection $\bigcap_{i} D\left(u_{i}, t_{i}\right)$. Consequently, no point can be of more than one type.

It remains to check that any point $\beta \in \mu^{-1}(\alpha)$ not of the form $\beta_{u, t}$ is of type (iv) and is minimal of the claimed radius. Choose a sequence $1 \geq t_{1}>t_{2}>\cdots$ with infimum $r(\beta)$. By Lemma 8.10, for each $i$, we have $H\left(\beta, t_{i}\right)=\beta_{u_{i}, t_{i}}$ for some $u_{i} \in \tilde{\mathfrak{o}}$. The sequence $\beta_{u_{1}, t_{1}}, \beta_{u_{2}, t_{2}}, \ldots$ is decreasing with infimum $\beta$; the sequence $D\left(u_{i}, t_{i}\right)$ is also decreasing. For each $u \in \tilde{\mathfrak{o}}$, there exists $i$ for which for which $\beta_{u, t_{i}} \neq \beta_{u_{i}, t_{i}}$; for such $i$ we have $\beta_{u, 0} \notin D\left(u_{i}, t_{i}\right)$ by Lemma 8.9. Hence, the $D\left(u_{i}, t_{i}\right)$ have empty intersection. Hence, $\beta$ is of type (iv); it is minimal by Theorem 7.12 plus Lemma 8.10. Since $\beta=\inf _{i}\left\{\beta_{u_{i}, t_{i}}\right\}$ and $r\left(\beta_{u_{i}, t_{i}}\right)=t_{i}$ by Corollary 8.8, we have $r(\beta) \geq$ $\inf _{i}\left\{t_{i}\right\}$; the reverse inequality also holds because $t_{i}=r\left(\beta_{u_{i}, t_{i}}\right) \geq r(\beta)$ by Theorem 7.12 .

Suppose by way of contradiction that $r(\beta)=0$. By Corollary 8.5, we may choose the $u_{i}$ to be integral over $\mathfrak{o}$. Let $U_{0}$ denote the original sequence $u_{1}, u_{2}, \ldots$ For $h=1,2, \ldots$, we construct a subsequence $U_{h}$ of $U_{h-1}$ such that any two terms $v_{1}, v_{2}$ of $U_{h}$ satisfy $d\left(v_{1}, v_{2}\right) \leq p^{-h}$, as follows. Given $U_{h-1}$, for $i$ sufficiently large, whenever $u_{i} \in U_{h-1}$, we have $t_{i} \leq p^{-h}$ and $\beta_{u_{i}, t_{i}}=\beta_{u_{j}, t_{i}}$ for all $j \geq i$ with $u_{j} \in U_{h-1}$. By Lemma 8.7 and the integrality of $u_{i}$ over $\mathfrak{o}$, this limits the $u_{j}$ to finitely many closed disks of radius $t_{i}$ under $d$. One of these disks then contains infinitely many elements of $U_{h-1}$; choose these to form the subsequence $U_{h}$.

By diagonalizing (i.e., choosing a subsequence of $U_{0}$ whose $i$ th term belongs to $U_{i}$ for each $i$ ), we obtain a Cauchy sequence in $\tilde{\mathfrak{o}}$ with respect to $d$. By Lemma 8.18 below, this sequence admits a limit $u$ with respect to $d$, which then satisfies $\beta=\beta_{u, 0}$, a contradiction. We conclude that $r(\beta)>0$, as desired. 
LEMMA 8.18. The pseudometric $d$ on the set $\left\{u \in \tilde{\mathfrak{o}}: \tilde{\alpha}(u) \leq p^{-1}\right\}$ is complete. That is, for every sequence $u_{0}, u_{1}, \ldots$ with $\lim _{i, j \rightarrow \infty} d\left(u_{i}, u_{j}\right)=0$, there exists $u$ for which $\lim _{i \rightarrow \infty} d\left(u, u_{i}\right)=0$.

Proof. Let $U_{0}$ denote the original sequence. For $h=0,1, \ldots$, we produce an infinite subsequence $U_{h+1}$ of $U_{h}$ such that any two elements $v_{1}, v_{2}$ of $U_{h+1}$ satisfy $\tilde{\alpha}\left(v_{1}-v_{2}\right) \leq p^{-p^{h+1} /(p-1)}$. To produce $U_{1}$, apply Lemma 8.14 (a). Given $U_{h}$ for some $h>0$, by Lemma 8.14(c), $U_{h}$ falls into $p$ residue classes modulo elements of $\tilde{\mathfrak{o}}$ of norm less than $p^{-p^{h} /(p-1)}$. In particular, one of these residue classes contains infinitely many terms of $U_{h}$; by Lemma 8.14 (b), all but finitely many such terms are pairwise congruent modulo elements of $\tilde{\mathfrak{o}}$ of norm at most $p^{-p^{h+1} /(p-1)}$. We can thus choose these to constitute $U_{h+1}$.

By diagonalizing, we obtain a Cauchy sequence in $\tilde{\mathfrak{o}}$ with respect to $\tilde{\alpha}$, which then has a limit $u$. With respect to $d$, the original sequence is Cauchy, and $u$ is a limit of a subsequence, so it is also a limit of the entire sequence.

In the manner of Corollary 2.27, we can describe the residual extensions and norm groups of points in $\mu^{-1}(\alpha)$.

COROllary 8.19. Let $\beta$ be a point of $\mu^{-1}(\alpha)$, classified according to Theorem 8.17. Let $\left|\alpha^{\times}\right|,\left|\beta^{\times}\right|$denote the groups of nonzero values assumed by $\alpha, \beta$, respectively. Put $K=\operatorname{Frac}(\mathfrak{o})$.

(i) For $\beta$ of type $(i), \kappa_{\mathcal{H}(\beta)}$ is algebraic over $\kappa_{K}$, and $\left|\beta^{\times}\right| /\left|\alpha^{\times}\right|$is a torsion group.

(ii) For $\beta$ of type (ii), $\kappa_{\mathcal{H}(\beta)}$ is finitely generated over $\kappa_{K}$ of transcendence degree 1 , and $\left|\beta^{\times}\right| /\left|\alpha^{\times}\right|$is a finite group.

(iii) For $\beta$ of type (iii), $\kappa_{\mathcal{H}(\beta)}$ is a finite extension of $\kappa_{K}$, and $\left|\beta^{\times}\right| /\left|\alpha^{\times}\right|$is a finitely generated abelian group of rank 1 .

(iv) For $\beta$ of type (iv), $\kappa_{\mathcal{H}(\beta)}$ is algebraic over $\kappa_{K}$, and $\left|\beta^{\times}\right| /\left|\alpha^{\times}\right|$is a torsion group.

Proof. By Ostrowski's theorem again (see (2.27.1)), in cases (ii) and (iii), it is enough to check the claims after replacing $K$ by a finite extension; in cases (i) and (iv), we may replace $K$ by a completed algebraic closure. We make these assumptions hereafter.

In cases (i)-(iii), we have $\beta=\beta_{u, t}$ with $u \in \mathfrak{o}$ and $\alpha(u) \leq p^{-1}$. For each $x \in$ $W(\mathfrak{o})$, by Lemma 5.7 , in $W(\tilde{\mathfrak{o}})$ there exists a stable presentation $x_{0}, x_{1}, \ldots$ of $x$ with respect to $u$. Let $\overline{x_{i}} \in \tilde{\mathfrak{o}}$ denote the reduction modulo $p$ of $x_{i}$. By 
Theorem 5.11(b),

$$
\beta_{u, t}(x)=\max _{i}\left\{(t / p)^{i} \tilde{\alpha}\left(\overline{x_{i}}\right)\right\}
$$

Consequently, in cases (i) and (ii), $\left|\beta^{\times}\right| /\left|\alpha^{\times}\right|$is trivial; in case (iii), $\left|\beta^{\times}\right| /\left|\alpha^{\times}\right|$ is freely generated by $t / p$.

In case $(\mathrm{i}), \kappa_{\mathcal{H}(\beta)}$ may be identified with the quotient of $W(\mathfrak{o})$ by the ideal $(p,[u])$, so $\kappa_{\mathcal{H}(\beta)}=\kappa_{K}$. In case (ii), pick $v \in \mathfrak{o}$ with $\alpha(v)=t / p$. For $R=W(\mathfrak{o})\left[[v]^{-1}\right]$, we have $\mathfrak{o}_{R} / \mathfrak{m}_{R} \cong \kappa_{K}[z]$ for $z$ the class of $[v]^{-1}(p-[u])$. Consequently, $\kappa_{\mathcal{H}(\beta)}=\kappa_{K}(z)$. In case (iii), if $x \neq 0$, then the maximum in (8.19.1) is achieved only by a single index $i$. For this $i$, we have $\beta_{u, t}(x-(p-$ $\left.[u])^{i}\left[\overline{x_{i}}\right]\right)<\beta_{u, t}(x)$; it follows that each element of $\kappa_{\mathcal{H}(\beta)}$ is represented by a Teichmüller element. Consequently, $\kappa_{\mathcal{H}(\beta)}=\kappa_{K}$.

In case (iv), by Corollary 8.11, for each $y \in W(\mathfrak{o})$, any sufficiently small $s \in(r(\beta), 1]$ satisfies $H(\beta, s)(y)=\beta(y)$. If we choose $s \in\left|\alpha^{\times}\right|$, we deduce that $\left|\beta^{\times}\right| /\left|\alpha^{\times}\right|$is trivial. If we choose $s \notin\left|\alpha^{\times}\right|$, then for any $z \in W(\mathfrak{o})$ with $\beta(z) \leq \beta(y)$, by case (iii), there must exist $\lambda \in \mathfrak{o}$ for which $H(\beta, s)(z-[\lambda] y)<$ $H(\beta, s)(y)$. This implies that

$$
\beta(z-[\lambda] y) \leq H(\beta, s)(z-[\lambda] y)<H(\beta, s)(y)=\beta(y),
$$

so $z / y$ and $[\lambda]$ have the same image in $\kappa_{\mathcal{H}(\beta)}$. Hence, $\kappa_{\mathcal{H}(\beta)}=\kappa_{K}$.

REMARK 8.20. One could also consider points of $\mu^{-1}(\alpha)$ obtained by restricting points of $\mu^{-1}(\tilde{\alpha})$ of the form $H(\tilde{\alpha}, \pi, t)$ for $\pi \in W(\tilde{\mathfrak{o}})$ as in Hypothesis 5.1; that is, $\pi=\sum_{i=0}^{\infty} p^{i}\left[\overline{\pi_{i}}\right]$ with $\tilde{\alpha}\left(\overline{\pi_{0}}\right) \leq p^{-1}$ and $\tilde{\alpha}\left(\overline{\pi_{1}}\right)=1$. However, by Theorem 6.8 , any such $\pi$ generates the same ideal as $p-[u]$ for some $u \in \tilde{\mathfrak{o}}$, so $H(\tilde{\alpha}, \pi, t)=H(\tilde{\alpha}, u, t)$ for $t \in[0,1]$. Consequently, these points are again of types (i)-(iii) in Theorem 8.17 and not of type (iv).

Acknowledgments. The author thanks Laurent Fargues and Jean-Marc Fontaine for providing a preliminary draft of [11], Michael Temkin for helpful discussions, and Peter Scholze and Liang Xiao for feedback on early drafts of this article and particularly concerning Lemma 5.5.

\section{REFERENCES}

[1] M. Baker and R. Rumely, Potential Theory and Dynamics on the Berkovich Projective Line, Math. Surveys Monogr. 159, Amer. Math. Soc., Providence, 2010. MR 2599526. 
[2] V. G. Berkovich, Spectral Theory and Analytic Geometry over Non-Archimedean Fields, Math. Surveys Monogr. 33, Amer. Math. Soc., Providence, 1990. MR 1070709.

[3] - Étale cohomology for non-Archimedean analytic spaces, Publ. Math. Inst. Hautes Études Sci. 78 (1993), 5-161. MR 1259429.

[4] - Smooth p-adic analytic spaces are locally contractible, Invent. Math. 137 (1999), 1-84. MR 1702143. DOI 10.1007/s002220050323.

[5] _ "Smooth $p$-adic analytic spaces are locally contractible, II" in Geometric Aspects of Dwork Theory, de Gruyter, Berlin, 2004, 293-370. MR 2023293.

[6] _ "A non-Archimedean interpretation of the weight zero subspaces of limit mixed Hodge structures" in Algebra, Arithmetic and Geometry, Vol. 1: In Honor of Yu. I. Manin, Progr. Math. 269, Birkhäuser, Boston, 2009, 49-67. MR 2641170. DOI 10.1007/978-0-8176-4745-2_2.

[7] S. Bosch, U. Güntzer, and R. Remmert, Non-Archimedean Analysis: A Systematic Approach to Rigid Analytic Geometry, Grundlehren Math. Wiss. 261, Springer, Berlin, 1984. MR 0746961.

[8] S. Boucksom, C. Favre, and M. Jonsson, Valuations and plurisubharmonic singularities, Publ. Res. Inst. Math. Sci. 44 (2008), 449-494. MR 2426355. DOI 10.2977/ prims/1210167334.

[9] A. Buium, Arithmetic Differential Equations, Math. Surveys Monogr. 118, Amer. Math. Soc., Providence, 2005. MR 2166202.

[10] A. Chambert-Loir, Mesures et équidistribution sur les espaces de Berkovich, J. Reine Angew. Math. 595 (2006), 215-235. MR 2244803. DOI 10.1515/CRELLE.2006.049.

[11] L. Fargues and J.-M. Fontaine, Courbes et fibrés vectoriels en théorie de Hodge $p$-adique, in preparation.

[12] C. Favre and M. Jonsson, The Valuative Tree, Lecture Notes in Math. 1853, Springer, Berlin, 2004. MR 2097722. DOI 10.1007/b100262.

[13] — Valuations and multiplier ideals, J. Amer. Math. Soc. 18 (2005), 655-684. MR 2138140. DOI 10.1090/S0894-0347-05-00481-9.

[14] - Valuative analysis of planar plurisubharmonic functions, Invent. Math. 162 (2005), 271-311. MR 2199007. DOI 10.1007/s00222-005-0443-2.

[15] E. Hrushovski and F. Loeser, Non-archimedean tame topology and stably dominated types, arXiv:1009.0252v2 [math.AG]

[16] A. Joyal, $\delta$-Anneaux et vecteurs de Witt, C. R. Math. Rep. Acad. Sci. Canada 7 (1985), 177-182. MR 0789309.

[17] K. S. Kedlaya, Slope filtrations revisited, Doc. Math. 10 (2005), 447-525. MR 2184462. Correction, Doc. Math. 12 (2007), 361-362. MR 2365906.

[18] - Good formal structures for flat meromorphic connections, I: Surfaces, Duke Math. J. 154 (2010), 343-418. MR 2682186. DOI 10.1215/00127094-2010-041. Correction, Duke Math. J. 161 (2012), 733-734. MR 2891533. DOI 10.1215/ 00127094-1548380.

[19] , p-Adic Differential Equations, Cambridge Stud. Adv. Math. 125, Cambridge University Press, Cambridge, 2010. MR 2663480.

[20] - "Relative $p$-adic Hodge theory and Rapoport-Zink period domains" in Proceedings of the International Congress of Mathematicians (Hyderabad, 2010), Vol. 2, Hindustan Book Agency, New Delhi, 2010, 258-279. MR 2827795.

[21] - Good formal structures for flat meromorphic connections, II: Excellent schemes, J. Amer. Math. Soc. 24 (2011), 183-229. MR 2726603. DOI 10.1090/ S0894-0347-2010-00681-9. 
[22] - Semistable reduction for overconvergent F-isocrystals, IV: Local semistable reduction at nonmonomial valuations, Compos. Math. 147 (2011), 467-523. MR 2776611. DOI 10.1112/S0010437X10005142.

[23] J. Nicaise, Singular cohomology of the analytic Milnor fiber, and mixed Hodge structure on the nearby cohomology, J. Algebraic Geom. 20 (2011), 199-237. MR 2762990. DOI 10.1090/S1056-3911-10-00526-6.

[24] S. Payne, Analytification is the limit of all tropicalizations, Math. Res. Lett. 16 (2009), 543-556. MR 2511632.

[25] P. Ribenboim, The Theory of Classical Valuations, Springer, New York, 1999. MR 1677964.

[26] J.-P. Serre, Local Fields, Grad. Texts in Math. 67, Springer, New York, 1979. MR 0554237.

[27] M. Temkin, Inseparable local uniformization, J. Algebra 373 (2013) 65-119. MR 2995017.

Department of Mathematics

University of California, San Diego

La Jolla, California 92093

$U S A$

kedlaya@ucsd.edu 